\title{
The feasibility of a smoking cessation computer tailored expert system in Dutch general practice and community pharmacy setting
}

Citation for published version (APA):

Hoving, E. F. (2007). The feasibility of a smoking cessation computer tailored expert system in Dutch general practice and community pharmacy setting. [Doctoral Thesis, Maastricht University]. Universiteit Maastricht. https://doi.org/10.26481/dis.20071207eh

Document status and date:

Published: 01/01/2007

DOI:

10.26481/dis.20071207eh

Document Version:

Publisher's PDF, also known as Version of record

Please check the document version of this publication:

- A submitted manuscript is the version of the article upon submission and before peer-review. There can be important differences between the submitted version and the official published version of record.

People interested in the research are advised to contact the author for the final version of the publication, or visit the DOI to the publisher's website.

- The final author version and the galley proof are versions of the publication after peer review.

- The final published version features the final layout of the paper including the volume, issue and page numbers.

Link to publication

\footnotetext{
General rights rights.

- You may freely distribute the URL identifying the publication in the public portal. please follow below link for the End User Agreement:

www.umlib.nl/taverne-license

Take down policy

If you believe that this document breaches copyright please contact us at:

repository@maastrichtuniversity.nl

providing details and we will investigate your claim.
}

Copyright and moral rights for the publications made accessible in the public portal are retained by the authors and/or other copyright owners and it is a condition of accessing publications that users recognise and abide by the legal requirements associated with these

- Users may download and print one copy of any publication from the public portal for the purpose of private study or research.

- You may not further distribute the material or use it for any profit-making activity or commercial gain

If the publication is distributed under the terms of Article $25 \mathrm{fa}$ of the Dutch Copyright Act, indicated by the "Taverne" license above, 


\section{The feasibility of a smoking cessation computer tailored expert system in Dutch general practice and community pharmacy setting}

ter verkrijging van de graad van doctor aan de Universiteit Maastricht, op gezag van de Rector Magnificus, prof. mr. G.P.M.F. Mols volgens het besluit van het College van Decanen, in het openbaar te verdedigen op vrijdag 7 december 2007 om 14.00 uur

door 


\section{Promotor:}

Prof. dr. H. de Vries

\section{Co-promotor:}

Dr. A.N. Mudde (Open Universiteit Nederland)

Beoordelingscommissie:

Prof. dr. H.W. van den Borne (voorzitter)

Prof. dr. L. Lechner (Open Universiteit Nederland)

Dr. I. Mesters

Prof. dr. ir. C.P. van Schayck

Prof. dr. N.K. de Vries

Colofon

Printed by Optima Grafische Communicatie

Lay-out by Ciska Hoving

ISBN: 978-90-8559-161-0

The study presented in this thesis was conducted at the Maastricht Care and Public Health Institute (CAPHRI), which participates in the Netherlands school of Primary Care Research (CaRe), acknowledged in 1995 by the Royal Dutch Academy of Science (KNAW).

The study was conducted with financial support from the Dutch Organisation for Health Research and Development (ZonMw), projectnumber 2200.0002. Publication of this thesis was financially supported by STIVORO for a smokefree future. 


\section{Content}

Chapter 1 General introduction

Chapter 2 Intention to adopt a smoking cessation expert system within a self- 27 selected sample

Chapter 3 Effectiveness of a computer-generated tailored smoking cessation expert system in Dutch community pharmacy and general practice setting

Chapter 4 Determinants of intention to implement a smoking cessation expert system in Dutch general practice

Chapter 5 Pharmacy staff is not ready to incorporate a smoking cessation expert system into their daily routine

Chapter 6 Effect of recruitment method and setting on the composition of 79 samples consisting of adult smokers of Dutch general practitioners

Chapter 7 Smoking and the $\varnothing$ pattern; predictors of transitions through the 97 stages of change

Chapter 8 Predictors of smoking relapse in a sample of Dutch adult smokers; the roles of gender and action plans

Chapter 9 General discussion 

CHAPTER 1

\section{General Introduction}

Abstract

This chapter introduces the computer generated, tailored smoking cessation expert system further studied in this dissertation. Furthermore, it provides an elaboration concerning the structure and theoretical background of the expert system, as well as an introduction to further chapters. 
Smoking kills. Although the use of tobacco can be traced back to 600-1000 AD (a Guatemalan pottery vessel depicting a Maya smoking a roll of tobacco leaves tied with a string dates from this period), only in 1761 did scientists start to make the connection between smoking and disease when dr. John Hill performed the first clinical study concerning tobacco and its effects on the human body (Hill 1761). He concluded that users of snuff (nasal consumption of powdered tobacco) were more vulnerable to nasal cancers. However, it would take until 1956 for the first major studies regarding the health effects of tobacco smoking to be conducted (Doll and Hill 1956; Wynder et al. 1956) and until 1964 for the first major report from the Surgeon General extending Hill's conclusions to the smoking of tobacco (United States Public Health Service 1964). Tobacco can be consumed through several products (e.g. chewing tobacco, snuff) and none of them are safe, but smoking is the most harmful way of tobacco consumption (Henningfield and Fagerstrom 2001). In addition, smoking has the largest impact on the health of tobacco users, as most tobacco users consume their tobacco through smoking (Mumford et al. 2006).

Previously, scientific research has been undertaken to map causal relations between tobacco and pathology; showing that smoking can affect all bodily functions (Centers for Disease Control and Prevention 2004). Furthermore, predictors of smoking uptake, cessation and relapse back to smoking have been identified (Conrad et al. 1992; Ockene et al. 2000; Ward et al. 1997). Smoking cessation was found to be predicted by demographics and smoking history, biological, situational/interpersonal and intrapersonal components (Ward et al. 1997).

Based on these predictors, methods to decrease the number of current tobacco users and prevent uptake have been developed and tested. Interventions initially consisted of communication of information regarding the negative effects of smoking on health, often within a mass media approach. An increase in knowledge and awareness was expected to lead to changes in behaviour, i.e. smoking cessation (Flay 1987). And even though this expectation was found to be correct at first, long term results were limited (USDHHS 1989). 
A shift towards influencing environmental factors to facilitate individual behaviour change became visible. Policy measures, such as tobacco purchasing regulations and smoking bans, were implemented to aid the reduction of passive smoking and smoking uptake and to encourage cessation (Prins and Willemsen 2004).

In addition, research has shown that smokers quitting without cessation aids are less successful than smokers who seek and receive help with their attempt (Zhu et al. 2000). Therefore, there is a need for effective cessation interventions on all fronts; not only measures to encourage smokers to fight the addiction (such as regulations and bans), but also to inform and teach skills to deal with situations in which it is difficult not to smoke. Computer-generated tailoring, able to provide individual smokers with personalised cessation advice, has shown to be more effective in increasing cessation rates than non-tailored materials in the past (Lancaster et al. 2000) and is therefore a promising cessation tool.

This dissertation describes an effectiveness randomised controlled trial (RCT) testing an expert system applying computer-generated tailoring technology, distributed through Dutch community pharmacies and general practices. The expert system had already been proven to be efficacious in a previous trial (Dijkstra et al. 1998d). Of importance was whether the expert system would have additional value on top of the methods and aids that are readily available within the Dutch community. Furthermore, it is of importance whether health professionals, who come in contact with smokers on a daily basis within a health care setting, are willing to use the expert system in their daily practice and what could facilitate this use. These questions call for an effectiveness trial; testing the expert system in the 'real world' (Flay and Phil 1986).

This introductory chapter will provide an overview of the considerations resulting in the development and testing of this intervention under real-life circumstances, as well as information on the content of the intervention and its theoretical background. 


\section{Planning to develop and test the expert system}

In general, smoking cessation trials present an effectiveness rate below $5 \%$ that is attributable to the intervention itself (Mudde and de Vries 1999). Because this is rather low, it is of utmost importance to ensure most optimal conditions to limit the odds of the occurrence of type II error (i.e. not detecting actual significant differences) when testing any smoking cessation intervention. A number of precautions can be taken to limit these odds by 1) recruiting a large number of respondents, 2) applying a randomized controlled design to the limit influence on outcome from other sources and 3) providing a structural plan (Kok 1992). A planning model, such as the I-Plan Model (fig 1.1)(De Vries 1998), can be used to structurally plan the development of an intervention to be as effective as possible, as the quality of the planning process determines the quality of the intervention derived from it (Kok et al. 1997).

This planning model integrates principles concerning planning and motivation and provides practical tools to enhance the model's application. Within the I-Plan Model, incorporating concepts from a number of planning models such as the PRECEDE-PROCEED model (Green and Kreuter 1991) and the Diffusion of Innovations theory (Rogers 1995), three basic phases can be distinguished: analyses of the problem, behavioural intervention and continuation. The problem analyses phase includes needs assessments for the populations of interest, identifying problem factors relating to the health problem involved, identification of target groups, analyses concerning motivational determinants of behaviour causing the health problem and access point analyses to identify how to reach the target and intermediate groups. In the second phase, behavioural intervention, objectives of the intervention are formulated. Thereafter, the intervention is developed and tested.

Providing evidence for the effect of an intervention in an experimental setting is

only the starting point however; large-scale implementation is crucial for the intervention actually taking its potential effect within a large population. This is studied in the third phase, continuation. The three phases form a continuous cycle; problem analyses can lead to the construction of behavioural interventions, which after proven effectiveness need to be embedded or implemented to continue their effectiveness. 
Findings from the continuation phase can then guide renewed problem analyses: has the problem been properly neutralized, are any additional actions required and have other problems or possible target groups emerged from the intervention's implementation. Furthermore, project activities can occur simultaneously in different phases.

\section{Problem Analysis}

Needs assessment Problem factors

Target group

Determinants

Access

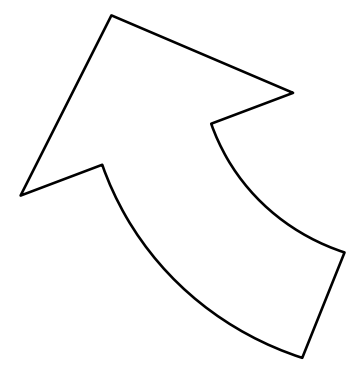

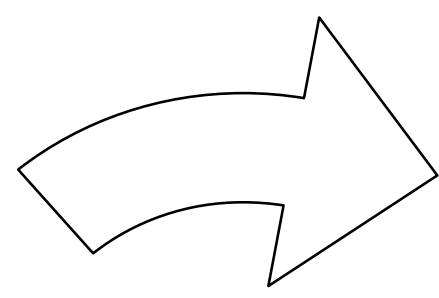

Behavioural Intervention

Goals

Methods

Testing

Continuation

Collaboration

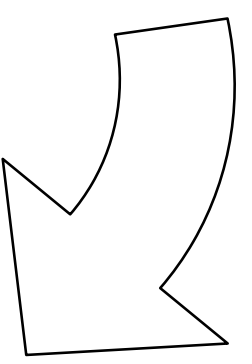

Diffusion

Policies

Fig. 1.1. The I-Plan Model (De Vries 1998).

In the rest of this introduction, the I-Plan Model will be applied to the effectiveness trial described earlier, which incorporates computer-generated tailored technology to provide smokers with a personalized cessation advice. An effectiveness study is mainly focused on the continuation phase, but is also connected to the other two phases. 


\section{Problem analysis}

Needs assessment $\mathcal{E}$ problem factors

Smoking is a major health risk; one in two smokers will die from a smoking-related condition. Additionally, smoking shortens a person's life with on average 10 years (Doll et al. 2004). In 2003, smoking was responsible for more than 20.000 deaths in the Netherlands. In addition, half of all deaths from lung cancer, COPD, coronary heart diseases and strokes after the age of 20 can be attributed to tobacco smoking (Anon 2005).

Smoking does not only affect the smoker's health; it is estimated that several thousand deaths from lung cancer and cardiovascular diseases are caused by passive smoking in the Netherlands on a yearly basis (Anon 2003a). These estimates do not include harm through morbidity. And although the number of smokers has decreased over the years since the negative effects of smoking tobacco on health have been more widely known; today still more than one in four is a smoker $(30 \%$ of the population of 15 years and older). Of these smokers, more than $25 \%$ can be classified as heavy smokers, smoking more than 20 cigarettes on a daily basis (Centraal Bureau voor de Statistiek 2006). Although the male smoking rate has been decreasing rapidly $(44.0 \%$ in 1989 , $39.4 \%$ in $1997,33.8 \%$ in 2005$)$, the percentage of women smoking was stagnating somewhat, but has now started to decrease as well $(31.3 \%$ in $1989,32.3 \%$ in $1997,25.3 \%$ in 2005) (Centraal Bureau voor de Statistiek 2004; 2006).

Summarizing, smoking is a major health threat. This threat can be taken from the health equation by decreasing the number of smokers in the community. However, quitting smoking is a difficult task, with an estimated $7 \%$ chance of reaching long-term abstinence (Baillie et al. 1995) for each quit attempt. Effective aids and encouragements are needed to help smokers kick the habit. Several means can be applied to reach this target: prevention of smoking onset, policy regulations (e.g. increasing prices of tobacco products, limiting tolerance of smoking in public places), increasing knowledge and social norm by national smoking cessation campaigns and helping current smokers kick their habit by providing effective interventions. These interventions can focus on a number of cessation methods, such as hypnosis, acupuncture or self-help books 
(STIVORO for a smokefree future 2006). However, these methods have not yet been proven to result in a significant effect on smoking cessation. Nevertheless, a number of methods aimed at individual smokers have been shown to be effective in the past and recent scientific studies, including pharmacotherapy and behavioural interventions (Hughes et al. 2004b; Lancaster and Stead 2005a; Lancaster and Stead 2005b).

The most widely used kind of pharmacotherapy is Nicotine Replacement Therapy (NRT), resulting in 1.77 times more abstinence from smoking than no intervention in efficacy trials (Silagy et al. 2004). Smokers can choose from a variety of products, such as gum, pads and spray. NRT provides the smoker with an alternative source of nicotine, which is the main addictive component in tobacco. These products are readily available over the counter in the Netherlands. However, serious nonadherence limits the effectiveness of these products (Wiggers et al. 2006). Alternatively, bupropion, an anti-depressant, can be utilized as a pharmaceutical method to quit smoking. Good results in favour of bupropion have been found in several clinical trials (Hughes et al. 2004b). However, pharmacotherapy is aimed at individual smokers willing to quit, whereas behavioural interventions can be directed towards all smokers (including those not intending to quit in the near future) (De Vries and Brug 1999). Furthermore, none of the pharmacotherapy available in the Netherlands is covered by a health plan. Therefore, the costs (on average $€ 240$ for 12 weeks of NRT, $€ 162$ for 9 weeks of bupropion) will have to be paid by the individual smoker. This could cause reluctance towards trying and maintaining to use these products. This is confirmed by previous studies showing an increase of smoking cessation treatment uptake when costs are reimbursed (Kaper et al. 2005). However, this might also be due to a novelty effect (Green and Lewis 1986), as such an intervention is a relatively new phenomenon in the Netherlands.

Behavioural interventions, such as counselling (Lancaster and Stead 2005b) and personalised written information (Skinner et al. 1999), have shown to be effective in reducing the number of smokers. In addition, pharmacotherapy requires both a financial commitment from the smoker and the involvement of a health professional, behavioural interventions do not. Its design is therefore more flexible. Furthermore, a behavioural 
intervention can easily be combined with other effective cessation methods, such as pharmacotherapy or additional behavioural interventions, as well as be provided as a stand-alone intervention.

Within behavioural intervention development, an interesting shift has occurred from providing general advice to the masses to delivering personalized messages to individual smokers. These messages can be provided during counselling sessions, but most applications have included computer programmes. These programmes offer a digitalized decision tool as to which advice is most suitable for an individual's situation, described earlier by this individual.

Computer-generated tailoring, as these interventions are referred to, have shown to be effective interventions when attempting to change a number of health behaviours, including smoking uptake prevention (Ausems et al. 2002), healthy nutrition (Oenema et al. 2005) and increasing physical activity (Smeets et al.), as well as limiting patient delay with regard to early detecting of cancer (De Nooijer et al. 2002). In addition to its positive effects on behaviour change, tailored information is more likely to be read, remembered and considered as personally relevant by its receivers (De Vries and Brug 1999; Kreuter et al. 2000).

Specifically regarding smoking cessation, extensive evidence is available that computer tailored interventions can have a larger effect than interventions providing general information or no information (Borland et al. 2004; Dijkstra et al. 1998a; Dijkstra et al. 1998d; Etter and Perneger 2001; Lancaster et al. 2000; Strecher 1999; Velicer and Prochaska 1999). Regarding the expert system described and tested in this thesis, a first efficacy study resulted in $22 \%$ quitters among smokers motivated to quit within a month (preparers) at 10 weeks follow-up, compared to 8\% in the control group (Dijkstra et al. 1998d). However, this $14 \%$ difference was not significant. A second efficacy study targeting all different types of smokers showed a positive effect of a single computer tailored feedback letter (51.4\% quit attempts, $4.8 \%$ continued abstinence) in comparison to no information (42.8\% quit attempts, 1.6\% continued abstinence) (Dijkstra et al. 1998b) at follow-up 14 months afterwards. No significant differences were found between experimental and control group with regard to point prevalence abstinence. In light of 
these outcomes, the Dutch Foundation on Smoking and Health (STIVORO for a smokefree future) implemented the intervention on a national level. However, the effect of the intervention had not yet been tested in an effectiveness trial, providing information on the additional effect on diminishing the number of Dutch adult smokers the intervention might have. Therefore, a need to assess its value under real life circumstances became apparent.

\section{Target group identification}

Every smoker is at risk to develop health problems caused by smoking. With regard to the effectiveness trial, this would imply providing an intervention for the Dutch community as a whole. However, youngsters and adolescents would require a different approach, also incorporating prevention of smoking uptake and using different recruitment channels, and would be likely more responsive to interventions specially focusing on these age groups. Furthermore, the intervention in question had already been tested among Dutch adult smokers in an efficacy study. Other than the age limitation (18 years or over), the target group was further defined by their smoking behaviour: having smoked at least once in the last 7 days. The target group therefore was defined as regular smoking adults. No additional inclusion specifications were formulated, as the computer tailoring component facilitated the intervention's ability to adjust its information to specific individuals' situations (Dijkstra and de Vries 1999).

\section{Motivational determinants}

Several theoretical models can be used to map the psychological determinants of behaviour change. One of these models is the I-Change Model (fig 1.2) (De Vries et al. 2003), the theoretical framework for the research described in this dissertation. Its predecessor, the ASE Model (De Vries et al. 1988), has been applied within several previous Dutch studies concerning smoking and other health behaviours (Ausems et al. 2002; Bolman and de Vries 1998; De Nooijer et al. 2003). 


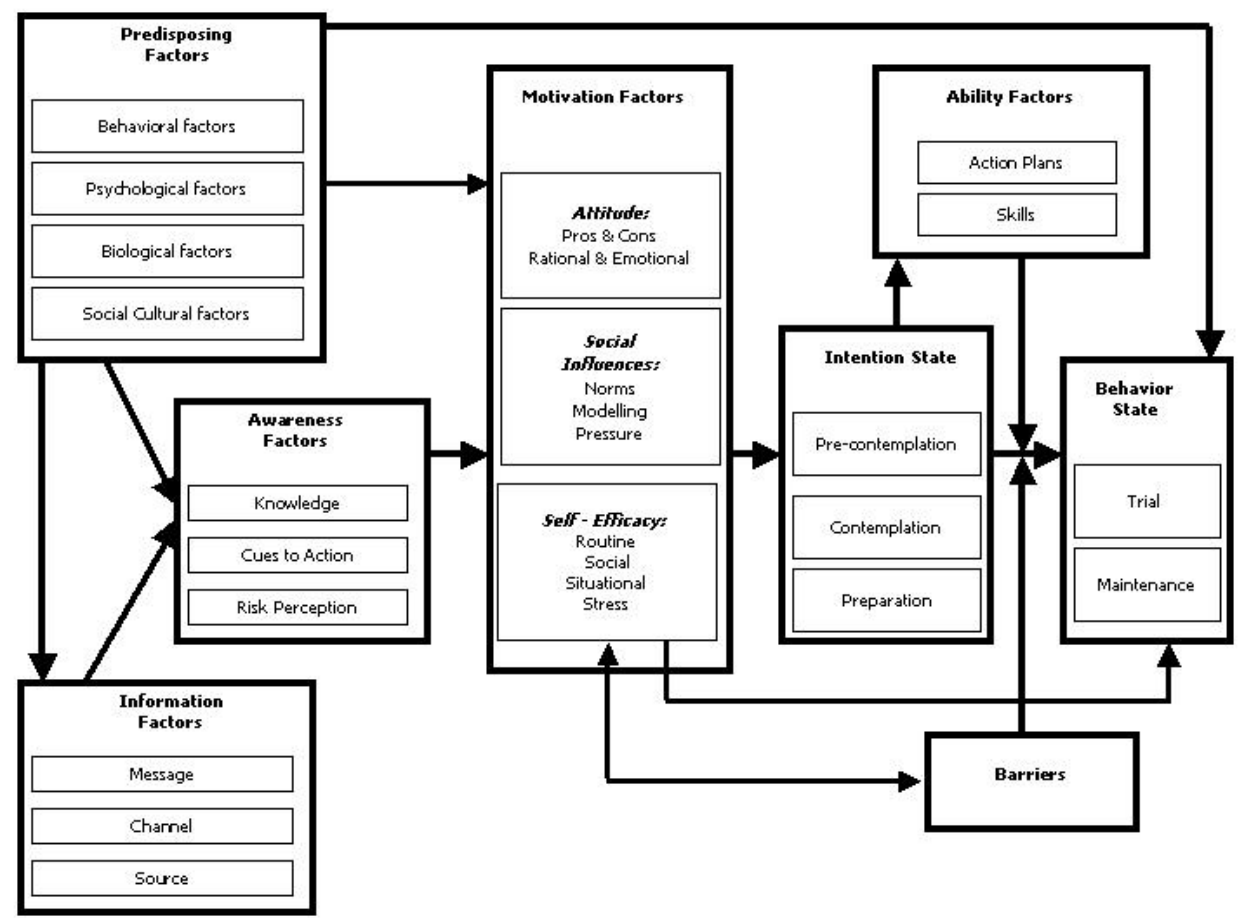

Fig 1.2. I-Change Model, version 1.31 (De Vries et al. 2003).

The I-Change Model incorporates concepts from several cognitive models, such as the Transtheoretical Model (Prochaska et al. 1997) and the Theory of Reasoned Action (Fishbein and Ajzen 1975). The concepts included in the I-Change Model have been constructed and tested within European populations; the model is therefore most suitable as a theoretical background for this intervention. The model can be applied to each of the three phases in the I-Plan Model, as long as an individual's behaviour is under study.

Centrally positioned in the I-Change Model are three main motivational concepts predicting intention (stage of change) to behavioural change: attitude, social influence and self-efficacy. These concepts are included in many models and theories attempted to predict behaviour, although appellation can differ. Attitude consists of perceived advantages (pros) and disadvantages (cons) of a specific behaviour. It can be measured by assessing beliefs involving the behaviour. Social influence incorporates 
three elements: social norm, social pressure and modelling. Social norm refers to the perceived opinions of important others around the respondent, such as family, friends and partner. Social pressure is regarded as a respondent's perceived pressure from important others to behave in a certain way. Modelling refers to perceived behaviour of others around the respondent. These elements can be measured by assessing beliefs involving the influence of the social environment on a specific behaviour. Self-efficacy refers to a person's level of confidence that a specific behaviour can be performed and is measured by assessing perceived capability to perform and maintain a specific behaviour. These three core elements are influenced by predisposing factors (behavioural, psychological, biological, and social-cultural factors), awareness factors (knowledge, risk perception and cues to action) and information factors (the quality, channel and source of information concerning the behaviour change). Eventually, abilities (skills and action plans) and barriers an individual encounters determine whether a specific behaviour change will actually be realized.

The I-Change Model states there is a discrepancy between intending to perform a specific behaviour and actually performing the behaviour: the intention-behaviour gap. Intention is conceptualized in accordance with the stages of change paradigm from the Transtheoretical Model (Prochaska et al. 1997). This paradigm categorizes respondents based on the time period within which they intend to change their behaviour: (pre-contemplation (do not want to quit within six months), contemplation (want to quit within six months) and preparation (want to quit within2 one month). The Transtheoretical Model states that each transition from one stage to another can be predicted by a unique set of concepts. For instance, pre-contemplators are thought to transition to stages closer to action (i.e. smoking cessation) if having a positive attitude towards smoking cessation. Preparers on the other hand will only attempt to quit when their self-efficacy to quit is increased additionally. These differences in predictor sets imply that interventions should be different for each stage; attitude enhancement strategies for pre-contemplators and self-efficacy enhancing techniques or skills for preparers. The stage of change paradigm has received much criticism from prominent research groups over the years (Bandura 1997; Etter and Sutton 2002; Farkas et al. 1996; 
Sutton 2001; Weinstein et al. 1998). Main concern with the paradigm is that the stages might in fact be pseudo-stages; arbitrary cut-off points hiding a continuous intention concept. However, limited longitudinal studies are available regarding predictors of forward (movement towards the action stage) and backward (movement away from the action stage) transitions. We therefore aimed to answer the following questions regarding the stage of change paradigm:

1. Does the stage of change paradigm provide a good classification measure to differentiate between sub-sets of smokers, based on intention to quit?

2. Do motivational concepts predict both forward and backward transitions through the stages?

Stage transitions of particular interest are those from the action stage to pre-action stages (i.e. relapse), as the majority of quitters will fall back and restart their smoking habit (Baillie et al. 1995). The following questions are therefore of interest:

1. What are the predictors of relapse after a quit attempt?

2. Do these predictors differ for men and women?

3. Do action plans provide a protective factor against relapse?

\section{Access point analysis}

The designated target group covers a wide spectrum of smoking adults; different age, education groups and genders. Therefore, access points should be sought out through which it is possible to reach such a diverse group of smokers. Many smokers from different subgroups can be reached through mass-media campaigns using the Dutch media. However, this form of intervention is more suited for increasing knowledge or serving as a cue to action, but rarely directly results in a behaviour change (Cavill and Bauman 2004; McVey and Stapleton 2000). Although many people can be reached, personal relevance will be low.

It can be argued that familiar surroundings based on health care (such as general practices and community pharmacies), where smokers would accept questions 
and comments concerning their smoking habit as reasonable, would increase both reach and effectiveness of a smoking cessation intervention. In addition, previous calculations have shown that the average Dutch GP sees around 670 adult smokers on an annual basis (Van den Berg 2003).

Furthermore, a Cochrane review has shown that a brief advice from a GP is an effective intervention to increase smoking cessation (Lancaster et al. 2000). These findings suggest that general practices could be an effective channel to reach smokers. However, GPs have reported that they have limited time, skills and resources to provide every consulting smoker with smoking cessation advice (Hoving et al. 2006a). The computer tailored intervention might therefore be very suitable to implement in general practice, as it gives GPs the opportunity to provide each of their smoking patients with a personalized advice without major investments in time and skills training.

Concerning the community pharmacy as an access point to reach smokers, limited information concerning its usefulness is available in the Netherlands. Nevertheless, pharmacies in the Netherlands sell pharmacotherapy aids to quit smoking and staffs are therefore used to discuss smoking cessation within these parameters. Most pharmacy staffs have a positive attitude towards patient education, although it is hardly implemented as a daily practice (Pronk et al. 2002; Pronk 2001). Accordingly, pharmacies may provide a possible additional channel to offer smoking cessation interventions.

However, the possibilities for smoking cessation interventions in the community pharmacy setting need to be assessed. Therefore, both general practices and community pharmacies were chosen as the access points or providers to reach our target group. Of interest was whether these access points would agree to use this intervention, the smoking cessation tailored expert system. Based on this uncertainty, the following questions were formulated:

1. Are Dutch general practitioners motivated and intending to adopt the expert system in their general practice?

2. What are the determinants of this intention to (not) adopt? 
Furthermore, the reach of these access points regarding recruitment was unclear, as well as the impact of the access point on recruited samples and the samples' representativeness in comparison with the Dutch smoking population. Two recruitment strategies, active and passive recruitment, could be considered. Active recruitment implies an active role of staff members; passive recruitment leaves the initiative of engaging in an intervention to the individual smoker. As limited data is available on the effect of recruiting through general practitioners or general practices on the acquired sample (Geraets et al. 2006; Harris et al. 2003; Karwalajtys et al. 2005) and no studies were detected regarding recruitment in community pharmacy, research questions regarding this topic were:

1. Do the Dutch general practices and community pharmacies settings provide good channels to recruit adult smokers?

2. Does an active role of practice staff in recruitment have an impact on the number and characteristics of recruited smokers?

3. Are samples recruited through Dutch general practice and community pharmacy representative of the overall Dutch adult smokers?

\section{Behavioural intervention}

\section{Objectives}

Another objective of this thesis was to test the effectiveness of a computer tailoring smoking cessation expert system previously found to be efficacious, providing individual smokers with personalised advice concerning their habit. The expert system was tested in Dutch general practice and community pharmacy setting.

\section{Development of the expert system}

Computer tailored interventions, such as the expert system, usually consist of three elements: a screening instrument to collect the user's information concerning the specific behaviour, a message file consisting of selections of information for all possible answers collected in the screening instrument and a computer programme combining both by 
means of decision rules (Dijkstra and de Vries 1999). The main advantage of such an intervention is the flexibility to adjust information to an individual's specific situation, which is sketched by the screening instrument. It therefore only provides information that is relevant for the individual, discarding any additional information not applicable to that specific person. Subsequently, tailored information is more likely to be read, remembered and considered as personally relevant by its receivers (De Vries and Brug 1999; Kreuter et al. 2000). Previous studies testing the effect of computer tailored interventions under ideal circumstances (efficacy studies) have shown significant results compared to no information or a generic intervention (Borland et al. 2004; Ryan and Lauver 2002; Shiffman et al. 2000). However, these results should be confirmed in effectiveness studies, in which the concepts of reach, adoption, implementation and maintenance can be studied additionally (Glasgow et al. 2003).

This dissertation deals with such an effectiveness study; the effect of the computer tailored expert system is tested under real-life circumstances. The expert system had already been developed and tested in an efficacy study, under optimal conditions (Dijkstra et al. 1998b). No substantial changes were made before testing it in this effectiveness trial. The following paragraph will give a quick overview of the expert system.

The expert system's screening instrument was a paper-based questionnaire, consisting of 54 questions, collecting information on demographic and motivational determinants, as well as intention to change the behaviour (i.e. quit smoking). The expert system was originally part of the first wave of computer tailoring interventions: using a written screening instrument and providing paper-based feedback (Sugg Skinner et al. 1999). Respondents would receive the feedback at their home address within seven working days. The screening instrument is currently only available online and can be accessed from the Dutch Foundation on Smoking and Health website (STIVORO for a smokefree future 2006).

The message file was the provider of the information: a large database of messages containing information suitable for specific answers in the screening instrument. For instance: if a smoker indicated contemplating difficulties to refrain from 
smoking in a specific situation, the output would contain a message reporting that the situation was indicated as possibly difficult by the smoker and would concern dealing with this situation. If the smoker did not contemplate difficulties in this situation, the information would not be selected in the output.

The expert system's computer programme consisted of decision rules that combined specific answers from the screening instrument with specific messages dealing with this answer, as well as software that combined these messages into readable output: a 5-7 page letter. Message content was adapted to the individual's intention to change smoking behaviour. In addition, information concerning specific attitudes and selfefficacy situations was tailored on item level, according to answers provided in the screening instrument. Decision rules were also constructed to connect messages with specific answer combinations in the screening instrument, for instance a combined message to address more than one situation in which an individual expected difficulties to refrain from smoking.

\section{Testing of the expert system}

Smoking cessation interventions, when effective, can have an impact on population health by increasing the number of years in good health and reducing premature death rates. It is therefore of the utmost importance that such interventions should be designed and tested using sound and robust research designs (Glasgow et al. 2003). First, experiments can be conducted to test the influences of concepts used in the cessation intervention. Second, an efficacy study is appropriate, in which the effects of the complete intervention are tested under optimal conditions. This usually includes a selfselected group of smoking respondents, not necessarily representative for the entire smoking population. Third, an effectiveness study can be conducted, testing the effects of the intervention under real life conditions. This also provides an opportunity to study adoption and implementation issues, which determine the reach of the intervention (Flay and Phil 1986).

A robust design to use in both efficacy and effectiveness studies is the randomized controlled trial (RCT). This design allows for the effect of the intervention to 
be tested against a control group, a group of participants not receiving the intervention: the influence of naturally occurring changes in smoking behaviour is neutralized. Participants are assigned at random to one of the groups to provide two comparable samples. A randomized controlled design is considered to be the golden standard (Concato et al. 2000). When properly executed, RCTs are less prone to external and internal validity threats (Green and Lewis 1986) than quasi-experimental and other designs. Therefore, the effectiveness trial was conducted within a randomized controlled design.

Effectiveness of the intervention was expressed as significant differences between experimental and control group in three outcome measures: quit attempt, point prevalence abstinence and continued abstinence. Respondents would be classified as having attempted to quit when indicating to have not smoked for 24 hours between baseline and a follow-up measurement. Point prevalence would indicate refraining from smoking in the last seven days before follow-up, while the continued abstinence measure would express no smoking between baseline and follow-up at all.

The main goal of this specific study was establishing point prevalence and continued abstinence, increasing the number of quit attempts was a minor objective. The main research questions regarding the expert system were therefore:

1. Does the expert system have an effect on point prevalence and continued

abstinence in a sample of Dutch adult smokers in a real-life setting?

2. Does the expert system have an effect on cessation rates in a sample of Dutch adult smokers in a real-life setting?

An intervention effect on point prevalence abstinence of $14 \%$ was demonstrated at 3 month follow-up in the efficacy study among a group of adult smokers (Dijkstra et al. 1998d). As we did not expect to find the same results in an effectiveness trial, we estimated conservatively that this difference would be found in the number of quit attempts. A power analyses indicated that for this effect to be significant, each study arm 
would have to consist of 93 smokers in preparation (willing to quit within a month) at 3 month follow-up (one-sided test, power 0.80, $\alpha=.05$ ). Estimating drop-out at 25\%, total number of smokers to recruit in preparation was 232 respondents. Assuming that $8 \%$ of the smokers at that time were in preparation, 2900 smokers would have to be recruited. As preparers represent the smallest of the overall smoker population $(8 \%)$, these calculations would also allow for a sufficient number of contemplating and precontemplating smokers to be recruited. Smokers were recruited through Dutch community pharmacies (study A) and general practices (study B). As not all smokers approached would want to participate in the study, we estimated that $35 \%$ of the smokers would participate, leaving the total number of smokers to be approached at 8286.

\section{Continuation}

In order for an intervention to be effective, it needs to be applied. The impact of an intervention can be defined as reach $x$ effect $x$ implementation (Glasgow et al. 2003); emphasizing the importance of implementation by the desired target and intermediate groups. Successful adoption and implementation can be reached in part by organising intersectional collaboration and developing diffusion and supportive strategies (De Vries 1998). However, before implementation commences, the intermediary groups appointed to provide the intervention to the target group will have to be willing to start using or adopt the intervention. A leading theory in the field of intervention implementation is the Diffusion of Innovation Theory (Rogers 1995). Rogers states that within the decisionmaking process of diffusion, five stages can be distinguished: knowledge (exposure to the intervention's existence), persuasion (formation of an attitude towards the intervention), decision (or adoption), implementation (putting an intervention to use) and confirmation (reinforcement or reversal of the implementation decision). Furthermore, five intervention characteristics related to a successful diffusion are described: relative advantage, compatibility, complexity, triability and observability (Rogers 2002). However, although an excellent framework to guide intervention design, this theory is mainly focused on characteristics of the intervention itself. Previous 
research has shown that individual characteristics and cognitive processes among potential adopters and implementers are also important determinants of adoption and implementation (Bolman et al. 2002; Paulussen et al. 1995; Reubsaet et al. 2004; Segaar et al. 2006). We were therefore interested in the following topics:

1. What are the experiences of pharmacy assistants, general practitioners and

general practice assistants with the use of the expert system?

2. Will they be willing to continue using the expert system?

3. How will they continue using the expert system?

\section{Intersectional collaboration}

In addition to research staff, initiation and continuation of an intervention depends on the cooperation and motivation of other stakeholders, which should be identified and included in a project group (De Vries 1998). Within the project group a number of stakeholder groups can be established: a research group (responsible for research, intervention and material provision), an intermediary group (responsible for providing the intervention to the target group), the target group, a support group (providing support in implementing the intervention) and a finance group (monitoring the intervention's costs). Stakeholders group's attitudes and opinions can be of direct influence on whether or not contact with the target group (adult smokers) would be successful in these health care settings. Stakeholders within this effectiveness study included Maastricht University, STIVORO for a smokefree future, the Royal Dutch Association for the Advancement of Pharmacy and the Dutch College of General Practitioners.

\section{Diffusion strategies}

Previous studies have shown that GPs indicate that they do not have enough time to engage in prevention activities on a large scale (Yarnall et al. 2003). Therefore, the expert system was designed to impose as little as possible on pharmacy and general practice staff, leaving only the first initiative to the staff after which the intervention itself was 
distributed to the smokers at their home address. Furthermore, little extra skills were necessary to use the expert system and each participating pharmacy and general practice received ample study materials from the research team. As the expert system has dealt with hindering factors staff experienced with interventions in the past, the staffs' experiences and opinions of the expert system should be recorded to be able to identify the barriers staff was confronted with. When aware of the intervention's stimulating and hindering factors, strategies can be applied to diminish or eradicate disadvantages and improve or emphasize the advantages, thus improving the intervention's implementation and increasing the odds of further diffusion among selected providers.

\section{Supportive strategies}

The effect of an intervention can not be judged without looking at interventions related to the same problem behaviour, in this case smoking. Intervention is used here as a broad term, also including policy and other actions related to smoking and smoking cessation. Since 1988, tobacco availability is limited and selling tobacco products to minors under the age of 16 , as well as tobacco advertising is prohibited by Dutch law. Furthermore, smoking is prohibited in all public places except designated smoking areas (Prins and Willemsen 2004). Also, research has not only shown that smoking damages the smoker's own health, but also that of people in the vicinity (Anon 2003a). This creates a society with a relatively negative attitude towards smoking, which could encourage smokers to seek cessation aids, increasing demand and subsequent reach of smoking cessation interventions, including the expert system. The Dutch Foundation on Smoking and Health is committed to continue providing the expert system through their website (STIVORO for a smokefree future 2006). However, no specific actions were taken to improve uptake of the expert system in community pharmacies and general practices. Furthermore, the extra attention smoking and smokers are getting through decrees and interventions could limit smokers' readiness to discuss their smoking and decrease reach of this specific intervention. 


\section{Outline of the dissertation}

The chapters in this dissertation report on the effectiveness of a smoking cessation tailored expert system in Dutch general practice and community pharmacy. Chapters 2-8 will address all three phases of the I-Plan Model; problem analysis, behavioural intervention and continuation. Additionally, results from these three phases guide analysis conducted in a second problem analysis phase.

This dissertation will discuss the following research questions:

1. What determines dissemination (adoption and implementation) of the expert system in Dutch general practice?

2. How do community pharmacy assistants evaluate using the expert system and are they motivated to further implement the expert system?

In chapter 2, the determinants of GP's adoption of the expert system are discussed. Chapter 4 and 5 describe experiences of staff from both settings with the expert system by means of telephone interviews (pharmacy assistants) and written evaluation questionnaires (GPs and practice assistants).

3. What is the effectiveness of the expert system within community pharmacy and general practice setting?

Chapter 3 describes the effect evaluation of the expert system in both general practice and community pharmacy setting among a group of smokers motivated to quit within six months (in the contemplation or preparation stage).

4. How do recruitment method and setting affect the samples acquired and how do these samples relate to the Dutch adult smoking population?

Chapter 6 describes the impact of recruitment method and setting on acquired samples. The sample acquired by active recruitment in community pharmacy setting will be compared to samples passively recruited in pharmacy and general practice setting. Differences in predisposing and motivational determinants will be discussed. 
5. Can smokers in different stages of change be differentiated from each other by cognitive factors and do different sets of cognitive factors predict different stage transitions?

In the $7^{\text {th }}$ chapter, predictors of both forward and backward transitions through the stages of change are presented.

\section{What are smokers' cognitive predictors of relapse after a quit attempt?}

Chapter 8 discusses the predictors of relapse after a quit attempt.

In the final and $9^{\text {th }}$ chapter, results from all previous chapters will be combined and discussed. Figure 1.3 provides an overview of the chapter presented in this thesis in the context of the I-Plan Model's three phases.

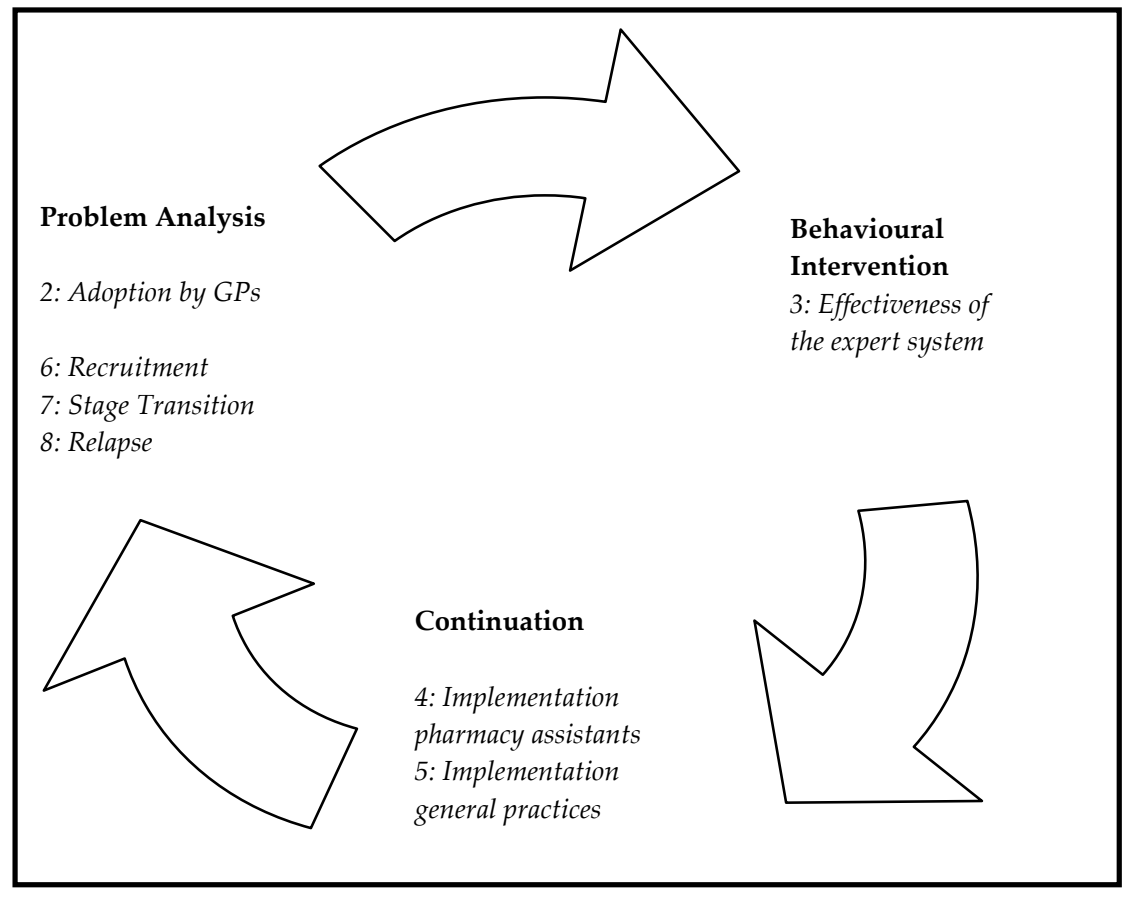

Fig. 1.3. The I-Plan Model incorporating the subjects of chapters 2-8. 
CHAPTER 2

\section{Intention to adopt a smoking cessation expert system within a self-selected sample of Dutch general practitioners}

Abstract

To investigate intention to adopt a new smoking cessation expert system as well as outline perceived barriers by general practitioners (GPs) to adopt this expert system, a written questionnaire was sent to 771 registered GPs. Respondents, representing 34.8\% of the registered GPs, were classified as adopters (34.2\%), doubters (36.2\%) or nonadopters (29.2\%). Adopters and doubters were less negative about the time investment for the GP when adopting the expert system than non-adopters. Adopters expected a more positive reaction from their patients than non-adopters. Smoking cessation support was mostly considered to be a task for the practice assistant. The authors discuss the relevance of barriers mentioned not to implement the expert system and give recommendations for further steps into implementing primary prevention activities in Dutch general practice.

Published as: Hoving $C$, Mudde AN, de Vries H. Intention to adopt a smoking cessation expert system with a self-selected sample of Dutch general practitioners (2006). European Journal of Cancer Prevention, 15(1), 82-86. 


\section{Introduction}

Advice from health professionals, such as general practitioners (GPs), is an effective way to help individual smokers to quit (Lancaster et al. 2000; Slama et al. 1999); health related advice from a GP is considered very valuable by the patient (Wensing et al. 2002). Also, GPs have the potential to reach large numbers of smokers: the average Dutch GP sees around 670 adult smoking patients at least once per year (Centraal Bureau voor de Statistiek 2003; Van den Berg 2003).

However, GPs report that barriers such as lack of time and fear of endangering the doctor-patient relationship obstruct them in assisting smokers to quit on a larger scale (Yarnall et al. 2003). An expert system that avoids these barriers by limiting GP's time investment and leaving the initiation of using the expert system to the patient might be more workable for GPs in their daily practice.

The use of expert systems is not uncommon in the Dutch general practice. Most GPs use expert systems assisting them in diagnosing and treating their patients, which are part of the computer software packages for use in general practice (Anon 2001). A special form of an expert system is computer tailoring. The concept of computer tailoring has been successfully used in studies focusing on the effectiveness of interventions targeting health-related problems such as smoking, exercise and diet (Brug et al. 1999; Bull et al. 1999; Strecher 1999). Research showed that a Dutch computer-tailoring programme led to significantly more quit attempts and had a significant positive effect on continuous abstinence (Dijkstra et al. 1998b), in line with other studies on computer tailoring for smoking cessation (Strecher 1999). This computer-tailoring programme uses written questionnaires to collect baseline information, consisting of 54 questions on smoking behaviour and opinions on smoking and smoking cessation. Respondents send their questionnaires to a central processing centre, the answers given are used to generate a personalised letter, which the respondents receive at their home address (De Vries and Brug 1999).

Even though computer tailoring proved to have a positive effect on smoking cessation rates, Dutch GPs' readiness to adopt this type of expert system has not been the 
subject of studies as yet. This is a distinct gap in research, as interventions successful in controlled circumstances do not necessarily show the same results in real life circumstances. Glasgow et al. describe the impact of an intervention as Effect $x$ Reach $x$ Implementation (Glasgow et al. 2003). The adoption and implementation of programmes by intermediates, such as GPs, have therefore an important influence on the impact of interventions. If the adoption process is not successful, then the intervention will not reach its full potential. However, the decision whether or not to adopt an innovation is subject to many factors. Rogers' Diffusion of Innovation Model (Rogers 1995) describes 5 attributes of a new idea (innovation) that are important for the adoption of this innovation: perceived benefit of adopting the innovation, compatibility with the current status (beliefs, needs, values of possible adopters), simplicity of the innovation, trialability (whether a possible adopter is allowed a trial without any obligation) and observability of the innovation elsewhere.

In order to assess the factors influencing adoption we followed the strategy proposed by Paulussen et al. (1995), who used the main concepts of attitude, social influence and self-efficacy to measure cognitive determinants influencing the intention to adopt AIDS curricula by Dutch secondary school teachers. This strategy has also been used by Reubsaet et al. when evaluating the adoption of an organ donation programme on Dutch secondary schools (Reubsaet et al. 2004). The concepts that Paulussen proposes are present in many cognitive models, such as the TransTheoretical Model and the Theory of Reasoned Action. These main concepts are also apparent in the I-Change Model (De Vries et al. 2003), the theoretical framework for this study. The I-Change Model originated from several cognition models and describes the process of behavioural change and the determinants that relate to this change. The model focuses on perceptions of the individuals eligible for adoption of the innovation, rather than Rogers' theory that focuses mainly on the characteristics of the innovation itself (Paulussen et al. 1995). The present study aimed to investigate which cognitive determinants are related to GPs' intention to adopt a computer-generated tailored 
smoking cessation expert system by comparing 3 groups of GPs, ordered by intention to adopt the expert system in their practice.

\section{Methods}

Procedure

A total of 771 GPs, registered with two District General Practitioner's Associations (DHVs) in the southern part of The Netherlands, were invited by mail in February 2003 to adopt the expert system for a period of at least eight weeks. The District General Practitioner's Associations in question provided addresses and gender details. Registered GPs from 512 general practices received a leaflet, a one-page questionnaire with accompanying letter and pre-paid envelope for returning the questionnaire. The leaflet provided general information on the expert system. Adopting the expert system meant that GPs had to agree to place a separate stand containing 20 CT-programme questionnaires with pre-paid envelopes and pens, along with 2 different posters in their waiting room for a period of at least 8 weeks. A contact person in each adopting practice was to monitor the number of questionnaires taken from the stand and to replenish when necessary. No other additional tasks were appointed to either GP or practice assistant. GPs who sent back the questionnaire were contacted by telephone to retrieve their age and experience in years as a GP. Year of graduation was retrieved for all 771 GPs (Anon 2003b).

\section{Questionnaire}

The questionnaire sent to the general practitioners was designed to measure determinants influencing the decision to adopt the expert system and was based on questionnaires used in similar studies working with the I-Change Model or its predecessor (Bakker et al. 2003; Bolman et al. 2002). It consisted of 3 questions on practice characteristics: location (rural or urban environment), practice type (solo, duo or group practice) and amount of registered patients (less than 1000, 1000-1500, 1500-2000, 2000-2500, 2500-3000, more than 3000). An additional 13 questions on the main cognitive 
variables were added: 9 questions assessing attitudes (perceived pros and cons of adopting the expert system, opinions on smoking cessation activities in the general practice), 3 questions on social influence (perceived social norm from colleagues, reactions from colleagues and patients) and 1 question concerning self-efficacy expectations (perceived possibility to adopt the expert system in our practice). The group of 13 questions had a 5-point answering scale (range: -2=disagree; 2=agree).

Respondents were also asked to indicate if they intended to adopt the expert system (I want to adopt, I need more information before I decide whether or not to adopt, I do not want to adopt), as well as to describe the main reason for this decision in their own words. Additionally, respondents were asked to indicate if GP(s) and practitioners' assistant(s) make a joint decision whether to participate in research projects or if either group alone makes this decision. The two participating District General Practitioner's Associations supplied information on gender of the registered GPs.

The letter accompanying the questionnaire encouraged respondents to return the questionnaire, even if they did not want to adopt the expert system. A total of 175 questionnaires were returned, representing practices employing a total of 262 registered GPs (34.0\%). Although some questionnaires were returned on behalf of the practice as a whole (more than one GP), all questionnaires were considered as individual responses in the analyses. A total of 55 questionnaires could not be used in further analyses (44 questionnaires contained more than $38.5 \%$ missing values on the 13 main cognitive variables, 9 questionnaires were filled in by a practice assistant and 2 questionnaires were filled in by GPs who had already adopted the expert system on their own initiative), leaving 120 questionnaires fit for analyses.

\section{Analysis}

Data from this study was analysed with SPSS 11.0. As the study aimed to give specific and practical recommendations concerning the adoption process of the expert system, variables were analysed on item level. Respondents were assigned to one of three groups 
based on their intention to adopt the expert system: adopters (I want to adopt), doubters (I need more information before I decide whether or not to adopt) and non-adopters (I do not want to adopt). Oneway ANOVA's with Tukey's HSD ad hoc comparisons were used to analyse possible differences between these groups for continuous or interval variables. Differences in dichotomous and nominal variables were tested by means of $\chi^{2}$.

\section{Results}

Non-response

No significant differences in gender or year of graduation were found between GPs who had sent back the questionnaire $(N=175)$ and those who had not $(N=569)$. When comparing the data from the questionnaires used in further analysis $(n=120)$ with those that contained too many missing values on the main cognitive variables $(n=44)$, no significant differences in gender, year of graduation or practice characteristics (working in a solo or shared practice, rural/urban environment and the number of registered patients) were found.

Sample

Our sample $(n=120)$ consisted of 99 male GPs and 21 female GPs. Mean age was 48 years, the average working experience was 18 years. GPs working in practices located in a rural area $(n=57)$ comprised $47.5 \%$ of the sample while the rest of the GPs worked in a practice in an urban environment $(n=63)$. The majority of practices $(n=96,80 \%)$ reported to have more than 2500 registered patients. Our sample showed that $48 \%(n=58)$ of the GPs worked in a solo practice (versus $61 \%$ in the population wide), $36 \%(n=43)$ within a duo practice (versus $26 \%$ population wide) and $16 \%(n=19)$ in a group practice (versus $13 \%$ population wide).

In $65.0 \%(n=78)$ of the cases, the GP decided whether or not to adopt the expert system. GP(s) and practice assistant(s) made a joint participation decision in $21.7 \%(n=26)$ of the cases, while the remaining $13.3 \%(n=16)$ did not answer the question. Most mentioned reasons for adoption were supplementing current cessation activities $(n=16$, 
$13.3 \%)$ and the health benefits of smoking cessation $(n=8,6.7 \%)$. Most mentioned reasons not to adopt were practical objections (lack of time, other priorities) $(n=24,20.0 \%)$ and doubts about the effect of computer tailoring $(n=4,10.0 \%)$. Forty-three respondents $(35.8 \%)$ did not answer this question.

Differences between adopters, doubters and non-adopters

Non-adopters $(n=35,29.2 \%)$ represented practices with significantly less registered patients than adopters $(n=41,34.2 \%)$ and doubters $(n=44,36.7 \%)(\mathrm{F}=6.20, \mathrm{p}<.01)$. No significant differences between groups were found regarding personal characteristics (gender, age and years of experience as GP), location (rural or urban environment) or practice type (solo, duo or group practice). Differences regarding attitudes, social influence and self-efficacy are presented in table 2.1.

With regard to attitudes towards adoption, adopters were significantly more positive of the usability of the expert system in their general practice than non-adopters. Both adopters and doubters believed that adopting the expert system would take up less work from the GP than non-adopters did. However, only adopters indicated adoption would require less work from the practice assistant, compared to non-adopters. Adopters felt that adopting the expert system, overall, would cost less time than doubters and non-adopters did. No significant differences were found for perceived effectiveness of the expert system, incidence of and tasks in smoking cessation activities for both GPs and practice assistants.

With regard to social influence, adopters expected a less negative reaction from their patients then non-adopters. No significant differences were found in the way respondents perceived the opinion of both other GPs and practice assistants concerning the role of the GP in smoking cessation activities.

Examining self-efficacy, both adopters and doubters were more confident that it would be possible to adopt the expert system in their general practice than nonadopters. 
Table 2.1.

Significant differences in mean scores between adopters, doubters and non-adopters on attitude, social influence and self-efficacy concerning the adoption of the expert system (scored from $-2=$ disagree to $2=$ agree).

\begin{tabular}{|c|c|c|c|c|c|}
\hline & Adopter & Doubter & $\begin{array}{c}\text { Non- } \\
\text { adopter }\end{array}$ & Differences $^{1}$ & $\mathrm{~F}$ \\
\hline \multicolumn{6}{|l|}{ Attitude } \\
\hline $\begin{array}{l}\text { The expert system is a good } \\
\text { method to use in the general } \\
\text { practice }\end{array}$ & 0.90 & 0.52 & 0.40 & $\mathrm{~A}>\mathrm{N}$ & $2.28^{*}$ \\
\hline $\begin{array}{l}\text { Adopting the expert system } \\
\text { means little extra work for the } \\
\text { general practitioner }\end{array}$ & 0.63 & 0.45 & 0.00 & $\mathrm{~A}, \mathrm{D}>\mathrm{N}$ & $5.78^{* *}$ \\
\hline $\begin{array}{l}\text { Adopting the expert system } \\
\text { means little extra work for the } \\
\text { practitioner's assistant }\end{array}$ & 0.51 & 0.30 & -0.06 & $\mathrm{~A}>\mathrm{N}$ & $5.07^{* *}$ \\
\hline $\begin{array}{l}\text { Adopting the expert system } \\
\text { for at least } 8 \text { weeks will cost } \\
\text { much time }\end{array}$ & -0.39 & 0.14 & 0.20 & $\mathrm{~A}<\mathrm{D}, \mathrm{N}$ & $6.96^{* *}$ \\
\hline \multicolumn{6}{|l|}{ Social Influence } \\
\hline $\begin{array}{l}\text { Our smoking patients will } \\
\text { appreciate the general practice } \\
\text { adopting the expert system }\end{array}$ & 0.73 & 0.55 & 0.20 & $\mathrm{~A}>\mathrm{N}$ & $5.25^{* *}$ \\
\hline \multicolumn{6}{|l|}{ Self-efficacy } \\
\hline $\begin{array}{l}\text { It is possible to offer the expert } \\
\text { system in our general practice }\end{array}$ & 1.46 & 1.07 & -0.29 & $\mathrm{~A}, \mathrm{D}>\mathrm{N}$ & $33.79^{* *}$ \\
\hline
\end{tabular}

${ }^{1} \mathrm{~A}=$ adopters, $\mathrm{D}=$ doubters, $\mathrm{N}=$ non-adopters

${ }^{*} \mathrm{p}<.05,{ }^{* *} \mathrm{p}<.01$

\section{Conclusions and discussion}

The GP's role concerning this expert system is that of an intermediate. As intermediates are the key to reach the target population, it is highly important to have insight into the determinants of adoption of this expert system. This study focused on the relation between GPs' perceptions and their intention to adopt this expert system.

Adopters had a more positive attitude towards the usefulness of the expert system, their smoking patients' reaction and time-investment by practice employees than non-adopters. A positive view on the innovation was also found as a determinant 
of adoption in previous research concerning adopting other health education tasks by GPs (Yarnall et al. 2003). According to the I-Change Model, a higher intention to change is characterised by a more positive attitude towards that change, a stimulating social influence concerning the change and a higher self-efficacy or confidence to be able to make the behaviour change. It appears that these concepts are also applicable with respect to GPs' intention to adopt a smoking cessation expert system.

Workload and time constraints seem to be important barriers for GPs to adopt the expert system. This is confirmed by what GPs indicate when asked to describe the main reason for not adopting the expert system in their own words: $20 \%$ indicated they did not have the time or had other priorities. As the response rate was limited, it is likely that the GPs in the population who were more interested in smoking cessation activities beforehand were also more likely to respond. It is therefore plausible that the percentage of GPs who feel that workload and time restraints or having other priorities are the main reasons for not adopting a smoking cessation innovation may be considerably larger. When comparing 2 Dutch national studies conducted in 1987 and 2001, it is shown that more Dutch GPs tend to work part-time but that the numbers of patients per GP have increased, which can be partly responsible for the perceived increased workload (Van den Berg 2003). Mentioning other priorities as barriers for adoption might refer to either focusing on cure rather than care (in this case engaging in prevention activities), or performing other preventive actions than smoking cessation advice. The latter is less likely, as studies previously conducted have shown that smoking cessation prevention is considered by GPs as an important activity and part of their job.

Nevertheless, as GPs feel work-related pressure is increasing; they may prioritise curative activities above prevention. Research has shown that GPs feel uncomfortable providing preventive care because they feel they lack the skills to be effective (Bruce and Burnett 1991; Tomlin et al. 1999). Discarding activities that one believes not to perform effectively seems a logical step. Although adoption of the expert system would not result in an active role for the GP, it is possible that GPs felt that 
prevention would take them away from the curative tasks they should be performing (Silverentand 2003), and therefore indicate other priorities.

\section{Limitations}

This study is also subject to limitations. Firstly, the sample used for this study is a selfselected sample. Even though GPs were also encouraged to send the questionnaire back if they did not want to adopt the expert system, the response rate was moderate. One might speculate that non-adopting GPs responding to our invitation were different from those who did not respond. However, we received equal amounts of questionnaires from adopters, doubters and non-adopters; not only GPs ready to adopt the expert system were responding. In addition, the sample was also representative for practice size.

Secondly, in order not to jeopardise response rates, the questionnaire was relatively brief (McAvoy and Kaner 1996), and may therefore not have included all relevant beliefs concerning the adoption of the expert system.

Lastly, as this study was cross-sectional, it was in principle impossible to determine causal relationships between the perceptions assessed and the intention to adopt the expert system. However, because actual adoption and experiences with the expert system took place at a later stage, these cannot have influenced opinions about the expert system. Moreover, questions concerning the decision whether or not to adopt the expert system were situated at the end of the questionnaire. This might have prevented an influence of these answers on the answers to the questions concerning the determinants of the intention to adopt.

\section{Recommendations}

Results indicate that GPs feel they are too busy to adopt the expert system and have other priorities. Nevertheless, $77.5 \%$ of the respondents indicated that they view engaging in smoking cessation activities in general as a task for the practice assistant. Delegating the task of the implementation of the expert system to others within the 
practice should decrease the barrier of time constraints, at least for the general practitioner. Yet, the consent from the persons carrying out these activities is crucial, as otherwise they might become unmotivated to engage in smoking cessation activities and the impact and frequency of these activities will likely decrease. Future research should therefore also involve practice assistants in order to establish their motivation and intention to adopt smoking cessation activities.

As the general practice can play an important role in primary prevention, it may not be prudent to turn away from GPs as providers of preventive interventions. Nevertheless, this study shows that adjusting a primary prevention programme to the demands and perceived barriers of its potential users does not necessarily imply that these barriers will disappear and that adoption is certain. GPs need to be made aware of their unique position in the Dutch care system and the possibilities that position offers for preventive activities, as well as the health benefits from providing even the most minimal amount of advice to their smoking patients. Including an accredited prevention course in Dutch GPs' curriculum, encouragement from peers and providing GPs with options to at least partly delegate smoking cessation activities could encourage a more positive attitude towards primary prevention, as well as increase self-efficacy towards engaging in prevention activities.

In addition, expectations about the GP's role in primary prevention should be realistic. Providing cessation advice to all smoking patients might sound like a good way to go about it, actually getting GPs to carry out this idea immediately might prove to be difficult. Many studies report that GPs feel more comfortable with providing smoking cessation advice when the patient asks for it or has a complaint related to smoking (Coleman et al. 2000). Although providing smoking cessation advice to all smokers might be preferable from a health promotion point of view, reaching the stage where every Dutch GP would systematically discuss smoking cessation when there is cause to do so would already be a step forward. GPs' intention towards primary prevention can be changed, but results should not be expected overnight. 

CHAPTER 3

\section{Effectiveness of a computer-generated tailored smoking cessation expert system in Dutch community pharmacy and general practice setting}

Abstract

Main aim of this study was to test the effectiveness of a computer-generated tailored smoking cessation expert system, distributed through 75 general practices and 65 community pharmacies in the Netherlands. Respondents willing to quit within six months were randomised to receive either a single tailored letter (experimental condition) or a thank you letter (control condition), after filling out a baseline questionnaire. Main outcome measures were a quit attempt, point prevalence abstinence and continued abstinence. The effect of use and appreciation of the tailored letter was also tested. In community pharmacy (CP; 3 and 12 months follow-up), 545 respondents and 474 respondents from general practice (GP; 6 month follow-up) were included. At 12-month follow-up (CP) quit attempts differed significantly between both conditions. No main effects were found in the GP sample. Both reading the tailored letter more than once (GP) and talking with others about the tailored letter (CP) were borderline significant predictors of quitting and point prevalence abstinence, respectively. Although this intervention showed positive effects in a previous efficacy study, hardly any significant differences were found between both conditions in this effectiveness study. Possible explanations for the results found in this effectiveness study are brought forward. Possible improvements of the expert system are also discussed.

Submitted as: Hoving C, Mudde AN, de Vries H. Limited effect of a computer generated tailored smoking cessation expert system in Dutch community pharmacy and general practice setting; results from two effectiveness trials. 


\section{Introduction}

The smoking of tobacco continues to be a major, yet preventable cause of illness and premature death. The Surgeon General concluded that smoking 'harms nearly every organ of the body, causing many diseases and reducing the health of smokers in general' (Anon 2004). However, this physical harm is partly reversible. Quitting smoking therefore improves both life expectancy and quality of life.

In order to stimulate and aid smokers to quit, many different smoking cessation interventions have been developed and tested subsequently. Some of these interventions have taken information relevance into account by adjusting their information supply to characteristics of specific smoker target groups or individuals. Adjustments include supplying information personally relevant for a limited group (e.g. informing pregnant women concerning the damage of smoking to the unborn child), personalizing otherwise general information (e.g. inserting the user's name in an otherwise general brochure) and providing feedback on demographic or motivational determinants supplied by an individual user at an earlier stage (e.g. providing feedback concerning beliefs previously expressed by the smoker). The latter method is referred to as tailoring.

Tailoring as such is not a new method to help smokers to quit, as interpersonal counselling also tailors to the individual's characteristics. As opposed to more static tools to provide information, such as leaflets and mass media campaigns, tailoring adjusts the information to the individual's situation and therefore only provides information that is relevant for the individual and may discard irrelevant information. Subsequently, tailored information is more likely to be read, remembered and considered as personally relevant by its receivers (De Vries and Brug 1999; Kreuter et al. 2000).

Health professionals, such as general practitioners (GPs) and community pharmacists, are in the position to provide interpersonal counselling. Additionally, previous research shows that advice from GP's is an effective intervention to help people stop smoking (Lancaster et al. 2000). However, there are constraints on effective health promotion for health professionals, such as lack of time, skills and financial reimbursements for preventive health education activities (Yarnall et al. 2003). Consequently, interventions in primary care that require only minimal input from health 
professionals are more likely to be implemented. Tailored interventions using computer technology fit this description.

Computer technology can be applied to generate tailored letters, which are part of the first generation of computer-generated tailored interventions that supply feedback in the shape of printed materials. Studies conducted previously show that printed tailored interventions perform better than interventions using a one-fits-all approach for a number of health behaviours (Brug et al. 1996; Lancaster et al. 2000). An efficacy study concerning the tailored intervention also used in our study (Dijkstra et al. 1998b) has lead to significantly more 24-hour quit attempts than no information in a group of smokers not willing to quit within six months (pre-contemplators). However, in efficacy studies interventions are delivered under optimum conditions. The additional value of effectiveness trials over efficacy trials lies in the fact that an intervention's effect is tested in the 'real world', including all possible confounding factors.

A small number of studies have been conducted previously to test the effectiveness of a smoking cessation computer-tailoring programme in general practice (Scott Lennox et al. 2001; Strecher et al. 1994), with mixed results, but to our knowledge no studies have tested tailoring programmes facilitating quitting smoking in community pharmacy setting as yet.

Therefore, main aim of this study was to determine the effectiveness of a computer-tailored smoking cessation expert system providing written feedback and distributed through Dutch general practice and community pharmacy setting. We hypothesised that a single tailored intervention letter of only a few pages of information would lead to more quit attempts than usual care among a sample of smokers motivated to quit and consequently to more abstinent smokers. Additionally, it was tested whether the respondent's perceptions of quality and usefulness of the tailored feedback were related to quitting behaviour and abstinence during the study period. 


\section{Methods}

Recruitment and procedure

Smokers were recruited during a three-month period in 2000 via 65 Dutch community pharmacies and during eight months in 2002 via 75 general practices. In 50 community pharmacies, pharmacy staff engaged in active recruitment (asking clients whether they smoked and if they wanted to participate in the study); in the other 15 pharmacies and all general practices, passive recruitment (baseline questionnaires offered in the waiting area) was conducted.

Smokers were offered a $50 \%$ chance on a computer-generated tailored advice on smoking and smoking cessation. Respondents were randomly assigned to either the experimental group (these smokers received the computer-generated tailored advice) or the control group (these smokers received a thank you letter only). Adults who had smoked in the last seven days were suitable for participating in the study. Smokers who were willing to give their informed consent were then asked to fill in a baseline questionnaire.

To make the information available for research purposes, an informed consent form was included in the questionnaire. Constant availability of baseline questionnaires in each practice was ensured by monthly replenishing visits from a member of the project staff and the appointment of a contact person within each practice to monitor the availability of the questionnaires in between visits. No other additional tasks were appointed to either GP or practice assistant. Randomisation was performed on respondent level.

Respondents in the experimental condition received a computer-generated tailored letter within seven working days after the processing centre received their baseline questionnaire. Respondents in the control condition received a letter thanking them for participating in the study within the same time frame. Follow-up was conducted by telephone six months after baseline. Ethical approval was obtained from the joint ethical committee of the Maastricht health board and Maastricht University. 
The expert system

After respondents filled in the baseline questionnaire and signed the informed consent, they were instructed to send the questionnaire to the processing centre using the prepaid envelope. Once received in the centre, the collected information from the questionnaires was entered into a database and served as input for the computertailoring expert system.

The expert system was developed by the University of Maastricht in collaboration with the Dutch Foundation on Smoking and Health (STIVORO for a smokefree future) and is currently offered to the public by the latter organization via their website. The I-Change Model comprised the theoretical framework for the expert system; incorporating several cognitive models, such as the Transtheoretical Model (Prochaska et al. 1997) and the Theory of Reasoned Action (Fishbein and Ajzen 1975). The expert system consisted of a feedback message library and a number of decision rules. The feedback library comprised a large number of messages; each designed to address a possible answer category or groups of answers given in the baseline questionnaire. The decision rules directed the tailoring programme as to which feedback messages should appear in the feedback letter, therefore only provided relevant information in the feedback. Messages addressed perceived advantages and disadvantages of smoking cessation and anticipated difficult situations to refrain from smoking. Additionally, the tailored letter was personalised by including individual information (respondent's name, the number of cigarettes smoked per day and the number of years smoked). All personally relevant messages were then combined into a five to seven page coherent letter, providing information in a logical order.

\section{Baseline questionnaire}

The written baseline questionnaire has been tested experimentally in previous studies (Dijkstra et al. 1996; Dijkstra et al. 1998b) and consists of 54 questions. Using this questionnaire demographic data (gender, age, addiction level, the occurrence of cardiovascular and respiratory diseases), number of previous quit attempts, intention to 
quit smoking and action plans concerning smoking cessation, as well as motivational determinants (attitude, self-efficacy and social influence) was collected.

Addiction level was measured with 3 items, using the abbreviated Fagerström scale (Heatherton et al. 1991): measuring tobacco product smoked, daily amount of tobacco smoked (in number of cigarettes) and expired time between getting out of bed and smoking (within 5 minutes, between 6 and 30 minutes, 31-60 minutes and after 60 minutes). Respondents' answers were scored on a 7-point scale on basis of their answers $(0=$ not addicted, $6=$ highly addicted $)$. This scale was used in analyses.

The occurrence of cardiovascular and respiratory diseases was measured by one dichotomous item each (I suffer from a cardiovascular disease, I suffer from a respiratory disease; $0=$ no, $1=y e s)$. Two items, asking respondents if they had ever seriously tried to quit smoking and how often they had attempted to quit, assessed previous quit attempts.

Questions concerning the motivational determinants attitude and self-efficacy were formulated as statements; participants were asked to indicate on a 5-point scale to what extent they agreed with the statement.

Attitude was assessed using 19 items ( $0=$ disagree/do not know, $1=$ somewhat agree, $2=$ agree, $3=$ fully agree). Factor analysis revealed three separate factors: positive outcome expectations of quitting or pros (e.g. improved own health and that of others, financial benefit, 11 items, $\alpha=.80-83$ ), negative cognitive consequences or cognitive cons of smoking cessation (e.g. weight gain, boredom, withdrawal symptoms, 5 items, $\alpha=.53-$ 59) and emotional or affective cons of smoking (dissatisfaction, shame and regret, 3 items, $\alpha=.72-75)$.

Self-efficacy was measured by 16 items (-2=disagree, $2=$ agree): respondents were asked to indicate if they would be able to refrain from smoking in emotional ( 4 items; $\alpha=$ $.82-85)$, social (4 items, $\alpha=.85-87$ ) and habitual (4 items, $\alpha=.87$ ) situations and when confronted with negative outcomes of the cessation attempt ( 3 items, $\alpha=.60-64$ ).

Two items assessed social influence measuring smoking status of the partner (yes, no, not applicable) and residential children (all, the majority, half, a minority, none, not applicable). The items were each transformed into 3 category-scales for inclusion in analyses: (-1 non-smoking, 0 not applicable, 1 smoking). 
Intention to quit smoking was assessed with one item by asking respondents to state within what period they intended to quit smoking on a 7-point scale (within 1 month, within 6 months, within 1 year, within 5 years, at some point but not within 5 years, never quit but smoke less or use tobacco products with lower nicotine and tar levels and never quit or smoke less or use tobacco products with lower nicotine and tar levels). This scale was reduced to three categories of the stages of change: pre-contemplator (not to quit within 6 months), contemplator (to quit within 6 months) and preparer (to quit within 1 month).

\section{Follow-up measurement}

Three and 12 months after baseline, all respondents recruited through a community pharmacy (CP sample) were contacted by telephone and were asked about their current smoking status and smoking activity since the last measuring point. Respondents recruited through general practice (GP sample) were contacted once; six months after baseline and were asked the same questions. For each respondent, three attempts were made to establish contact.

Three outcome measures were assessed: making a quit attempt (yes/no), smoking status at follow-up/point prevalence abstinence (having refrained from smoking in the last 7 days, yes/no) and continued abstinence (having refrained from smoking between baseline and follow-up, yes/no). Educational level and year of birth were also assessed.

Furthermore, respondents were asked to indicate whether they had used any cessation tools $(0=$ no, $1=y e s)$ or had talked to health professionals about their smoking ( $0=$ no, $1=$ yes $)$ during the trial period.

Additionally, respondents in the experimental condition were asked whether they received a personalized letter and subsequently their use (e.g. how much of the letter did you read, 7 items, $\alpha=.42-53$ ) and appreciation (e.g. how trustworthy did you find the information in the letter, 13 items, $\alpha=.74-81$ ) of the letter on a 4-point scale, ranging from not at all (0) to very much (+ or -3$)$. 
Inclusion criteria

Respondents were included in the analyses when they adhered to the following inclusion criteria: having smoked in the last 7 days before baseline assessment, being 18 years or older and being motivated to quit smoking within six months (preparer or contemplator).

Analyses

Based on their self-reported quitting activity after baseline measurement, smokers were classified as either performing or not performing the desired behaviour (quitter vs. nonquitter and abstainer vs. non-abstainer). All tests were conducted for the CP and GP samples separately.

Firstly, z-scores were calculated for all categorical and continuous items and factors from the baseline questionnaire in order to allow for analyses concerning interaction effects. Secondly, univariate logistic regression analyses were performed to test whether randomisation of the respondents had been successful. Thirdly, intentionto-treat (treating all respondents lost to follow-up as a smoker) logistic regression analyses were performed to determine the direct effect of condition on quitting activity, point prevalence abstinence and continued abstinence during the study period. Analyses were controlled for variables significantly correlating with both condition (experimental and control) and outcome variables. Fourthly, interaction effects with condition and variables significantly correlating with the outcome variable in question were calculated by means of logistic regression analysis to identify subgroups within the sample, justifying separate analyses. In order to limit the number of tests, interactions were only computed between condition and variables significantly correlating with outcome variables and tested subsequently. Fifthly, among respondents from the experimental group who remembered receiving the tailored letter, we tested whether use and appreciation of the letter predicted quitting smoking or abstinence by means of a backward logistic regression analyses. Due to a low reliability score, all items measuring use of the tailored letter were entered individually. Lastly, as respondents were nested in community pharmacy and general practice, all logistic regression analyses but those 
conducted within the experimental group only were retested using a multilevel approach (MLwiN).

\section{Results}

\section{Samples}

In total, 545 smokers, recruited through community pharmacies (CP) and 483 smokers recruited in general practice (GP) returned their baseline questionnaire. Subsequently, $256(47 \%)$ respondents from the CP sample and 227 (47\%) respondents of the GP sample were randomised into the experimental condition. From the GP sample, six respondents were 17 years or younger, four respondents had not provided their stage of change, two respondents had died during the study period and one respondent indicated not to smoke at baseline; they were excluded from all analyses, leaving 474 respondents in the GP sample. No respondents were deleted in the CP sample. As participation in the study was on the smoker's initiative, smokers not interested in participation remained anonymous and reasons for this initial refusal could not be assessed. Table 3.1 shows baseline characteristics of both the experimental and control group, for CP and GP sample respondents separately.

Randomisation in the CP sample was successful with no significant differences on gender, age, education, addiction level, intention to quit smoking (stage of change), number of previous cessation attempts or the occurrence of cardiovascular and pulmonary diseases between respondents in the experimental group compared to those in the control condition.

In the GP sample, significantly more preparers were randomised into the experimental group (OR=1.72, $\mathrm{p}<.01,95 \%$ CI 1.20-2.49). All analyses concerning the GP sample were thereafter controlled for stage of change. Table 3.2 shows an overview of outcome measures for the total sample and for contemplators and preparers separately. 
Table 3.1

Baseline characteristics for $C P$ and GP sample respondents allocated in experimental $(E)$ and control $(C)$ group separately.

\begin{tabular}{|c|c|c|c|c|c|}
\hline & & \multicolumn{2}{|c|}{ CP sample } & \multicolumn{2}{|c|}{ GP sample } \\
\hline & & $\begin{array}{l}\mathrm{E} \\
(n=256)\end{array}$ & $\begin{array}{l}\text { C } \\
(n=289)\end{array}$ & $\begin{array}{l}\mathrm{E} \\
(n=220)\end{array}$ & $\begin{array}{l}\text { C } \\
(n=254)\end{array}$ \\
\hline \multicolumn{6}{|l|}{ Demographic variables } \\
\hline \multirow[t]{2}{*}{ Active recruitment method } & & $78 \%$ & $81 \%$ & - & - \\
\hline & Missing & 0 & 0 & & \\
\hline \multirow[t]{2}{*}{ Female gender } & & $53 \%$ & $54 \%$ & $59 \%$ & $59 \%$ \\
\hline & Missing & 4 & 8 & 4 & 2 \\
\hline \multirow[t]{2}{*}{ Age (mean) } & & 46 & 47 & 43 & 42 \\
\hline & Missing & 0 & 3 & 0 & 0 \\
\hline \multirow{2}{*}{\multicolumn{2}{|c|}{ Primary school/basic vocational school }} & $26 \%$ & $26 \%$ & $30 \%$ & $27 \%$ \\
\hline & & $48 \%$ & $45 \%$ & $48 \%$ & $48 \%$ \\
\hline \multicolumn{6}{|c|}{ Higher vocational school/college degree/ } \\
\hline university degree & Missing & 62 & 67 & 44 & 49 \\
\hline \multirow[t]{2}{*}{ Cardiovascular diseases } & & $13 \%$ & $12 \%$ & $8 \%$ & $8 \%$ \\
\hline & Missing & 0 & 1 & 2 & 2 \\
\hline \multirow[t]{2}{*}{ Chronic pulmonary diseases } & & $23 \%$ & $24 \%$ & $22 \%$ & $20 \%$ \\
\hline & Missing & 0 & 0 & 3 & 3 \\
\hline \multirow{2}{*}{ Smoking partner } & & $32 \%$ & $40 \%$ & $40 \%$ & $39 \%$ \\
\hline & Missing & 2 & 2 & 0 & 4 \\
\hline \multirow[t]{2}{*}{ Smoking residential children } & & $11 \%$ & $14 \%$ & $12 \%$ & $11 \%$ \\
\hline & Missing & 1 & 2 & 1 & 5 \\
\hline \multicolumn{6}{|l|}{ Smoking behaviour } \\
\hline \multicolumn{2}{|l|}{ Cigarettes } & $48 \%$ & $51 \%$ & $54 \%$ & $50 \%$ \\
\hline \multicolumn{2}{|l|}{ Cigarette tobacco } & $27 \%$ & $30 \%$ & $27 \%$ & $28 \%$ \\
\hline \multicolumn{2}{|l|}{ Cigars } & $2 \%$ & $2 \%$ & $1 \%$ & $2 \%$ \\
\hline \multicolumn{2}{|l|}{ Pipe tobacco } & - & $1 \%$ & - & - \\
\hline \multirow[t]{2}{*}{ Using $>1$ type of tobacco } & & $24 \%$ & $17 \%$ & $18 \%$ & $20 \%$ \\
\hline & Missing & 0 & 0 & 0 & 0 \\
\hline \multicolumn{6}{|l|}{ Stage of change } \\
\hline \multicolumn{2}{|l|}{ Contemplator } & $41 \%$ & $41 \%$ & $38 \%$ & $52 \%$ \\
\hline \multirow[t]{2}{*}{ Preparer } & & $59 \%$ & $50 \%$ & $62 \%$ & $48 \%$ \\
\hline & Missing & 0 & 0 & 0 & 0 \\
\hline \multirow[t]{2}{*}{ Cigarettes smoked per day (mean) } & & 22 & 21 & 22 & 22 \\
\hline & Missing & 0 & 0 & 0 & 1 \\
\hline
\end{tabular}


Table 3.2.

Quit, point prevalence and continued abstinence percentages at follow-up per condition for the total sample and for preparers and contemplators separately.

\begin{tabular}{|c|c|c|c|c|c|c|}
\hline \multirow[b]{2}{*}{ Total sample } & \multicolumn{2}{|c|}{$\mathrm{CP}$ sample 3 months } & \multicolumn{2}{|c|}{ CP sample 12 months } & \multicolumn{2}{|c|}{ GP sample 6 months } \\
\hline & $\mathrm{E}(n=256)$ & $C(n=289)$ & $\mathrm{E}(n=256)$ & $C(n=289)$ & $\mathrm{E}(n=220)$ & $C(n=254)$ \\
\hline Quit attempt & $37 \%$ & $31 \%$ & $69 \%$ & $60 \%$ & $50 \%$ & $41 \%$ \\
\hline Point prevalence & $8 \%$ & $5 \%$ & $13 \%$ & $10 \%$ & $15 \%$ & $15 \%$ \\
\hline $\begin{array}{l}\text { Continued } \\
\text { abstinence }\end{array}$ & $4 \%$ & $3 \%$ & $2 \%$ & $2 \%$ & $10 \%$ & $9 \%$ \\
\hline Preparers & $\mathrm{E}(n=152)$ & $C(n=171)$ & $\mathrm{E}(n=152)$ & $C(n=171)$ & $\mathrm{E}(n=136)$ & $C(n=123)$ \\
\hline Quit attempt & $49 \%$ & $39 \%$ & $76 \%$ & $65 \%$ & $53 \%$ & $46 \%$ \\
\hline Point prevalence & $12 \%$ & $7 \%$ & $15 \%$ & $12 \%$ & $16 \%$ & $15 \%$ \\
\hline $\begin{array}{l}\text { Continued } \\
\text { abstinence }\end{array}$ & $7 \%$ & $5 \%$ & $4 \%$ & $4 \%$ & $13 \%$ & $11 \%$ \\
\hline Contemplators & $\mathrm{E}(n=104)$ & $C(n=118)$ & $\mathrm{E}(n=104)$ & $C(n=118)$ & $\mathrm{E}(n=84)$ & $C(n=131)$ \\
\hline Quit attempt & $19 \%$ & $20 \%$ & $59 \%$ & $53 \%$ & $44 \%$ & $37 \%$ \\
\hline Point prevalence & $2 \%$ & $3 \%$ & $9 \%$ & $8 \%$ & $13 \%$ & $15 \%$ \\
\hline $\begin{array}{l}\text { Continued } \\
\text { abstinence }\end{array}$ & $0 \%$ & $1 \%$ & $0 \%$ & $0 \%$ & $6 \%$ & $8 \%$ \\
\hline
\end{tabular}

Results from the community pharmacy $(C P)$ sample

No significant differences were found at 3 months follow-up for all three outcome measures, nor were any significant interaction effects found. At 12 months follow-up, respondents in the experimental group were more likely to have attempted to quit smoking than their counterparts in the control condition (OR 1.48, 95\% CI 1.03-2.11, $\mathrm{p}<.05)$. As number of previous cessation attempts significantly correlated with both condition and quitting smoking, the analysis was controlled for this covariate. Interaction effects were not significant and multilevel analyses showed no level effects.

Within the experimental condition, a small minority of respondents in the experimental condition could not remember receiving the tailored letter $(n=12,6 \%)$ and an additional 63 respondents were not contacted for follow-up (25\%), leaving 178 respondents fit for analysis. Talking with others about the letter reached borderline significance for point prevalence (OR 3.47, p=.07, 95\% CI 0.92-13.10) at 12 months follow- 
up. Respondents who had been discussing the tailored letter with others were more likely to be abstinent for at least seven days at the last follow-up.

Results from the general practice (GP) sample

All analyses were corrected for stage of change. No main effects were found for point prevalence and continued abstinence. For point prevalence abstinence the interaction effect for condition with smoking behaviour partner was borderline significant (OR 1.70, $\mathrm{p}=.06,95 \%$ CI $0.98-12.94)$. However, when splitting the sample on partner's smoking behaviour, no significant effect was found indicating that respondents with a smoking partner were significantly more likely to reach point prevalence abstinence at six months follow-up. Multilevel analyses showed no level effects.

Within the experimental condition, 19 respondents did not remember receiving the tailored letter and 42 respondents were not reached at follow-up, leaving 151 respondents to test the appreciation and use of the tailored letter. Reading the tailored letter more than once was borderline significantly related to making a quit attempt (OR 2.94, $\mathrm{p}=.08,95 \%$ CI $0.85-10.21)$.

\section{Cessation aids and consulted health professionals}

To test if (additional) cessation aids or consulted health professionals concerning attempting to quit during the trial period had influenced the lack of differences between both conditions, $\chi^{2}$ tests were performed for preparers and contemplators separately. Respondents in the experimental group did not significantly differ from those in the control condition on whether cessation aids were used or health professionals consulted. This was the case for all follow-ups.

\section{Discussion \& Conclusion}

This paper describes a randomized controlled trial concerning the effectiveness of a smoking cessation computer-tailored expert system in a sample recruited through Dutch general practice $(n=545)$ and community pharmacy $(n=474)$. Effect was expressed as quitting activity, point prevalence and continued abstinence. 
Although an efficacy study concerning the same expert system showed a significant positive effect on smoking cessation outcomes (Dijkstra et al. 1998b), this was not replicated in this effectiveness study. As this study was subject to real life confounders, these could explain the difference from the expert system's effect in the efficacy trial. However, that study was conducted with a sample of pre-contemplating smokers, whereas smokers included in the effectiveness study were motivated to quit. It would be in the line of expectation that these motivated smokers would be more likely to attempt to quit than unmotivated smokers.

Albeit that it can not be ruled out that the intervention is not effective outside an experimental setting, some threats to the validity of the results found are applicable here (Green and Lewis 1986) and are discussed below; lack of power to find significant results, social desirability \& testing effect, extraneous events, the availability of the intervention outside the study and data entry issues.

\section{Lack of power}

Other effectiveness studies including larger samples found significant effects of computer tailored interventions. Two studies in the United States (Strecher et al. 1994) showed a significant effect of computer-tailoring in general practice. Also, an RCT concerning the effectiveness of a fully automated internet-based smoking cessation programme among smokers ready to quit within a month (preparers) showed a $12 \%$ point prevalence rate (intention-to-treat) at follow-up after three months (Swartz et al. 2006), as in our sample. Their $x^{2}$ test, thus not including any confounding variables, showed a $5 \%$ significant difference between the experimental and control condition. The CP sample included 152-171 preparers in each condition arm; a power calculation shows that for the $5 \%$ difference to be significant, each arm would had to include 275 respondents (power $=.80)$.

\section{Social desirability}

Respondents from both conditions might have thought it socially desirable to indicate they had quit smoking, when in fact they had not. As our study did not include 
biochemical validation, it was not possible to verify respondents' claims concerning their smoking status. Nevertheless, smokers would not gain in any way other than healthrelated gain by having quit or not. Also, personal contact with research staff was severely limited, thus it is unlikely that this influenced the participants' responses at follow-ups. In addition, previous studies (Glasgow et al. 1993; Patrick et al. 1994; Velicer et al. 1992) suggest that the difference between self-reported abstinence rates and those verified with biochemical validation is negligible.

\section{Extraneous events $\mathcal{E}$ testing effect}

The baseline questionnaire could have provided all respondents with more insight in their smoking behaviour and possible strategies to change that behaviour, thereby explaining most of the effect. Additionally, respondents were tested more than once, the testing experience could make them 'test-wise' and could motivate them to change their behaviour for the next test round; independent of the intervention they did or did not receive. Also, differences in how data is collected (baseline written questionnaire, followups telephone interviews) can also change the results, regardless of the intervention received.

Furthermore, during the general practice study's follow-up period, an extensive smoking cessation campaign was started, comprising of radio and television attention, a website, telephone support, adds and special 'start quitting' packages, including the baseline questionnaire (STIVORO for a smokefree future 2003b). However, smokers who filled in the questionnaire from the package were not included in the study's experimental condition. Therefore, it is plausible that a number of respondents from the control condition from both studies have received the tailored letter, thus limiting the difference in outcome measures rates between the experimental and control condition. Nevertheless, the conditions did not differ on (additionally) used cessation aids or consulted health professionals.

The expert system was also widely available during both effectiveness trials through a number of channels (e.g. health professionals practices including general practices and community pharmacies and directly available from the Dutch Foundation 
on Smoking and Health). Although it was therefore possible for respondents in the control condition to acquire the tailored feedback, only in those community pharmacies and general practices that participated in the study extra attention was paid to promoting the expert system in terms of posters, separate folder stand, a press release and so on. Also, acquiring the expert system through another channel than the study required more initiative from the smoker. And although no respondents in the control condition indicated at follow-up to have used the expert system, this was in response to a general question whether they had been using other smoking cessation methods and respondents might not have viewed the expert system as a cessation tool.

Both the testing effect and the extraneous events might have caused a cessation ceiling effect, after which the intervention had little to no additional effect.

\section{Data entry issues}

As baseline questionnaire data entry was performed manually at the time of the study, errors in data entry may have caused minor alterations in the tailored letter, which has a large effect on the believability of personalization of the letter by the respondent. For instance, it is plausible that a male respondent receiving a letter addressing him as a woman will doubt that the rest of the letter is correctly personalized. At this moment, the Dutch Foundation on Smoking and Health has digitalized the expert system, thereby reducing the data entry error margin and improving its accessibility to a large number of smokers. In addition, intention-to-treat analyses were conducted, which included all respondents randomised at baseline and assumed drop-out respondents had remained smokers.

\section{Conclusions}

Within this study, the expert system appeared to have limited effect on abstinence in real life setting. Future studies could possibly decrease the likelihood of confounding issues as discussed above by preparing an extensive recruitment and intervention execution plan and back-up plans for both, as well as include further questions concerning use of other smoking cessation interventions or methods during the study period. 
Concerning the expert system, adaptations might be valuable to increase effectiveness and personalization of the tailoring. In the current expert system, social influence is measured by two items, focusing on modeling. However, pressure and norm of the direct social environment are also regarded to be important in behaviour change within the I-Change Model (De Vries et al. 2003).

Also, action plans have shown to have a positive effect on relapse prevention after a quit attempt (Hoving et al. 2006b). Therefore, the expert system could be more extensively measured in the baseline questionnaire; the tailored feedback could possibly provide respondents with the opportunity to phrase their own action plans. Furthermore, studies concerning multiple tailoring (providing respondents with tailored feedback on more than one occasion) have shown to have a more positive effect on smoking cessation outcome measures (Brug et al. 1998; Dijkstra et al. 1998c). Further expansion of the expert system into several moments providing iterative feedback could increase its effectiveness. 


\section{CHAPTER 4}

\section{Intention to implement a smoking cessation intervention in Dutch general practice}

Abstract

General practices can provide many smokers with cessation advice. Aim of this study was to identify determinants of general practice staff's intention to further implement a smoking cessation expert system. Written questionnaires based on the I-Change Model were left in general practices after trying out the expert system. Respondents intending to continue their use (intenders) were compared to those who did not (non-intenders). Fifty-five practices responded (73\%). GPs were more often intenders than practice assistants. Responses from the same practice were not significantly related to each other. A more positive attitude towards the expert system, social norm towards engaging in smoking cessation activities and higher self-efficacy determined intention to continue using the expert system. Practice staff who had actively offered the expert system to their patients were more likely to be an intender. Cognitive factors and involvement in the trial played a role determining intention to further implement the smoking cessation expert system. Discussing barriers with practice staff could increase motivation to implement and ownership. Intenders can play a role in the implementation process by sharing experiences with non-intending peers. Further research should study acceptable investment from practice staff and the GP's specific role in the effectiveness of smoking cessation advice from general practice. Previous research has shown that a GP's brief advice can increase smoking cessation rates. Determinants of intention to implement a smoking cessation intervention can be used to keep general practices involved in smoking cessation activities.

Published as: Hoving C, Mudde AN, de Vries H. Intention to implement a smoking cessation intervention in Dutch general practice (2007). Health Education, 107(3), 307-315. 


\section{Introduction}

Advice from general practitioners (GPs) has a significant positive effect on smoking cessation rates, even if this advice is brief (Lancaster et al. 2000). GPs recognise the importance of smoking cessation activities and indicate that these activities at least partly belong in the general practice (McAvoy et al. 1999; McEwen and West 2001). Nevertheless, GPs report barriers such as time constraints and concern about damaging the doctor-patient relationship with health education activities related to smoking cessation (Hoving et al. 2006a; Yarnall et al. 2003). They prefer to discuss cessation only with patients with smoking-related problems or patients already motivated to quit smoking (Coleman and Wilson 1999; Coleman et al. 2000; Helgason and Lund 2002; Senore et al. 1999).

These implementation barriers suggest that an innovation with a self-help nature and a minimum of effort from the GP will be of practical use in the general practice and is therefore most likely to be implemented on a larger scale. Computergenerated tailoring is an expert system that meets these recommendations. It offers general practices an opportunity to provide their smoking patients with an individualised cessation method without a specific role for practice staff. Dijkstra et al. (1998b) tested the impact of an expert system in a randomised control trial. This expert system, based on the I-Change Model, was developed by the University of Maastricht in cooperation with the Dutch Foundation on Smoking and Health (STIVORO for a smokefree future 2006). Results indicated that the expert system had a significant positive effect on smoking cessation rates in a sample of self-selected subjects.

Smoking patients who use the expert system filled in a questionnaire containing 54 personal questions on smoking and smoking cessation. This questionnaire acted as a screening instrument. Smokers sent their filled-in questionnaire by mail to the national processing centre. There, answers from the questionnaire were entered into the expert system. The expert system consisted of a feedback library with messages related to the topics in the questionnaire. Only messages relevant for the individual smoker appeared in their feedback, a written letter consisting of three to five pages. This feedback was then sent to their home address. 
In order for the expert system to reach smoking patients, it has to be implemented by GPs and their staff. As this study was partly focused on cognitive factors of intention to implement an innovation, a model in which these factors are more prominently present was used as the theoretical framework for this study; the I-Change Model (De Vries et al. 2003). This model incorporates several cognitive models, such as the Transtheoretical Model (Prochaska et al. 1997) and the Theory of Reasoned Action (Fishbein and Ajzen 1975). The I-Change Model states that the intention to change behaviour is determined by predisposing factors (behavioural, psychological, biological, social cultural factors), awareness factors (knowledge, cues to action, risk perception), information factors (message, channel, source) and motivational factors (attitude, social norm, self-efficacy). The intention to change in combination with an individual's abilities and experienced barriers influence the likelihood of accomplishing the desired behaviour change. Its predecessor, the ASE Model (De Vries et al. 1988) has been previously used in studies concerned with the implementation of health education programmes (Paulussen et al. 1995).

The I-Change Model recognises that offering an opportunity to try out the expert system (trialability) could encourage the transition from adoption (planning to start using an intervention) to implementation (embedding an intervention into daily practice). This research aimed to study factors related to this transition among Dutch general practices after a trialability opportunity.

\section{Methods}

\section{Respondents and recruitment}

The general practices eligible for inclusion in this study had been participating in an effectiveness study concerning the expert system described above. For this effectiveness study, 512 general practices were asked for permission to distribute the expert system questionnaires. The questionnaires with return envelopes were displayed in a separate folder stand in the waiting room. Pens with the project logo to fill in the questionnaires were provided and two A4-size posters were displayed to draw attention. All practices were visited monthly to replenish project material when necessary. A contact person 
within the practice monitored the questionnaires taken from the stand. Practice assistants were preferred over GPs due to logistical reasons (better availability and closer ties to the waiting room). Neither GP nor practice assistant had to carry out any additional tasks. The effectiveness study served as a trialability opportunity to become familiar with the expert system. A total of 75 general practices agreed to participate in this study.

After eight months, the effectiveness study ended and two process-evaluation questionnaires were left behind in each practice. One general practitioner and one practice assistant from each general practice were invited to fill in the questionnaire. Responses were anonymous, but general practice of origin was recorded. After two weeks, a written reminder was sent by mail to all non-responders.

\section{Questionnaire}

The three-page questionnaire consisted of 29 questions concerning demographics, motivational determinants on both smoking cessation activities in general and the expert system specifically, previous smoking cessation education activities, the degree of implementation of the expert system during the effectiveness study and future action plans concerning the use of the expert system.

Seven questions measured demographic variables of the respondent: occupation (general practitioner or practice assistant), practice type (solo: one GP with assistance, duo practice: two GPs with assistance or group practice: >two GPs with assistance), practice location (rural, semi-urban or urban), smoking status (smoker, non-smoker or ex-smoker) and the percentage of registered patients who smoke (respondents could also tick a box if this percentage was not known).

The main cognitive variables (attitude, social influence/social norm and selfefficacy) were scored on a five-point scale (minus two disagree, two agree), as were previous cessation education activities, the degree of implementation during the expert system trial and future implementation plans.

Two items assessed attitudes concerning smoking cessation education in general (I think that smoking cessation education is important, I think that smoking cessation 
education has a place in the general practice, $\alpha=.55)$, whereas three items assessed attitudes towards the expert system (I think the expert system is an effective smoking cessation method, the expert system is a good addition to the smoking cessation materials we already had in use, I think the expert system is very usable in the general practice by general practitioners and practice assistants alike, $\alpha=.82$ ).

Social norm concerning smoking cessation education in general was assessed by four items (my colleagues inside/outside the practice think that smoking cessation education is important, my colleagues inside/outside the practice think that smoking cessation education has a place in general practice, $\alpha=.84)$.

Self-efficacy concerning smoking cessation activities in general was assessed with two items (I do not find it difficult to address smoking and smoking cessation, I have influence on the smoking behaviour of my patients, $\alpha=18$ ), whereas self-efficacy concerning using the expert system was assessed with four items (I will succeed to use the expert system on smoking patients who are asking for a cessation advice, I will succeed to use the expert system on smoking patients who are not asking for a cessation advice, I will succeed in supplementing the expert system questionnaires in the practice so they can always be offered, I will succeed in offering the expert system even when there are other smoking cessation methods available, $\alpha=.81$ ).

The questionnaire assessed the degree of implementation of the expert system trial with six items (e.g. during the project we kept two posters in the waiting room at all times, all employees were informed about the project, $\alpha=.55$ ).

Intention to keep using the expert system in the future was assessed by six items (I plan to order the expert system, I plan to discuss further use of the expert system within our practice, I plan to keep offering the expert system in our waiting room, I plan to actively offer the expert system to our smoking clients, I plan to stimulate colleagues within our practice and outside to use the expert system, $\alpha=.85$ ).

Analysis

Data was analysed using SPPS 11.0. Respondents were assigned to one of two groups based on their intention to use the expert system in the future: intenders and non- 
intenders. This was determined by computing a mean score of the six items concerning intention to keep using the expert system in the future; respondents with a score above zero were considered intenders, respondents with a score of zero or below were considered non-intenders.

Kolmogorov-Smirnov tests showed that all interval variables to be included in the analyses were not normally divided, indicating the need for non-parametric testing. Therefore, attrition analyses were conducted by means of in order Mann-Whitney U tests and $\chi^{2}$ tests to determine a selection bias between those who had responded and those who had not. Furthermore, differences between non-intenders and intenders were determined to reveal relations between future action plans concerning the expert system and demographic variables, main cognitive variables and the degree of implementation during the trial. To find out whether a GP's intention to further implement the expert system was related to the intention of the assistant from the same practice, a $X^{2}$ test was conducted.

In order to minimise the number of separate tests on such a limited sample, mean scores were calculated within concepts, based on the reliability scores shown in table 1. Mean scores with a reliability score larger than 0.7 were used (as suggested by Nunnally (1978)). In case mean scores had a lower reliability score, the individual item scores were entered into the analysis. Items and concepts were then introduced into a backward logistic regression analysis in three blocks with intention to keep using the expert system in the future as dependant variable; the first block contained four demographic variables (job function within the general practice, practice type, location of the practice and smoking status of the respondent), the second block contained seven items and mean scores concerning the main cognitive variables (attitudes concerning smoking cessation education in general and towards the expert system, social norm concerning smoking cessation education in general and self-efficacy concerning smoking cessation activities in general and using the expert system) and the third block contained six items concerning the degree of implementation of the expert system during the trial. Variables were excluded on basis of the change in the model's likelihood ratio. 


\section{Results}

Sample

A total of 95 respondents $(63 \%)$ returned the questionnaire, representing $73 \%$ of participating practices. Six questionnaires contained too many missing values (> 10\%) and were excluded from further analysis. Attrition analyses showed no significant difference concerning function within the practice (general practitioner or practice assistant) or practice type (solo, duo or group practice) between respondents and those who did not respond.

In the final sample, 48 respondents functioned as assistants (53.9\%) and 41 as general practitioners (46.1\%). A total of $46.1 \%(n=41)$ worked in a solo practice, $37.1 \%$ $(n=33)$ in a duo practice and $16.9 \%(n=15)$ in a group practice. Respondents represented 27 solo practices $(50.0 \%), 19$ duo practices $(35.2 \%)$ and eight group practices $(14.8 \%)$. From 33 practices, both GP and assistant responded $(59 \%$ of the 52 participating practices). Most respondents $(n=70,78.7 \%)$ did not know the percentage of smokers among patients registered with their general practice, but from those respondents $(n=19)$ who did give an answer, the mean percentage of registered smokers was $14.5 \%$. Most respondents worked in general practices in an urban $(n=35,39.3 \%)$ or semi-urban $(n=33$, $37.1 \%$ ) environment, 21 respondents $(23.6 \%)$ worked in a general practice situated in a rural area. A majority of the respondents had never smoked $(n=56,62.9 \%)$, ten respondents were currently a smoker $(11.2 \%)$ and 23 respondents were ex-smokers (25.8\%). Many respondents indicated that they intended to further implement the expert system in their general practice $(n=62,69.7 \%) ; 80.5 \%$ of the GPs and $60.9 \%$ of the practice assistants could be classified as intenders.

\section{Differences between intenders and non-intenders}

The group of intenders included significantly more GPs than the non-intender group $\left(\chi^{2}=4.22, \mathrm{p}<.05\right)$. GPs' intentions were not significantly related with those of assistants in the same practices $\left(\mathrm{X}^{2}=0.75, \mathrm{p}=.39\right)$. Table 4.1 shows the differences between intenders and non-intenders concerning attitudes, social norm, self-efficacy and degree of implementation. 
Table 4.1.

Mann-Whitney $U$ tests: differences between non-intenders (NI) and intenders (I).

\begin{tabular}{|c|c|c|c|c|}
\hline & $\begin{array}{l}\text { Number of } \\
\text { items }\end{array}$ & $\begin{array}{l}\text { Mean rank } \\
\text { NI }\end{array}$ & $\begin{array}{l}\text { Mean } \\
\text { rank I }\end{array}$ & $\begin{array}{l}\mathbf{P} \\
\text { value }\end{array}$ \\
\hline Attitude expert system $(\alpha=.82)^{*}$ & 4 & 29.98 & 51.98 & .000 \\
\hline $\begin{array}{l}\text { Attitude smoking cessation education ( } \alpha \\
=.55)\end{array}$ & 2 & & & \\
\hline - Importance & & 39.61 & 47.35 & .013 \\
\hline - Established place in general practice & & 36.37 & 48.76 & .006 \\
\hline $\begin{array}{l}\text { Social norm concerning smoking cessation } \\
\text { education }(\alpha=.84)^{*}\end{array}$ & 4 & 34.22 & 49.69 & .007 \\
\hline $\begin{array}{l}\text { Self-efficacy concerning smoking cessation } \\
\text { activities }(\alpha=.18)\end{array}$ & 2 & & & \\
\hline - Ease to discuss smoking cessation with patient & & 41.48 & 46.53 & .310 \\
\hline - Influence on patients' smoking behaviour & & 40.65 & 46.90 & .252 \\
\hline $\begin{array}{l}\text { Self-efficacy use of the expert system } \\
(\alpha=.81)^{*}\end{array}$ & 3 & 30.11 & 51.48 & .000 \\
\hline Implementation degree $(\alpha=.55)$ & 6 & & & \\
\hline - 2 posters in the waiting room & & 41.22 & 46.65 & .324 \\
\hline - Questionnaires in the waiting room & & 42.48 & 46.10 & .375 \\
\hline - Questionnaires in separate folder stand & & 43.15 & 45.81 & .554 \\
\hline - Active recruitment of smokers & & 38.89 & 47.66 & .126 \\
\hline - Appointment of a contact person & & 51.30 & 42.26 & .104 \\
\hline - Notification of all staff members & & 43.35 & 45.72 & .657 \\
\hline
\end{tabular}

* Entered into the logistic regression analyses as concepts

Intenders considered smoking cessation activities in the general practice more important and were more likely to think that smoking cessation activities had a place in the general practice. They also held a more positive attitude towards using the expert system.

Intenders also reported a more positive social norm concerning the system and were more confident about using the expert system.Table 4.2 shows the results of the logistic regression analysis.

Logistic regression analysis showed that practice assistants were less likely to implement the expert system in their practice than GPs, when correcting for general practice characteristics. After including attitude, social pressure and self-efficacy, the influence of occupation became even more apparent. A higher self-efficacy score concerning using the expert system and experiencing a positive social norm also contributed to the decision to further implement the expert system. Also, respondents 
were more prone to further implement the study if they had actively offered the expert system to smokers during the trial. The model explained $52 \%$ of the variance.

Table 4.2.

Final model from backward logistic regression analysis: differences between non-intenders (0) and intenders (1).

\begin{tabular}{lll}
\hline & OR & $\mathbf{9 5 \% ~ C I ~}$ \\
\hline Occupation (GP=1, practice assistant=2) & $0.10^{* *}$ & $0.02-0.45$ \\
Social norm concerning smoking cessation education & $4.41^{* *}$ & $1.74-11.17$ \\
Self-efficacy use of the expert system & $5.09^{* *}$ & $2.10-12.36$ \\
Implementation degree: 2 posters in the waiting room & 1.38 & $0.96-1.98$ \\
Implementation degree: Active recruitment of smokers & $1.68^{*}$ & $1.03-2.74$ \\
\hline
\end{tabular}

Nagelkerke's R square $=.52$

\section{Discussion}

GPs had a more positive intention towards implementing the expert system than practice assistants; 8 GPs versus 19 practice assistants were not inclined to further implement the expert system. Lock et al. also found that GPs involved in an alcohol screening programme had more positive attitudes towards the programme than the primary health care receptionists involved (Lock et al. 2000). This could be due to the fact that contact persons in most participating practices were practice assistants. The delegation of this task to the practice assistant might be involuntary, thus influencing the practice assistants' attitude towards the expert system. Also, as the recruitment for participating practices in the trial was focused on GPs, practice assistants may have felt overlooked. In combination with a perceived, possible involuntary, increase in workload this might have caused a less positive attitude towards the expert system and therefore less motivation to continue using the expert system after the trial in the group of practice assistants, compared to the group of GPs.

A more positive social norm towards smoking cessation activities in general practice and stronger self-efficacy expectations to use the expert system appear to positively influence the intention to use the expert system in the future as well. These findings are in line with the outline of the I-Change Model, which states that attitude, social influence and self-efficacy have an influence on the intention to change or maintain certain behaviour, in this case, the continuation of using the expert system. The 
high percentage concerning explained variance of the model supports this hypothesis. Practices that actively pointed out the possibility of using the expert system to their smoking clients were more likely to intend to keep using the expert system. It might be that a somewhat active role of practice staff increases involvement with the expert system.

This study is subject to some limitations. First, although the sample described in this paper is representative for the population of 75 general practices that were involved in a trial concerning the expert system, all practices had already agreed to adopt the expert system for the period of the trial and practice staff might therefore have viewed the expert system more positive than the average Dutch general practice would have. However, it would be more likely that the differences between intenders and nonintenders would increase or multiply rather than the other way around. The characteristics of non-intenders we found in this study are also likely to be applicable for general practices that did not even want to adopt the expert system for as long as the trial period; all the more reason to take notice of these characteristics.

Second, it is possible that staff members were influenced by each other. In 33 general practices, opinions of GPs as well as assistant could be assessed. However, in those practices, no significant correspondence between responses of GPs and assistants were found.

Third, this study focused on the intention to further implement the expert system, rather than the action of implementation itself. Even though the theoretical model we used for this study assumes that behaviour follows from the intention to perform this behaviour, it is also recognised that barriers can prevent intention to develop into action. However, the expert system was set up to diminish at least practical barriers, such as time restraints and GP's reluctance of addressing all smoking patients as much as possible.

\section{Conclusion}

This study has identified a number of determinants important in the decision whether or not to implement a smoking cessation expert system by general practice staff. 
It seems that a somewhat active role of general practice staff positively influences the intention to continue using the expert system in the future. Therefore, bypassing the practice staff entirely does not seem prudent, especially since research has shown that the GP's health advice increases smoking cessation rates.

However, these investments in time and effort might have their limits. Further research could determine investments by general practice staff needed to create some degree of ownership, but does not exceed the extent of investment staff is willing to make. Also, the specific role of the GP in the effectiveness of general practice-based smoking cessation advice should be studied, in order to determine whether the delegation of tasks to other staff members within the general practice might influence the positive effect of an advice concerning smoking cessation through general practice (Lancaster et al. 2000).

General practice staff willing to continue using the expert system can be characterized as having a more positive attitude towards the expert system, as well as a higher confidence in being able to use the expert system in the future. When we assume that the expert system is an innovation worth using by GPs, it is desirable for the expert system to be implemented by the entire population of Dutch GPs. Rogers indicates that social influence through members who have already implemented an innovation is important for the continuation of the process by the entire population (Rogers 1995). This is in line with the finding in our study that intenders perceive a social norm more towards engaging in smoking cessation activities than non-intenders.

Overburdening staff could mean that the expert system will be rejected before it is even seriously considered for implementation. If discussing these and other barriers during the development or adjustment of smoking cessation methods with the persons who will actually be responsible for implementing these methods, motivation to work with the method can be increased and the base for ownership can be created.

Although there is a difference between intention to perform certain behaviour and the actual behaviour, if intenders continue to implement the expert system, they are in the position to motivate and stimulate other general practices to start using the expert system. If the trialability of the expert system is increased, a more positive attitude 
towards the expert system can be established, as well as an increased confidence to eventually implement the expert system into daily practice. By inviting their peers who have not yet implemented the expert system for a demonstration or by sharing their experiences with the expert system, implementers might be able to increase nonimplementers' interest and self-efficacy expectations and provide a forum to address misconceptions. 
CHAPTER 5

\section{Pharmacy assistants are not ready to incorporate a smoking cessation expert system into their daily routine}

Abstract

The objective of this study was to assess the opinions of pharmacy assistants concerning their participation in active recruitment of smoking clients for a smoking cessation expert system effectiveness RCT study during a three-month period. Thirty-three telephone interviews with pharmacy assistants from 19 pharmacies previously involved in the active recruitment were conducted. Questions concerning smoking cessation activities, experiences with the recruitment and future plans concerning smoking cessation in the pharmacy were included in the interview. Respondents indicated that smoking cessation interventions were important, but did not perceive the pharmacy to be the most suitable channel. They also had trouble approaching clients without smoking-related complaints. Furthermore, the recruitment period proved to be too long and recruiting could not structurally be included in daily practice. In order to actively involve pharmacy staff in smoking cessation interventions, these barriers should be addressed. Also, skills training could increase staff's self-efficacy to approach possible smokers and discuss their smoking behaviour. Shorter recruitment periods and feedback concerning the pharmacy's recruitment accomplishments could accommodate recruitment in the community pharmacy.

Submitted as: Hoving $C$, Mudde AN, de Vries H. Pharmacy assistants are not ready to incorporate a smoking cessation expert system into their daily practice. 


\section{Introduction}

Effectively providing health promotion activities to the general public is a constant challenge. Some theoretical models, such as the I-Change Model (De Vries et al. 2003), assume that the public does not only judge the content of health promotion messages, but also takes source and channel through which these messages reach them into account. It is therefore important to stimulate a wide reach of information or interventions by identifying suitable distribution channels to reach the target population.

Health professionals can play the role of intermediates by serving as a health intervention distribution channel, either in an active or passive manner. In the Netherlands, emphasis has usually been on general practitioners (GPs), as they serve as gatekeepers of Dutch primary health care. Within general practice, a large population of adults can be reached, including smokers. It is estimated that Dutch GPs see around 670 adult smokers at least once a year (Van den Berg 2003). However, GPs have indicated that they have limited time and resources to provide each of their smoking patients with cessation advice beyond the suggestion to quit (Hoving et al. 2006a; Yarnall et al. 2003).

Another promising setting with the potential to reach a large number of smokers and provide them with cessation advice is the community pharmacy. Adding on the potential effectiveness is the fact that Dutch community pharmacies are distribution points for Nicotine Replacement Therapy (NRT) and that pharmacy staff has knowledge concerning the proper use of these products.

A number of international studies have investigated the community pharmacy as smoking cessation intervention setting. In a review, Blenkinsopp and colleagues (2003) describe two randomised controlled trials (RCT), both testing the effectiveness of a smoking cessation intervention (based on the stage of change principle) in community pharmacy setting. A counselling and weekly follow-up intervention (PAS) conducted in community pharmacies in Northern Ireland and England resulted in 14.3\% abstinence after 12 months, compared to $2.7 \%$ in the control group (Maguire et al. 2001). Although pharmacists providing smoking cessation advice in Scottish community pharmacies did result in a higher abstinence rate after nine months amongst their smoking clients than usual care, the difference was not significant (Sinclair et al. 1998). However, clients in the 
intervention condition rated their discussion concerning smoking cessation higher than clients in the control condition.

A limited number of recent studies have focused on the opinion of the pharmacy staff concerning smoking cessation activities in the community pharmacy. In a recent study mapping smoking cessation activities among pharmacists in US community pharmacies, only $7.5 \%$ reported that they routinely record their clients' smoking status, but almost half of all respondents would advise to quit smoking if a client's smoking status was known (Williams et al. 2000). However, pharmacy staff's opinion concerning using specific smoking cessation interventions in their daily practice has to our knowledge not yet been assessed.

This paper described the outcomes of telephone process evaluations with pharmacy assistants participating in a randomised controlled trial (RCT) to test the effectiveness of a smoking cessation intervention: a computer tailored smoking cessation expert system among smoking clients in community pharmacy setting. The process evaluations aimed to map previous and current smoking cessation activities in their respective community pharmacies, as well as their opinion concerning using the tailored expert system during and after the trial.

\section{Methods}

Procedure

In 2001, 50 pharmacies throughout the Netherlands participated in an RCT to test the effectiveness of a smoking cessation expert system based on computer tailoring. The expert system provided pharmacies with a means to offer their smoking clients a written personalised advice on cessation. Smoking patients filled in the questionnaire, which was then sent to a central distribution centre. There, the questionnaire's information was entered into the expert system. The expert system consists of a feedback library with messages related to the topics in the questionnaire. Only messages relevant for the individual smoker appear in their feedback, a written letter consisting of three to five pages, sent to their home address. A previous study has showed a significant positive effect of the expert system of smoking cessation rates (Dijkstra et al. 1998b). 
For a period of three months, community pharmacy staff was required to actively recruit respondents for this trial, by asking all clients at the desk if they smoke and if they would like to participate in the trial. Pharmacies were provided with the study materials; the questionnaires with return envelopes, a separate folder stand for the questionnaires (to be displayed in the waiting area), pens with the project logo to fill in the questionnaires and two A4-size posters. All pharmacies were visited by a member of the research team before the start of the three-month period. During this visit, the research design was explained and tactics on how to identify and approach smoking clients were discussed. Each pharmacy appointed a contact person who monitored the questionnaires taken from the stand and reported to the trial staff whether they needed any new materials, such as questionnaires and pens. Each month, all pharmacies were contacted by telephone by a member of the research team, who kept a log of these calls.

At the end of the recruitment period, pharmacies were asked to participate in a process evaluation study concerning the usability of the expert system in community pharmacy setting. Two pharmacy assistants per pharmacy were asked to participate in a telephone-based interview. They were informed that an interview would take 15 minutes to complete. When a pharmacy assistant consented, an appointment was made on a time convenient to them, mostly during the less busy times of the day in the pharmacy.

Both interviews and telephone logs were used to provide information on the pharmacies' smoking cessation activities before, during and after the project.

Interview routing

The interview routing was semi-structured in nature, allowing for pharmacy assistants to raise additional subjects during the interviews. Table 5.1 shows the interview question route.

Questions were based on concepts of the I-Change Model (De Vries et al. 2003), combining several cognitive behavioural models. The model describes three main motivational concepts predicting intention to change or maintain a specific behaviour: 
attitude, social influence and self-efficacy. These three core elements are influenced by predisposing factors, such as behavioural factors (e.g. previous behaviour).

Previous behaviour concerning smoking cessation education in the community pharmacy was assessed by 11 questions (e.g. Did you provide smoking cessation education to clients at risk?).

Seven questions assessed the assistants' attitude towards smoking cessation and the expert system (e.g. Is providing smokers with smoking cessation education a task of the pharmacy?).

Social influence from colleagues and clients towards smoking cessation activities was assessed by 6 questions (e.g. Do clients want the pharmacy to pay attention to smoking?).

The degree of self-efficacy to actively recruit smokers in the pharmacy was assessed by one question (Did you feel capable to actively approach the clients in the pharmacy?).

Additional activities to promote the expert system provided by the pharmacy staff were also recorded.

Two items assessed the intention to continue using the expert system and recruiting smokers in the future.

Analyses

The interviews were transcribed and loaded into the NVivo 2.0 programme for qualitative data analyses (Richards 1999). Two researchers coded the interviews according to a pre-defined coding tree, deducted from the topics discussed in the interviews. Each main theme was divided into sub-themes. Segments of the transcripts were assigned to one of the sub-themes, based on content. Cohen's kappa was calculated between theme assignments of both coders to ascertain consensus, resulting in $\kappa=0.73$, indicating a satisfactory consensus. 


\section{Table 5.1.}

Pharmacy assistant interview question route.

Previous behaviour (11 items)

Principal question: In the past, did you provide smoking cessation education?

In depth questions: Under what conditions?

- To clients asking for Nicotine Replacement Therapy?

- To clients with a smoking-related complaint?

- To clients at risk?

- To random clients?

In depth questions: When providing smoking cessation education, what elements did it contain?

- Discuss a client's smoking habits?

- Discuss a client's motivation to quit smoking?

- Discuss the risks of smoking and the benefits of quitting?

- Discuss situations where it could be difficult not to smoke?

- $\quad$ Provide the client with educational material?

- Discuss the possibilities of Nicotine Replacement Therapy?

- Refer clients to other health professionals?

Attitude (7 items)

Principal question: What is your opinion about providing smoking cessation education in your pharmacy?

In depth questions:

- It is important to provide smoking cessation education?

- Is providing smokers with smoking cessation educational a task of the pharmacy?

- Did paying attention to smoking in the pharmacy have a positive influence on the smoking habits of clients?

- Will more people quit smoking when the pharmacy pays attention to quitting smoking?

- Do you think the smoking cessation expert system was effective?

- Do you think the active recruitment was effective?

- What do you think of the duration of the project?

Social influence (6 items)

Principal question: what do you think that others think of the role of pharmacies in smoking cessation?

In depth questions:

- Does the pharmacist in your pharmacy believe that paying attention to smoking is important?

- Do the other assistants in your pharmacy believe that paying attention to smoking is important?

- How did clients respond to your enquiries?

- Do clients want the pharmacy to pay attention to smoking?

- Who made the decision to participate in the study?

- Was this decision discussed in a staff meeting? 
Table 5.1. (continued)

Self-efficacy (1 item)

Principal question: Was it feasible to actively approach the clients in the pharmacy? In depth question:

- What were the barriers in actively approaching clients?

Additional activities during the project (1 item)

Principal questions: Did you do anything additional to attract attention to the project?

Intention to continue using the expert system ( 2 items)

Principal question: Will you continue to use the expert system?

In depth question:

- Will you offer the expert system actively or passively?

\section{Results}

\section{Sample}

A total of 33 assistants were interviewed, representing 19 participating community pharmacies (38\% response rate). In 14 pharmacies of these pharmacies, two assistants were interviewed. In the other 5, only one assistant was interviewed. All but one assistant was female.

Previous behaviour

For the majority of practice assistants interviewed, before using the expert system, usual care concerning smoking cessation consisted of providing information only on the client's request, mostly in combination with a request for NRT. A small minority did provide information to clients at risk or with smoking-related complaints, but mostly not structurally. None of the assistants provided information to random clients without smoking-related questions or complaints. This was mainly due to the belief that it was the client's responsibility to ask for information about quitting smoking.

\section{“...No, I do not believe I am in the position to do so. Most of the time you get a negative} response on such a question anyway. Like 'It's not your business'... "

When providing information, they mostly discussed the possibilities of NRT and the client's smoking habits to determine the right dosage of NRT. Most assistants did not 
discuss advantages and disadvantages of quitting with smoking clients, as they assumed that the clients already knew most of the facts about smoking and quitting smoking. The majority did not ask about the client's motivation to quit or discuss difficult situations not to smoke.

In addition to oral information, the assistants usually also provided written information, consisting mostly of product information or instructions to use NRT or bupropion. Some clients were referred to other medical workers, mainly the general practitioner (GP) for a bupropion prescription (NB bupropion is not available over the counter in the Netherlands).

"...Well, some people come here when there is a new drug for smoking cessation, but you often need a receipt for that....so they need to go to their GP...but other than that, no..."

\section{Attitude}

A majority of assistants indicated that it was very important that pharmacies provide information concerning smoking and smoking cessation. However, they felt it was difficult to accomplish when under-staffed and during busy periods in the pharmacy.

“...Yes, [giving education about smoking] is very important, however we have quite a shortage of staff and that is why we don't always have the time to give attention to providing information about smoking. That's a pity..."

Even though most of the assistants highly valued smoking education in the pharmacy, only a slight majority felt that smoking cessation interventions are not primarily the responsibility of the community pharmacy, but feel that GPs, specialists and clients themselves are first responsible for providing and receiving proper smoking cessation education.

According to most of the assistants, smoking cessation interventions in community pharmacies would have a positive impact on the clients and increase the number of quitters. However, they could not estimate its impact, as they had not 
received any feedback from those clients who had participated in the intervention. Those who did not agree felt that smoking clients already receive enough information through other channels.

The majority of the assistants who participated in the project believed that through active recruitment more clients could be reached and the project would be more effective, compared to a passive approach. However, some assistants felt that the project continued for too long, which caused some clients to be approached more than once, resulting in a decline in the assistant's motivation.

\section{Social Influence}

Most assistants perceived that their pharmacist and colleague assistants viewed providing smoking cessation education in the pharmacy as important. Most assistants believed that all or some clients appreciated the pharmacy's attention to smoking cessation. In effect, the majority of the clients responded positively to the invitation to participate in the project, although some assistants did experience some negative responses. Most assistants felt that active recruitment resulted in including smokers that would not have been approached in their usual routine.

“...I do think that this way you reach a group you wouldn't normally reach that easily, because you have to ask very actively...I do think that you can persuade people this way... By asking actively you reach other people than when it would be only the initiative of the client..."

Although the pharmacist decided to participate in the study in most cases, in a large majority of the pharmacies, this decision was then discussed during a pharmacy staff meeting.

\section{Self-efficacy}

In spite of the mainly positive beliefs about the potential effectiveness of the project, a large majority felt that active recruitment was not always practical and feasible as it 
sometimes was too busy in the pharmacy and assistants experienced some difficulties asking people whether they smoked or not.

"...Most of the time it is [feasible to actively approach every client]. However, sometimes it is very busy, so that you do not have the possibility to ask every client to participate in the project, without feeling the work pressure. Especially when there are many people waiting to be helped..."

Moreover, practice assistants felt that it was inappropriate to interfere with their clients' private lives. Some assistants reported privately assessing whether a client would be interested in the project or not before approaching the client. If they felt that the client would not be interested beforehand, they would not approach this client.

\section{Activities during the project}

In a majority of the pharmacies efforts were being made to attract attention from their client's to the project. This was mainly done by means of a press release and by decorating the display window or a showcase. From the log kept by the research team, it is clear that although most pharmacies started to actively recruit smokers with enthusiasm at the beginning of the project, this dwindled shortly after.

Intention to continue using the expert system

A large majority indicated they would like to continue using the expert system, but only by using a more passive approach. Although some respondents indicated that they would like to continue approaching smokers in an active manner, they would limit this active approach to a shorter period.

"...We want to start some sort of project once in a while, for example in January or February. In those months we would hand out the questionnaires using an active approach, maybe only in January. During the rest of the year we would only have the questionnaires in case someone asks for them..." 


\section{Discussion \& Conclusion}

This article describes a process evaluation among 33 community pharmacy assistants, previously involved in an RCT concerning the effectiveness of a smoking cessation expert system.

Pharmacy assistants are aware of the benefits from smoking cessation and believe interventions concerning the subject are important and could be effective, but the smoker should make the initial step in asking for help. They also indicated that their advice was almost always related to a client's question or to determine the appropriate NRT dosage. Thereby, they are indicating they focus more on cure than on care. In addition, they felt that other health professionals, such as the GP, are better equipped to provide smokers willing to quit with sound advice. However, Dutch GPs indicated they have limited resources for these activities as well (Hoving et al. 2006a).

Furthermore, the recruitment period of three months appears to be too long to be structurally embedded in the assistants' daily routine. Assistants indicated that busy periods in the pharmacy and shortage of staff were reasons for not recruiting all adult clients. As assistants were unaware of the outcomes of the intervention concerning their recruited smokers, this caused doubts on whether their recruitment efforts paid off.

Although many pharmacy assistants were willing to continue using the expert system in the future, most would turn to limited active recruitment or complete passive recruitment. Respondents also indicated that they had difficulty with asking clients about their smoking behaviour. Although all pharmacies were visited before recruitment started and study design and were discussed, assistants might have benefited more from a training focussing on identifying and recruiting techniques. A recent US study (Zillich et al. 2004) showed that by providing pharmacy assistants with a 2-hour training, their knowledge concerning smoking cessation did not only increase, but increases in selfefficacy and outcome expectancy providing smoking cessation education were also observed. 


\section{Conclusion}

Pharmacy assistants indicate a number of barriers to actively recruit adult smokers in their community pharmacy on a day-to-day basis.

It might be unrealistic to expect pharmacy assistants to actively engage in health promotion tasks when other services must have priority at specific moments. Health promotion activities should not interfere with the core task of a pharmacy, i.e. supplying medication and information concerning these medications.

However, the community pharmacy could offer additional advice in cooperating with other health professionals, such as the GP. However, as community pharmacies look to distinguish themselves from drugstores and other pharmacies, health promotion could be a good opportunity to accomplish this. Pharmacy staff should be made aware of the possibilities of providing health promotion advice to their clients, thus offering an extra service. Training opportunities should become available for pharmacy staff in order to increase their self-efficacy concerning providing smoking cessation advice beyond explaining the information leaflet provided with medication. The effect of shorter recruitment periods and feedback reports on both pharmacy staff and recruited samples should be explored in future research. Furthermore, recruitment through community pharmacies could be embedded in larger campaigns, providing the pharmacy staff a starting point from where to commence recruitment activities. 
CHAPTER 6

\section{Effect of recruitment method and setting on the composition of samples consisting of adult smokers}

Abstract

This study aimed to determine differences in samples of adult smokers recruited by passive or active recruitment in community pharmacy ( $\mathrm{PP}$ and $\mathrm{AP}$ ) or passive recruitment in general practice setting (PG), then comparing these samples to an unrecruited cohort of Dutch smokers. The three recruited samples were compared on demographics, smoking behaviour, motivational determinants, stage of change and intention to use specific action plans when quitting using multinomial logistic regression analyses; this method was also used to assess whether recruitment effected the acquired samples concerning demographic variables compared with the cohort. PP, AP and PG response rates were compared with Tukey post-hoc tests. Significant differences were found for both AP and PG smokers, compared with PP smokers. Most important results include a higher rate of pre-contemplators in the AP sample and a lower education level in the PG sample. All recruited samples appeared to be significantly different from the cohort sample. It can be concluded that recruitment method and primary care setting does influence the sample recruited, this should be taken into consideration when recruiting a specific sub-sample. These results are valuable for researchers contemplating recruitment at a primary care setting.

Published as: Hoving C, Mudde AN, de Vries H (2007). Effect of recruitment method and setting on the composition of samples consisting of adult smokers. Pat Educ Couns, 62(1), 79-86. 


\section{Introduction}

Study samples are ideally representative of the population they are recruited from, as results derived from the sample can then be generalised to that population. To this end, a number of recruitment methods (e.g. invitations in person, by post, e-mail or telephone) can be employed to help include a representative sample into research. In addition, these methods can be used in many settings (e.g. community-wide, patient registries or health care practices), also influencing response rate and representativeness (Bero et al. 1998).

However, not all recruitment methods and settings are necessarily similar in effect (Margitic et al. 1999; McDonald 1999) and as researchers often have limited time and resources to recruit their participants, there is little room for error. Therefore, impact of recruitment methods, settings and their respective recruitment rates are important issues to consider when enrolling participants. Choosing a recruitment method fitting its purpose is time- and cost effective, as the likeliness of recruiting the desired sample increases.

Primary care health professional practices, such as general practices and community pharmacies, provide a good setting to recruit participants for health promotion research (McIntosh et al. 2000). As gatekeepers of the Dutch care system, their patient and client populations serve as good representations of Dutch care seekers concerning demographic variables such as age, education and gender. As general practices and community pharmacies are used widely in the recruitment of participants for scientific research and health promotion interventions, it is important to have an understanding of the effectiveness of these recruitment settings.

Until now, only a limited number of studies have focused on these recruitment settings. In a Dutch study, adults with chronic shoulder complaints were recruited for a behavioural treatment programme either during a consultation with their general practitioner or by advertisements in a local paper (Geraets et al. 2006). Patients recruited by either method did not differ concerning baseline characteristics, dropout rate, number of withdrawals and post-treatment clinical outcomes. However, only 83 patients were 
recruited by either method, thus generalisation is questionable. Additionally, recruitment methods were used in different settings (general practice versus community).

A study describing recruitment of African Americans into a clinical trial testing the effect of bupropion on smoking cessation (Harris et al. 2003) found that smokers recruited actively by health care providers and study staff reported a higher education and income, better health and less indicators of depression, compared to passive recruitment where information was disseminated in the community asking smokers to call a hotline to participate. However, as the methods recruited within different socioeconomic populations, this could have accounted for the significant differences found. Again, recruitment methods were used in different settings.

In a pilot to determine effective recruitment methods for older family practice patients to participate in cardiovascular health awareness sessions, no significant differences in baseline characteristics were found between patients recruited by mail or by telephone (Karwalajtys et al. 2005). Yet, as only a single practice and a limited number of patients were involved, caution is warranted when interpreting these results.

Overall, firm conclusions cannot be drawn on the basis of these studies, as research methods, settings, populations and research subjects differed within and between studies and some studies were not optimally designed. Therefore, welldesigned research is in order in which either method or setting is kept as a constant factor. Then, the differential effects of method and setting can be separated.

Aim of this study was to add to the knowledge concerning effect of recruitment method and setting by determining whether different recruitment methods (active versus passive) and settings (community pharmacy versus general practice) used to recruit smoking adults for a computer-generated tailored advice intervention would influence the characteristics of the sample acquired. This resulted in two research questions: 1) do smokers recruited in community pharmacies for a computer-generated tailored advice intervention by means of passive recruitment differ in demographic and motivational determinants from those recruited through active recruitment in the same 
setting and 2) do smokers recruited in a passive way for tailored advice in the general practice differ in demographic and motivational determinants from those recruited with the same method in community pharmacies?

Additionally, the effect of recruitment method on these determinants from acquired samples was assessed by comparing these samples with a cohort sample of Dutch adult smokers.

\section{Methods}

\section{Procedure}

Adult smokers (18 years or older) were recruited through 65 community pharmacies and 75 general practices to participate in an effectiveness study of a computer-generated tailored advice on smoking and smoking cessation. Smokers were eligible regardless of their intention to quit smoking; smokers not wanting to quit at all were invited to participate as well. Participants were asked to fill in a baseline questionnaire; the answers provided formed the basis of the tailored advice. Each participant had a 50\% chance of receiving tailored advice and was informed about this in advance.

Both active and passive recruitment methods were applied in pharmacies (50 active recruiting pharmacies, 15 passive recruiting pharmacies), in general practices only a passive recruitment method was applied. Active recruitment included staff enquiring after every customer's smoking status and inviting current smokers to participate. Upon agreement to participate, staff would present the smoker with the baseline questionnaire. Passive recruitment entailed providing the questionnaires in a separate folder stand in the waiting area, leaving uptake to the smoker's initiative. In all settings, posters were displayed in the waiting areas to attract attention and to provide a starting point for staff actively recruiting participants. All pharmacies and general practices were contacted regularly and a contact person within each participating unit monitored the questionnaires taken from the stand and replenished when necessary.

Recruitment continued for three months in the community pharmacy setting and eight months in the general practice setting. To standardise the recruitment time, 
only respondents recruited in general practice in the first three months were included in the analyses described.

\section{Questionnaire}

Baseline characteristics were collected using a written questionnaire consisting of 54 questions. This questionnaire has been tested experimentally (Dijkstra et al. 1996; Dijkstra et al. 1998b) and is currently the basis of a computer-generated tailored smoking cessation programme distributed nationwide by the Dutch Foundation on Smoking and Health (STIVORO for a smokefree future).

Demographics (gender, age, the occurrence of cardiovascular and respiratory diseases), addiction level, number of previous quit attempts, stage of change to quit smoking and action plans concerning smoking cessation, as well as motivational determinants (attitude, self-efficacy and social influence) were assessed.

Addiction level was measured with 3 items, using the abbreviated Fagerström scale (Heatherton et al. 1991): assessing the type of tobacco product smoked, the number of cigarettes smoked per day and time after tobacco is smoked when getting out of bed (within 5 minutes, between 6 and 30 minutes, 31-60 minutes and after 60 minutes). Respondents were scored on a 7 -point scale on basis of their answers $(0=$ not addicted, 6=highly addicted). This scale was used in analyses.

The occurrence of cardiovascular and respiratory diseases was measured by one dichotomous item each (I suffer from a cardiovascular disease, I suffer from a respiratory disease; $0=$ no, $1=y e s)$.

Previous quit attempts were assessed by 2 items: asking respondents if they had ever seriously tried to quit smoking and how often they had attempted to quit.

Questions concerning the motivational determinants attitude and self-efficacy were formulated as statements; participants were asked to indicate on a 5-point scale to what extent they agreed with the statement.

Attitude was assessed using 20 items ( $0=$ disagree/do not know, (-)1= somewhat agree, (-) 2=agree, (-) 3=fully agree): positive outcome expectations of quitting or pros 
(improved own health and that of others, financial benefit), negative cognitive consequences or cognitive cons of smoking cessation (weight gain, boredom, withdrawal symptoms) and emotional or affective cons of smoking (dissatisfaction, shame and regret). Higher scores on positive outcome expectations implied seeing more pros, whereas higher scores on both cognitive and emotional cons implied seeing less cognitive or affective cons.

Self-efficacy was measured by 16 items (-2=disagree, 2=agree): respondents were asked to indicate if they would be able to refrain from smoking in emotional, social and habitual situations and when confronted with negative outcomes of the cessation attempt.

Two items assessed social influence measuring smoking status of the partner (yes, no, not applicable) and residential children (all, the majority, half, a minority, none, not applicable). The items were each transformed into 3 category-scales for inclusion in analyses: (-1 non-smoking, 0 not applicable, 1 smoking).

Intention to quit smoking was assessed with one item by asking respondents to state within what period they intended to quit smoking on a 7-point scale (within 1 month, within 6 months, within 1 year, within 5 years, at some point but not within 5 years, never quit but smoke less or use tobacco products with lower nicotine and tar levels and never quit or smoke less or use tobacco products with lower nicotine and tar levels). For analysing purposes, the 7-point scale was reduced to three categories of the stages of change: pre-contemplator (not to quit within 6 months), contemplator (to quit within 6 months) and preparer (to quit within 1 month).

Action plans were assessed by 5 items asking respondents to indicate whether they would use a specific action plan if they attempted to quit smoking (quit immediately, remove all smoking materials from the home, inform people around me, seeking cessation aids and prospecting a reward for myself).

Highest accomplished educational level was assessed at follow-up using one item on a 7-point scale (primary school, basic vocational school, secondary vocational school, high school, higher vocational school, college, university). 


\section{Analyses}

Data was analysed using SPPS 11.0. In order to stay as close as possible to the collected data, missing values were not substituted; sample size will therefore be reported for each analysis separately.

Factor analyses showed 3 distinct factors within attitude: pros and cognitive cons of quitting smoking (5 items, $\alpha=.83$ and 11 items, $\alpha=.55$, respectively) and affective cons of smoking ( 3 items, $\alpha=.76$ ). Four self-efficacy factors were determined: in emotional (4 items, $\alpha=.86$ ), habitual (4 items, $\alpha=.88$ ), social ( 4 items, $\alpha=.86$ ) and negative outcome ( 3 items, $\alpha=.65)$ situations. From these factors, mean sum scores were calculated. To limit the number of missing values, computation allowed for a single missing value within each sum score.

Respondents were assigned to groups according to the recruitment method used to include them; active recruitment in community pharmacy setting (AP), passive recruitment in community pharmacy setting (PP) and passive recruitment in general practice setting (PG). Differences in baseline characteristics were tested by means of univariate multinomial logistic regression analyses. This statistical test allows for a categorical variable (nominal or ordinal) as outcome variable and therefore dramatically reduces the number of tests conducted, which decreases the chance of type 1 error. PP served as reference category, which was compared to characteristics from AP and PG respondents, because this facilitates conclusions about the difference between active and passive method (in pharmacies) and the difference between the pharmacy and the general practice settings (for passive recruitment).

Univariate multinomial logistic regression was also used to test the acquired samples' representativeness concerning gender, age, education, stage of change and number of cigarettes smoked per day were compared to those of a cohort of Dutch smokers. This is part of a continuous study regarding smoking habits among the Dutch population aged 15 years and above (a random sample of 2000 households, weighed for gender and age), under the authority of the Dutch Foundation on Smoking and Health

(Continuous Survey of Smoking Habits (CSSH), unpublished data). Only smoking 
respondents were included in the analyses. The cohort sample served as reference category. As age was recorded in categories (e.g. 25-34 years), recruited respondents' age was recoded accordingly.

Differences in recruitment rate were calculated using one-way ANOVA with Tukey post-hoc tests.

\section{Results}

Recruited samples

A total of 1210 respondents were included in the analyses: 762 from active recruitment pharmacies, 163 respondents from passive recruitment pharmacies and 285 from passive recruitment general practices. Slightly more respondents were female $(n=680,56 \%)$ and mean age was 44 (range 14-79). The majority (62\%) indicated to have enjoyed secondary vocational training or higher. The sample included 432 (36\%) pre-contemplators, 311 (26\%) contemplators and $432(36 \%)$ preparers. Respondents showed a mild smoking addiction and mean number of cessation attempts in the past was 2.49. The mean number smoked daily was 22 cigarettes. A smoking partner was reported by 450 (37\%) respondents, 134 respondents (11\%) indicated having smoking residential children. Table 6.1 summarizes mean scores on all determinants for each sample separately.

\section{Cohort sample}

The cohort sample included 18212 respondents, of which 5499 (30\%) were current smokers. More male than female smokers were included in the sample $(55 \%$ and $45 \%$, respectively) and $70 \%(n=3843)$ of respondents were aged between 25 and 55 years. More than half $(57 \%)$ of the smoking respondents received secondary vocational training or higher ( $n=3112)$. The sample included 1827 (33\%) pre-contemplators, 492 (9\%) contemplators and 360 (7\%) preparers (51\% missing). Respondents smoked an average of 15 cigarettes per day. 
Table 6. 1.

Respondents' baseline characteristics for recruitment method separately.

\begin{tabular}{|c|c|c|c|c|c|}
\hline & Range & $\begin{array}{r}\text { Missing values } \\
(n)\end{array}$ & $\mathrm{AP}$ & PP & PG \\
\hline Sample size $(n)$ & - & - & 762 & 163 & 285 \\
\hline Recruitment sites (n) & - & - & 65 & 15 & 75 \\
\hline Receiving tailored advice & $0 / 1$ & - & $48 \%$ & $52 \%$ & $48 \%$ \\
\hline Female gender & $0 / 1$ & 34 & $56 \%$ & $59 \%$ & $62 \%$ \\
\hline Age (mean) & $14 / 79$ & 11 & 45 & 43 & 42 \\
\hline Education & $1 / 6$ & 300 & & & \\
\hline $\begin{array}{l}\text { - Primary school/basic vocational } \\
\text { school }\end{array}$ & & & $25 \%$ & $20 \%$ & $33 \%$ \\
\hline $\begin{array}{l}\text { - Secondary vocational school/high } \\
\text { school degree }\end{array}$ & & & $49 \%$ & $48 \%$ & $46 \%$ \\
\hline $\begin{array}{l}\text { - Higher vocational school/college } \\
\text { degree/ university degree }\end{array}$ & & & $27 \%$ & $32 \%$ & $21 \%$ \\
\hline Addiction level (mean) & $1 / 6$ & 22 & 3.22 & 3.39 & 3.44 \\
\hline $\begin{array}{l}\text { Previous cessation attempts } \\
\text { (mean) }\end{array}$ & $0 / 30$ & 44 & 2 & 3 & 2 \\
\hline Cardiovascular diseases & $0 / 1$ & 26 & $10 \%$ & $9 \%$ & $7 \%$ \\
\hline Respiratory diseases & $0 / 1$ & 27 & $22 \%$ & $15 \%$ & $20 \%$ \\
\hline Pros (mean) & $0 / 3$ & 8 & 1.16 & 1.23 & 1.14 \\
\hline Cognitive cons of cessation (mean) & $0 / 3$ & 19 & 1.85 & 2.05 & 1.86 \\
\hline Affective cons of smoking (mean) & $0 / 3$ & 11 & 1.17 & 1.28 & 1.24 \\
\hline Smoking partner & $0 / 1$ & 27 & $38 \%$ & $34 \%$ & $40 \%$ \\
\hline Smoking residential children & $0 / 1$ & 30 & $12 \%$ & $8 \%$ & $11 \%$ \\
\hline Social self-efficacy (mean) & $-2 / 2$ & 37 & -0.07 & -0.11 & -0.02 \\
\hline Habitual self-efficacy (mean) & $-2 / 2$ & 33 & -0.10 & -0.27 & -0.19 \\
\hline Emotional self-efficacy (mean) & $-2 / 2$ & 32 & -0.53 & -0.51 & -0.51 \\
\hline $\begin{array}{l}\text { Negative outcome self-efficacy } \\
\text { (mean) }\end{array}$ & $-2 / 2$ & 32 & -0.04 & -0.05 & -0.13 \\
\hline Intention to quit smoking & $1 / 3$ & 35 & & & \\
\hline - Pre-contemplation & & & $41 \%$ & $30 \%$ & $30 \%$ \\
\hline - Contemplation & & & $23 \%$ & $35 \%$ & $31 \%$ \\
\hline - Preparation & & & $37 \%$ & $35 \%$ & $38 \%$ \\
\hline Action plan: quit immediately & $0 / 1$ & 13 & $71 \%$ & $84 \%$ & $73 \%$ \\
\hline Action plan: remove materials & $0 / 1$ & 14 & $55 \%$ & $70 \%$ & $62 \%$ \\
\hline Action plan: inform people & $0 / 1$ & 13 & $47 \%$ & $54 \%$ & $57 \%$ \\
\hline Action plan: seek cessation aids & $0 / 1$ & 12 & $61 \%$ & $69 \%$ & $72 \%$ \\
\hline Action plan: prospect award & $0 / 1$ & 12 & $31 \%$ & $37 \%$ & $41 \%$ \\
\hline
\end{tabular}




\section{Recruitment rate}

Active recruiting pharmacies $(n=50)$ yielded an average of 15 respondents per pharmacy (range 2-51), passive recruitment pharmacies $(n=15)$ recruited a mean of 11 respondents per pharmacy (range 2-36) and passive recruitment general practices $(n=75)$ resulted in an average of 4 respondents per practice (range $0-11$ ) to be included in the study. These differences in recruitment rates proved to be significant between passive recruitment general practices and both passive and active recruitment pharmacies $(\mathrm{F}=33.8, \mathrm{p}<.01)$; general practices recruited significantly less respondents than both recruitment methods applied in community pharmacy setting.

\section{Differences between recruitment methods and settings}

Differences between samples acquired by the three recruitment methods are summarized in table 6.2.

Comparing active with passive recruiting in pharmacies, AP recruitment resulted in a sample with significantly more pre-contemplators and preparers. Also, the AP recruitment sample showed trends towards a higher age and more respondents reporting respiratory diseases compared to PP recruitment.

Furthermore, AP recruited respondents reported fewer cessation attempts in the past and saw less cons of smoking. A trend towards an increased level of self-efficacy in habitual situations was also observed for the AP recruitment sample. The intention to use specific action plans was lower in the AP recruitment sample for quitting immediately, removing smoking materials from the home, seeking cessation aids and informing people around them (trend).

Compared to PP recruitment, PG recruitment seemed to have resulted in a sample with a significantly lower education level, respondents had made fewer cessation attempts in the past and indicated to see less cons of smoking. Additionally, PG recruitment resulted in a sample with a lower intention to use the action plans to quit immediately and remove smoking materials (trend). 
Table 6.2.

Odds ratios calculated by means of multinomial logistic regression, comparing passive recruitment pharmacies $(P P)$ with active recruitment pharmacies $(A P)$ and passive recruitment general practices $(P G)$.

\begin{tabular}{|c|c|c|c|c|c|c|c|}
\hline & \multirow{3}{*}{$\begin{array}{c}\text { Sample }^{1} \\
(n)\end{array}$} & \multicolumn{3}{|c|}{$\mathrm{AP}$} & \multicolumn{3}{|c|}{ PG } \\
\hline & & \multirow[t]{2}{*}{ OR } & \multicolumn{2}{|c|}{$95 \% \mathrm{CI}$} & \multirow[t]{2}{*}{ OR } & \multicolumn{2}{|c|}{$95 \% \mathrm{CI}$} \\
\hline & & & Lower & Upper & & Lower & Upper \\
\hline Gender & 1176 & 0.90 & 0.64 & 1.27 & 1.13 & 0.76 & 1.68 \\
\hline Age & 1200 & $1.17 \ddagger$ & 0.98 & 1.38 & 0.92 & 0.76 & 1.12 \\
\hline Education & 910 & 0.89 & 0.72 & 1.09 & $0.70^{* *}$ & 0.55 & 0.88 \\
\hline Addiction level & 1188 & 0.90 & 0.76 & 1.07 & 1.04 & 0.85 & 1.26 \\
\hline Previous cessation attempts & 1166 & $0.84^{*}$ & 0.73 & 0.96 & $0.84^{*}$ & 0.71 & 0.99 \\
\hline Cardiovascular diseases & 1184 & 1.18 & 0.65 & 2.14 & 0.79 & 0.39 & 1.62 \\
\hline Respiratory diseases & 1183 & $1.57 \ddagger$ & 0.99 & 2.51 & 1.40 & 0.83 & 2.37 \\
\hline Pre-contemplation ${ }^{2}$ & 1175 & $2.15^{* *}$ & 1.39 & 3.31 & 1.15 & 0.71 & 1.88 \\
\hline Preparation $^{2}$ & 1175 & $1.61^{*}$ & 1.06 & 2.44 & 1.23 & 0.77 & 1.95 \\
\hline Pros of cessation & 1202 & 0.89 & 0.75 & 1.05 & 0.86 & 0.70 & 1.04 \\
\hline Cognitive cons of cessation & 1191 & $0.71^{* *}$ & 0.59 & 0.85 & $0.73^{* *}$ & 0.59 & 0.89 \\
\hline Affective cons of smoking & 1199 & 0.88 & 0.75 & 1.04 & 0.96 & 0.79 & 1.16 \\
\hline Smoking partner & 1184 & 1.09 & 0.86 & 1.37 & 0.95 & 0.73 & 1.24 \\
\hline Smoking children & 1180 & 0.98 & 0.84 & 1.13 & 0.93 & 0.79 & 1.10 \\
\hline Social self-efficacy & 1173 & 1.04 & 0.88 & 1.23 & 1.08 & 0.89 & 1.32 \\
\hline Habitual self-efficacy & 1177 & $1.15 \ddagger$ & 0.98 & 1.37 & 1.07 & 0.88 & 1.30 \\
\hline Emotional self-efficacy & 1178 & 0.99 & 0.83 & 1.17 & 1.00 & 0.83 & 1.22 \\
\hline $\begin{array}{l}\text { Negative outcome self- } \\
\text { efficacy }\end{array}$ & 1178 & 1.01 & 0.86 & 1.19 & 0.92 & 0.76 & 1.12 \\
\hline $\begin{array}{l}\text { Action plan (AP): quit } \\
\text { immediately }\end{array}$ & 1197 & $0.48^{* *}$ & 0.31 & 0.75 & $0.53^{*}$ & 0.32 & 0.87 \\
\hline AP: remove materials & 1196 & $0.51^{* *}$ & 0.35 & 0.73 & $0.69 \ddagger$ & 0.46 & 1.05 \\
\hline AP: inform people & 1197 & $0.75 \ddagger$ & 0.53 & 1.05 & 1.15 & 0.78 & 1.69 \\
\hline AP: seek cessation aids & 1198 & $0.69^{*}$ & 0.48 & 1.00 & 1.13 & 0.74 & 1.72 \\
\hline AP: prospect reward & 1198 & 0.76 & 0.54 & 1.09 & 1.15 & 0.77 & 1.71 \\
\hline
\end{tabular}

Reference category is PP

${ }^{1}$ total sample $\mathrm{n}=1210$

2 reference category is contemplation

$\ddagger \mathrm{p}<.10,{ }^{*} \mathrm{p}<.05,{ }^{* *} \mathrm{p}<.01$. 
Effect of recruitment on the acquired samples

Differences between samples acquired by the three recruitment methods are summarized in table 6.3.

Table 6. 3.

Odds ratios calculated by means of multinomial logistic regression, a national cohort of Dutch smokers aged 15 years and above with the three recruited samples.

\begin{tabular}{|c|c|c|c|c|c|c|c|c|c|}
\hline & \multicolumn{3}{|c|}{$\mathrm{AP}$} & \multicolumn{3}{|c|}{ PP } & \multicolumn{3}{|c|}{ PG } \\
\hline & \multirow[t]{2}{*}{ OR } & \multicolumn{2}{|c|}{$95 \%$ CI } & \multirow[t]{2}{*}{ OR } & \multicolumn{2}{|c|}{$95 \%$ CI } & \multirow[t]{2}{*}{ OR } & \multicolumn{2}{|c|}{$95 \% \mathrm{CI}$} \\
\hline & & Lower & Upper & & Lower & Upper & & Lower & Upper \\
\hline Female gender & $1.57^{* * *}$ & 1.34 & 1.83 & $1.74^{* *}$ & 1.26 & 2.40 & $1.97^{* * *}$ & 1.54 & 2.52 \\
\hline $\begin{array}{l}\text { Age } \\
\text { (categorised) }\end{array}$ & $1.18^{* * *}$ & 1.12 & 1.25 & 1.06 & 0.95 & 1.18 & 1.03 & 0.95 & 1.12 \\
\hline Education & $1.09^{* *}$ & 1.03 & 1.15 & $1.18^{* *}$ & 1.04 & 1.35 & 0.92 & 0.84 & 1.00 \\
\hline $\begin{array}{l}\text { Number of } \\
\text { cigarettes } \\
\text { smoked per } \\
\text { day }\end{array}$ & $1.07^{* * *}$ & 1.06 & 1.08 & $1.07^{* * *}$ & 1.06 & 1.08 & $1.07^{* * *}$ & 1.05 & 1.08 \\
\hline $\begin{array}{l}\text { Pre- } \\
\text { contemplation }{ }^{1}\end{array}$ & $0.49^{* * *}$ & 0.39 & 0.60 & $0.23^{* * *}$ & 0.15 & 0.34 & $0.26^{* * *}$ & 0.19 & 0.36 \\
\hline Preparation $^{1}$ & $2.20^{* * *}$ & 1.74 & 2.79 & 1.37 & 0.92 & 2.03 & $1.67^{* *}$ & 1.23 & 2.29 \\
\hline
\end{tabular}

$\mathrm{AP}=$ active recruitment in pharmacy, $\mathrm{PP}=$ passive recruitment in pharmacy, $\mathrm{PG}=$ passive recruitment in general practice

${ }^{* *} \mathrm{p}<.01,{ }^{* * *} \mathrm{p}<.001$

${ }^{1}$ reference category is contemplation

All three recruited samples included more women, heavier smokers and less precontemplators than the cohort sample. Only the AP sample included older participants.

Both recruitment methods in community pharmacy setting resulted in a higher educated sample; AP and PG samples included more preparers, compared to the cohort.

\section{Discussion and Conclusion}

The study described in this report aimed to determine the effect of active versus passive recruitment of smoking adults in general practices and community pharmacies on the samples acquired by these recruitment methods. 


\section{Discussion}

Recruitment samples differed in education level, quitting history, stage of change, cognitive cons of smoking and the use of specific action plans when trying to quit. Trends indicated a difference in age, occurrence of respiratory diseases, habitual selfefficacy and a number of action plans. This confirms results from previous research that recruitment methods influence the sample acquired.

Both recruitment methods in community pharmacy setting yielded a significant higher recruitment rate than passive recruitment in general practice setting. This indicates that when recruiting in general practice, a lower yield must be expected. However, it could in part be caused by the pharmacy being a reasonably new recruitment setting; the novelty might have increased the response rate of both active and passive recruitment methods.

In community pharmacy setting, active recruitment resulted in more participants than passive recruitment, but not significantly so. This implies that in community pharmacy an active recruitment is not preferable over passive recruiting. As previous studies used methods differing from the ones used in this study, as well as target population and recruitment setting, it is difficult to adequately compare these results with previous studies conducted.

Actively recruited respondents saw less cons of smoking, reported fewer attempts to quit in the past and were less likely to intend to use action plans when quitting compared to passively recruited respondents in community pharmacy setting.

Additionally, more pre-contemplators were included in the actively recruited sample. This indicates that active recruitment in community pharmacy provides a sample of smokers usually more difficult to obtain in smoking cessation intervention studies, namely those smokers who do not want to quit, have not tried often in the past and are not making any plans concerning quitting. Additionally, they are not convinced of the disadvantages of their smoking habit. Active recruitment in community pharmacy setting therefore seems a plausible choice when trying to reach a smoking population less interested in quitting. 
Passively recruited respondents in general practice seemed to have a lower educational level than respondents passively recruited in community pharmacy. Unfortunately, this variable included a large number of missing values $(n=300,25 \%)$, as not all respondents were reached at follow-up (when this question was asked). It is therefore not possible to make firm judgements on the basis of the significant result found. It can be argued that respondents not reached at follow-up had a higher educational level, which coloured the results from the analyses. However, the sample of respondents included by general practice passive recruitment showed a smaller number of missing values on education than community pharmacy active and passive recruitment samples (passive general practice $16 \%$ missing, active community pharmacy $25 \%$ missing, passive community pharmacy $37 \%$ missing). When education is considered to be an indicator of socioeconomic status (SES), general practice might be preferred over community pharmacy when trying to recruit lower SES participants.

All three samples appeared to be significantly different from the cohort sample. The sample actively recruited in community pharmacy setting was the least representative of the three samples, with significant differences on all demographics assessed. The general practice setting was comparable with the cohort sample regarding age and education level. As respondents were recruited to participate in a smoking cessation intervention, it is not surprising that respondents were more motivated to quit, as a smoking cessation intervention would appeal more to smokers willing to change their behaviour than to smokers who do not want to change their smoking behaviour. Recruited respondents also smoked more cigarettes per day, this might be linked to cohort respondents more often reporting smoking no cigarettes a day than recruited respondents $(2.73 \%$ versus $0.08 \%$, respectively), indicating an occasional smoking pattern. Occasional smokers might consider themselves less of a smoker and the smoking behaviour less of a problem that needs to be dealt with. Previous studies have shown that women consult their general practitioner more often than men (Bayram et al. 2003; Rickards et al. 2004); they could therefore have been more exposed to the recruiting. When this assumption is continued into community pharmacy setting, it 
could partly explain the overrepresentation of female respondents in the recruited samples.

Main strengths of this study were the large sample sizes, the separation of recruitment method and setting, and the as yet unstudied comparison of general practice versus community pharmacy as recruitment setting. All comparisons included either recruitment method or setting as a constant factor. This facilitated the separate comparisons between different settings and different methods. Furthermore, the acquired samples were compared to a cohort of Dutch smokers, testing the recruited samples' deviation from the average group of smokers.

However, some limitations are also current. Firstly, active recruitment in general practice setting was not included in the study. As the community pharmacy recruitment was conducted before the general practice recruitment started, adjustments in design were possible. In community pharmacy, it proved to be difficult to maintain active recruiting during the entire recruitment period. It was therefore likely that it would also prove difficult in general practice. In addition, previous studies have shown difficulties in including general practices in active recruitment and subsequently acquiring a substantial number of recruited participants (Hetherton et al. 2004). Therefore, the recruitment design was adjusted and only passive recruitment took place in general practice. Secondly, a small number of respondents recruited were younger than the inclusion criterion set at 18 years or older $(n=13)$, but this is not likely to have skewed the data as an even distribution among the three recruitment methods can be observed (active community pharmacy $0.7 \%$ under-aged, passive general practice $1.4 \%$ under-aged and active community pharmacy $2.5 \%$ under-aged). Additionally, the cohort sample also included $2.9 \%$ under-aged respondents. Thirdly, response rate analyses could not be conducted, as the participant pool was not clearly defined and nonresponders' characteristics could therefore not be assessed either. Lastly, as it is not clear how many smokers have been exposed to either active or passive recruitment but decided against participation, response rates could not be calculated and nonresponders' characteristics were not assessed. Therefore, it is not possible to test whether 
the populations exposed to the different recruitment methods and settings significantly differed or that the willingness of smokers with specific characteristics (e.g. participants with a certain educational level) differed between the recruitment methods and settings. However, the issue of the samples' representability has been studied by comparing the samples to a representative cohort.

\section{Conclusion}

The results from the analyses described show that samples recruited by different methods and settings differ significantly on its characteristics. Although through general practice fewer smokers were recruited, this setting could be useful when trying to get access to smokers of a lower socio-economic class. A more active approach of recruitment in community pharmacy is not significantly more effective than passive recruitment. However, active recruitment resulted in significantly more precontemplators. Interaction between pharmacy staff and smokers might have helped to overcome initial resistance from pre-contemplators to participate in a smoking cessation study, as they did not have the intention to quit smoking in the near future. Although passive recruitment also stressed that participants did not have to quit smoking, no interpersonal interaction might have limited its effect on pre-contemplators.

As all recruited samples differed significantly from the cohort sample, it can be concluded that recruiting for a smoking cessation intervention in general practices and community pharmacies does not provide a cross-section of the Dutch smoking population. However, the general practice sample appeared to have most in common with the cohort.

\section{Practice Implications}

The results of this study can be used by researchers deciding on a recruitment strategy for their studies and practitioners for the recruitment for their interventions when contemplating on recruitment through primary care health professional setting. Recruitment through general practices seems to be to recruit lower socio-economic class 
smokers and appears to yield the sample most representative of the Dutch smoking population. Active recruitment in community pharmacy could be used to recruit precontemplating smokers. Researchers employing only limited or no eligibility criteria could benefit from passive recruitment in community pharmacy. Results show that recruiting for a smoking cessation intervention in general practices and community pharmacies does not provide a representation of the Dutch smoking population. 



\section{CHAPTER 7}

\section{Smoking and the $\varnothing$ pattern; predictors of transitions through the stages of change}

Abstract

Pre-contemplators, contemplators and preparers have previously been described by distinct scores on pros, social influence and self-efficacy; the $\varnothing$ pattern. The objective of this study was to replicate this pattern in a sample of adult smokers $(n=554)$, to longitudinally observe stage sequence and identify predictors for forward and backward stage transition. Three hypotheses were formulated 1) forward transition from precontemplation will be predicted by a higher perception of pros concerning smoking cessation, 2) backward transition from contemplation will be predicted by a lower perception of pros of quitting smoking and 3) forward transition from preparation will be predicted by a higher self- efficacy perception concerning smoking cessation. The $\varnothing$ pattern was replicated successfully. Smokers appeared to be more likely to transition to an adjacent stage than to skip a stage in the sequence. For prediction of stage transition, separate analyses were conducted for pre-contemplators, contemplators and preparers. Respondents transitioning forward were compared to respondents remaining in the same stage or transitioning backward and vice versa. Hypothesis one and three were confirmed. Additionally, self-efficacy predicted forward transition from precontemplation. Implications towards improving interventions and research concerning stage transition are discussed.

Published as: Hoving EF, Mudde AN, de Vries $H$ (2006). Smoking and the Ø pattern; predictors of transitions through the stages of change. Health Educ Res, 21(3), 305-314. 


\section{Introduction}

Behaviour change is recognised as a process rather than a static moment in time in which behaviour alternation occurs. The stages of change, as proposed by Prochaska and DiClemente in the Transtheoretical Model (Prochaska and DiClemente 1983), are a product of this line of thinking. According to the stages of change, smokers do not simply change into non-smokers overnight, but go through different stages of intention in order to reach behavioural change. These stages are based on the time indicated by the individual between the current moment and the intended behaviour change and are classified as pre-contemplation (do not wish to quit within six months), contemplation (wish to quit within six months) and preparation (wish to quit within one month and has seriously attempted to quit smoking in the last year). After the behavioural change has taken place (action), the changed behaviour can be continued (maintenance) or discontinued (relapse) (Prochaska et al. 1997).

These stages are incorporated in the I-Change Model (De Vries et al. 2003), the theoretical background of this study. This model, succeeding the ASE Model, is based on several cognitive models and its concepts have been used as a theoretical basis for a number of studies concerning behavioural change (Ausems et al. 2002; Bolman and de Vries 1998; De Nooijer et al. 2003). The motivational concepts of pros, social influence and self-efficacy are acknowledged to be of influence on the intention to change behaviour, the stages of change. These three core elements are in turn influenced by predisposing factors. The I-Change Model differs from the ASE Model in that it also takes awareness factors (knowledge, risk perception and cues to action) and information factors (the quality, channel and source of information concerning the behaviour change) into account. Studies on the characteristics of smokers concerning the motivational concepts across the stages of change within the context of the I-Change Model showed a specific pattern of pros, social influence and self-efficacy scores shaped as a $\varnothing$ (see Figure 7.1), demonstrating differences in scores on these three concepts across the stages. 


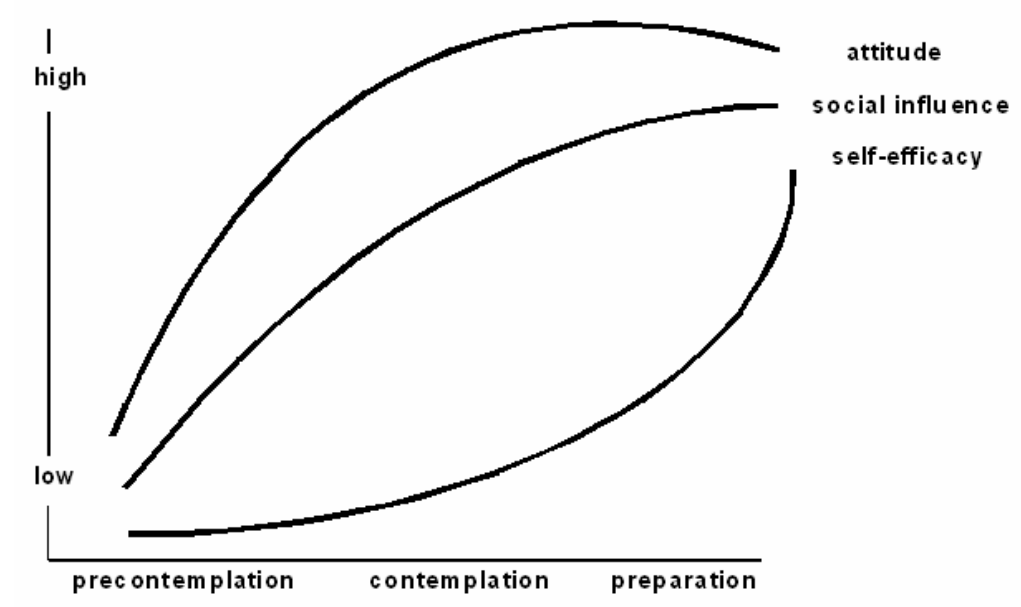

Fig 7.1. The $\varnothing$ Hypothesis.

De Vries and Backbier (1994) initially described the $\varnothing$ hypothesis in a population of Dutch adult smokers, where pre-contemplators perceived less advantages or pros of smoking cessation than contemplators and contemplators seemed to have a lower score on self-efficacy compared to those individuals in the action stage. In addition, perceived social support increased steadily across the stages towards action. Later studies confirmed these findings in samples of cross-sectional (De Vries et al. 1998) and longitudinal data (De Vries and Mudde 1998; De Vries et al. 2000) for smoking cessation. Results of these latter studies indicated that forward transition from pre-contemplation was predicted by seeing more pros of quitting smoking, whereas a higher self-efficacy score predicted forward transition from preparation to action. Backward transition from contemplation was predicted by perceiving less pros of quitting. However, the number of longitudinal studies on stage transition in a population of smokers is limited and results are not always conclusive. Additionally, not all studies have used the stage of change algorithm.

Although the stages of change have been widely applied in social science research, they have also received a fair amount of criticism. Main concern is that the 
stages of change might not be 'real' stages of which transition can be predicted by increases or decreases in cognitive concepts, but are merely pseudo-stages that are concealing a continuum of these concepts (Bandura 1997; Weinstein et al. 1998). This would imply no stage transition-specific predictors. Additionally, the time cut-off points between the stages are considered arbitrary (Sutton 2001). Weinstein (1998) suggests 4 types of research to test the validity of the stages of change. This study includes 3 of these suggested research methods: cross-sectional comparisons between characteristics of subjects in the different stages of change (by replicating the $\varnothing$ pattern), examination of stage sequences over time and longitudinal prediction of backward and forward stage transitions from the stages pre-contemplation, contemplation and preparation in a sample of adult smokers. The fourth suggested test, experimental studies of matched and mismatched interventions, was not within the scope of the present study. Three hypotheses regarding stage transitions were formulated, based on results from a previous study (De Vries and Mudde 1998): 1) forward transition from precontemplation will be predicted by a higher perception of pros concerning smoking cessation, 2) backward transition from contemplation will be predicted by a lower perception of pros of quitting smoking and 3) forward transition from preparation will be predicted by a higher self-efficacy perception concerning smoking cessation As the $\varnothing$ pattern describes the influence of social influence as gradually increasing over the stages (De Vries and Mudde 1998) and earlier studies did not found a significant predictive value concerning stage transition, no specific hypothesis was formulated. Additionally, the influence of cons of smoking cessation was assessed.

\section{Methods}

Design

Data was collected in order to test the effectiveness of a computer-generated tailored smoking cessation expert system in Dutch community pharmacy setting. Respondents were randomly assigned to either the experimental group (receiving a computergenerated personalised advice letter) or the control group (receiving only a thank you letter, no additional information). As respondents from both groups were included in 
this study, all analyses were controlled for condition. Three and twelve months after baseline measurement, all respondents were contacted by telephone for follow-up. Only respondents with a valid measurement on baseline and both follow-ups were included in the analyses; respondents whose gender was unknown and cases with $>10 \%$ missing values at one or more measuring points were excluded.

\section{Procedure}

Respondents were recruited for a randomised control trial via 65 community pharmacies throughout the Netherlands, offering them the chance to receive computer-generated personalised smoking cessation advice. Eligible participants were informed that one in two smokers would receive this advice. Smokers who had smoked in the last seven days and were over 18 years of age were eligible for participation. Participants were asked to fill in a baseline questionnaire. A total of 915 smokers filled in this questionnaire and returned it.

\section{Intervention}

The expert system collects information on a smoker by means of a baseline written questionnaire (content is described below). This questionnaire was experimentally tested in a previous study (Dijkstra et al. 1996; Dijkstra et al. 1998b) and is now serving as the screening instrument for a computer-generated tailored smoking cessation programme distributed nationwide by the Dutch Foundation on Smoking and Health (STIVORO for a smokefree future). Respondents send their questionnaires to a central processing centre, the answers given are used to generate a personalised letter, which the respondents receive at their home address. The content of the letter is stage-tailored and provides feedback on a smoker's personal attitudes towards smoking and cessation, selfefficacy in various situations and actions plans when trying to quit.

\section{Baseline questionnaire}

A written baseline questionnaire consisting of 54 questions was used to collect demographic data (gender, age, addiction level, the occurrence of cardiovascular and 
respiratory diseases), number of previous quit attempts, intention to quit smoking (stage of change) and the concepts of attitude, social influence and self-efficacy.

Addiction level was measured with three items, using the abbreviated Fagerström scale (Heatherton et al. 1991); the type of tobacco product smoked, the number of tobacco products smoked per day and time passed before smoking after getting out of bed, from which a sum score was calculated (range 0-6).

The occurrence of cardiovascular and respiratory diseases was measured by one dichotomous item each.

Previous quit attempts were assessed by asking respondents how often they had attempted to quit smoking in the past.

Questions concerning attitude and self-efficacy were formulated as statements; respondents could indicate on a 5-point scale to what extent they agreed with the statement given.

Attitude was assessed using 16 items. Factor analysis revealed two attitude factors: the advantages of smoking cessation/pros (e.g. improving own health and that of people around the smoker, financial saving; 11 items, $\alpha=.83$ ) and disadvantages of smoking cessation/cons (e.g. gaining weight, boredom, symptoms of withdrawal; 5 items, $\alpha=.52)$.

Self-efficacy was measured by 15 items on a 5-point scale; respondents were asked to indicate if they would be able to refrain from smoking in emotional situations (e.g. when being angry, when being stressed; 4 items), habitual situations (e.g. after dinner, on a break, drinking coffee or tea; 4 items), in social situations (when being offered a cigarette, when seeing someone else enjoying smoking; 4 items) and when being confronted with negative outcomes of the cessation attempt (e.g. when craving for a cigarette, when noticing weight gain; 3 items). All items were combined into one selfefficacy factor $(\alpha=.92)$.

Two items measured social influence (modelling) assessing smoking status of partner and children in residence.

Intention to quit smoking was assessed by asking respondents to state on a 7point scale when they intended to quit smoking. Respondents were then classified as 
preparers (intention to quit within a month), contemplators (intention to quit within the next six months) or pre-contemplators (no intention to quit within the next six months).

\section{Follow-ups}

In both telephonic follow-up measurements, respondents' stage of change (precontemplation, contemplation, preparation) and current smoking status (whether or not the respondent had been smoking in the past 7 days) were measured, as well as quitting behaviour between baseline and follow-up; respondents were asked whether they have attempted to quit smoking during the study period. Educational level and year of birth were also assessed.

Analyses

Smokers were grouped according to their self-reported intention to quit smoking (stage of change) at all three measuring points. At baseline, respondents were identified as preparers, contemplators or pre-contemplators. Classification for the follow-ups was conducted accordingly; respondents who were not smoking at follow-up were then classified as actors. By comparing the stage of change from the follow-up to the stage of change at baseline, it was determined whether a respondent had moved forward (a positive shift towards the action stage), backward (a negative shift towards the precontemplation stage) or had not moved.

First, attrition analyses were conducted in order to determine whether respondents lost to follow-up were significantly different from those who were available for follow-ups.

Secondly, z-scores were calculated for items and factors concerning attitude, social influence and self-efficacy. The calculation of z-scores was performed for the total sample and for respondents in preparation, contemplation and pre-contemplation separately.

Thirdly, univariate GLM with Sidak contrasts were calculated from crosssectional data of the baseline measurement between preparers, contemplators and precontemplators, in order to cross-sectionally replicate the $\varnothing$ pattern. 
Fourthly, in order to determine predictors of both forward (positive) and backward (negative) stage transitions from preparation, contemplation and pre-contemplation, three and 12 months after baseline, stepwise logistic regression analyses were conducted.

Respondents who had moved forward were compared with respondents who had remained at the same stage or had moved backward. The same procedure was followed for backward transitions from each stage: respondents who had moved backward were compared with those who had remained in the same stage or had moved forward. Items and factors were included in the regression in two blocks: 1) demographic variables and 2) attitudes, social influence and self-efficacy. To ascertain that only significant demographic variables were entered into the analysis, the forward method was used on both blocks. The exclusion criterion was increased to 0.20 , as recommended by Hosmer and Lemeshow (1989) in order to compensate for stepwise analysis. To neutralise any effect the intervention might have had on transitions through the stages, the concept of condition remained in the analyses as a covariate, regardless of its significance level. As motivational determinants were not assessed at either follow-up, analyses comparing transition between $1^{\text {st }}$ and $2^{\text {nd }}$ follow-up could not be conducted.

\section{Results}

Sample

Analyses included a total of 554 respondents. The sample included slightly more females (56\%) and the mean age was 46 years. Fifty-eight (11\%) respondents indicated that they suffered from cardiovascular diseases, chronic pulmonary diseases were reported by 113 (20\%) respondents. Three respondents were pregnant when filling out the questionnaire. A minority only received basic education $(n=35,6 \%)$, one fifth $(n=116,21 \%)$ attended only high school, 100 (18.1\%) respondents received primary vocational training, 156 $(28.2 \%)$ respondents received secondary vocational training and one quarter $(n=147$, $26.5 \%$ ) attended higher vocational training or university. Most respondents (66\%) smoked manufactured cigarettes, 49\% smoked hand rolled cigarettes and $4 \%$ smoked cigars (some respondents smoked more than one type of tobacco). Only three respondents smoked pipe tobacco. Mean amount of tobacco product smoked per day 
was 22. Mean amount of previous cessation attempts was three, the average respondent had a mild nicotine addiction level (3.29). At baseline, 210 (38\%) respondents were classified as pre-contemplators, $144(26 \%)$ respondents were contemplators and 200 $(36 \%)$ respondents were identified as preparers.

Among respondents in the experimental group $(n=276) 55 \%$ was female, compared to $57 \%$ in the control condition $(n=278)$. In both conditions, mean age was 46 years. In the experimental condition $7 \%$ received basic education, $21 \%$ attended only high school, 17\% received primary vocational training, 30\% received secondary vocational training and $26 \%$ attended higher vocational training or university. For the control condition these percentages are 6, 21, 19, 27 and 27, respectively. In the experimental group, $40 \%$ was classified as pre-contemplator at baseline compared to $36 \%$ in the control condition; percentages for contemplation and preparation were $24 \%$ and $36 \%$ in the experimental group and $28 \%$ and $36 \%$ in the control group, respectively. In both groups, the average respondent had a mild nicotine addiction level.

Attrition analyses revealed that respondents included in the analyses were significantly older $(\mathrm{OR}=1.02, \mathrm{p}<.01)$ than respondents not included in the analyses $(n=371)$. Collinearity diagnostics revealed no existing multicollinearity or singularity.

Characteristics of pre-contemplators, contemplators and preparers; the Ø pattern

Pre-contemplators (PC) and preparers (PR) appeared to differ significantly concerning the number of previous cessation attempts $(\mathrm{PC}<\mathrm{PR})$, educational level $(\mathrm{PC}>\mathrm{PR})$ and the occurrence of cardiovascular diseases (PC $<$ PR). Means for z-scores of attitudes, social influence and self-efficacy, adjusted for these variables, are shown in table 7.1. Figure 7.2 shows the cross-sectional curve of attitudes, social influence and self-efficacy across the stages of pre-contemplation, contemplation and preparation in this sample. 
Table 7.1.

Differences at baseline between stages concerning z-scores of attitudes, social influence and self-efficacy.

\begin{tabular}{|c|c|c|c|c|}
\hline & Pre-contemplation & Contemplation & Preparation & \\
\hline Pros & -0.28 & 0.11 & 0.22 & $\mathrm{PC}<\mathrm{C}, \mathrm{PR}$ \\
\hline Cons & 0.00 & 0.11 & -0.15 & Ns \\
\hline $\begin{array}{l}\text { Smoking } \\
\text { partner }\end{array}$ & 0.06 & -0.01 & -0.02 & Ns \\
\hline $\begin{array}{l}\text { Smoking } \\
\text { residential } \\
\text { children }\end{array}$ & 0.01 & 0.09 & -0.17 & Ns \\
\hline Self-efficacy & -0.22 & -0.14 & 0.30 & $\mathrm{PC}, \mathrm{C}<\mathrm{PR}$ \\
\hline
\end{tabular}

Means are adjusted for condition

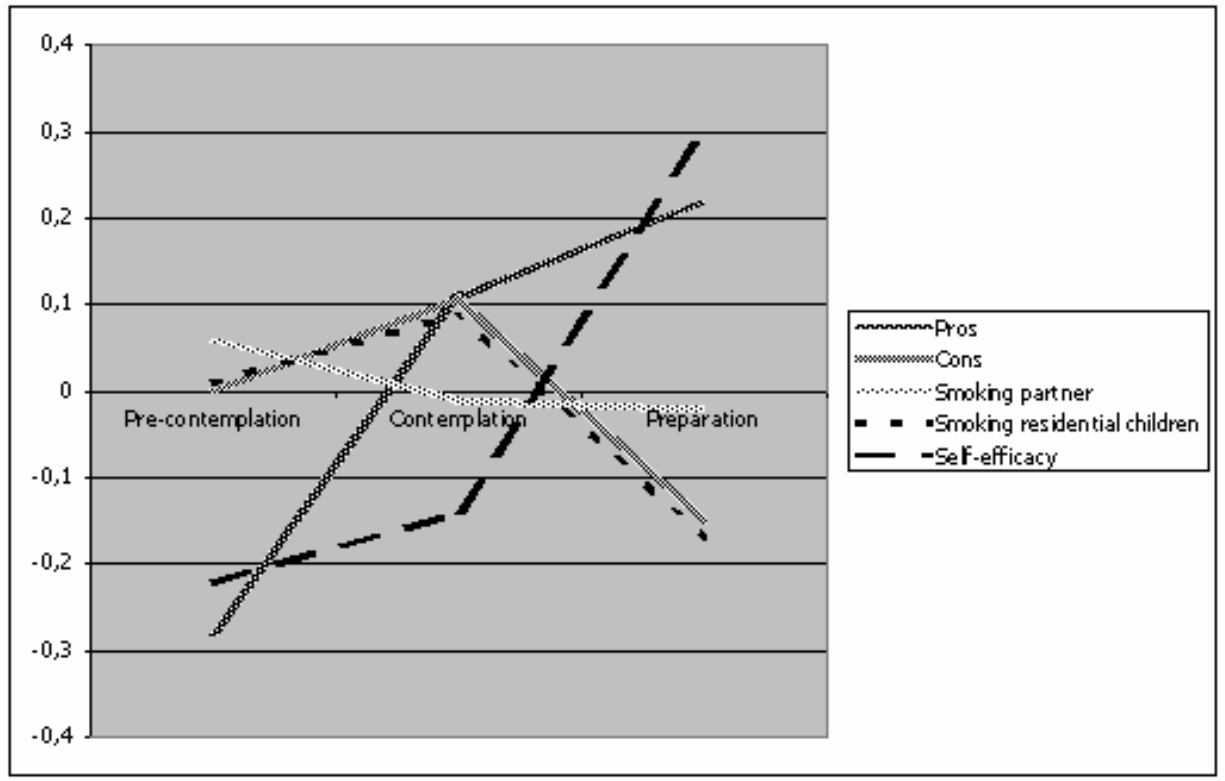

Fig 7.2. Distribution of z-scores of attitudes, social influence and self-efficacy over the stages of change.

Pre-contemplators saw significantly less pros of quitting smoking compared to contemplators and preparers. Contemplators saw significantly more cons of quitting 
than preparers, but pre-contemplators, contemplators and preparers did not differ significantly on the cons of smoking. Having a smoking partner or residential children smoking was not characteristic for any of the three stages. Preparers expressed a higher self-efficacy when trying to refrain from smoking than both contemplators and precontemplators did.

Predictors of stage transition 3 months after baseline

After three months, 246 (44\%) respondents did not move from one stage to another, 108 (19\%) respondents moved backward and 200 (36\%) respondents moved forward through the stages. A complete summary can be found in table 7.2. Of the smokers transitioning forward from their baseline stage, most transitioned to the adjacent stage (for instance, more pre-contemplators ended up in contemplation than in preparation or action). Due to limited sample size, we could not test these differences.

Table 7.2.

Stage transitions between baseline (TO) and $1^{\text {st }}$ follow-up.

\begin{tabular}{l|cccc}
\hline & $\begin{array}{c}\text { Pre-contemplation } \\
\text { at follow-up } \\
(n=158,29 \%)\end{array}$ & $\begin{array}{c}\text { Contemplation } \\
\text { at follow-up } \\
(n=171,31 \%)\end{array}$ & $\begin{array}{c}\text { Preparation } \\
\text { at follow-up } \\
(n=172,31 \%)\end{array}$ & $\begin{array}{c}\text { Action at } \\
\text { follow-up } \\
(n=53,10 \%)\end{array}$ \\
\hline $\begin{array}{l}\text { Pre- } \\
\text { contemplation } \\
\text { at baseline } \\
(n=210,38 \%)\end{array}$ & $\mathbf{1 0 0 ( 4 8 \% )}$ & $65(31 \%)$ & $27(13 \%)$ & $18(9 \%)$ \\
$\begin{array}{l}\text { Contemplation } \\
\text { at baseline } \\
(n=144,26 \%)\end{array}$ & $23(16 \%)$ & $56(39 \%)$ & $55(38 \%)$ & $10(7 \%)$ \\
$\begin{array}{l}\text { Preparation at } \\
\text { baseline } \\
(n=200,36 \%)\end{array}$ & $35(18 \%)$ & $50(25 \%)$ & $\mathbf{9 0}(\mathbf{4 5 \% )}$ & $25(13 \%)$ \\
\hline
\end{tabular}

Values on the diagonal indicate respondents who have not moved from one stage to another, values under the diagonal indicate respondents who moved backward and values above the diagonal indicate respondents who moved forward.

Smokers experiencing a forward transition from pre-contemplation to the contemplation, preparation or action stage perceived significantly more pros of quitting smoking than pre-contemplators who did not move through the stages (OR 1.40, 1.05- 
$.86, \mathrm{p}<.05)$. Having attempted to quit more often in the past showed a trend towards forward transition from pre-contemplation ( $O R$ 1.13, 1.00-1.28, $\mathrm{p}=.05$ ). Respondents moving forward from contemplation to the preparation or action stage had suffered more often from chronic pulmonary diseases (OR 2.76, 1.21-6.29, $\mathrm{p}<.05$ ) and were more confident to resist smoking (OR 1.47, 1.04-2.09, $\mathrm{p}<.05)$ than those who did not. Smokers experiencing a backward transition from contemplation to the pre-contemplation stage were more likely to be male (OR 0.3, 0.12-0.77, $\mathrm{p}<.05$ ). Forward transition from preparation after three months could not be predicted by the variables entered into the analysis. Backward transition from preparation to contemplation or pre-contemplation was predicted by a smaller amount of previous cessation attempts $(0.84,0.74-0.96, \mathrm{p}<.01)$.

\section{Predictors of stage transition 12 months after baseline}

When comparing baseline data with that of the second follow-up 12 months later, 208 $(38 \%)$ respondents did not move from one stage to another, 173 (31\%) respondents moved backward and $173(31 \%)$ respondents moved forward through the stages. A complete summary can be found in table 7.3. Of the smokers transitioning forward from their baseline stage, most transitioned to the adjacent stage. Due to limited sample size, we could not test these differences.

Respondents moving forward from pre-contemplation had experienced more previous cessation attempts in the past (OR 1.18, 1.04-1.35, $\mathrm{p}<.05$ ), perceived more pros of smoking cessation (OR 1.55, 1.15-2.10, p<.01) and showed a higher self-efficacy rate to be able to refrain from smoking once quitted (OR1.48, 1.10-1.99, p<.05). Smokers moving forward from contemplation could be predicted by the occurrence of cardiovascular diseases (OR 2.94, 1.06-8.19, $\mathrm{p}<.05)$; backward transition could not be predicted by the variables entered in the analyses. Smokers progressing to the action stage from preparation were more confident they would be able to refrain from smoking when trying to quit $(\mathrm{OR} 1.48,1.01-2.17, \mathrm{p}<.05)$ than smokers who did not progress forward from preparation. Backward transition from preparation after 12 months was predicted by a smaller number of previous cessation attempts (OR 0.89, 0.80-0.98, p<.05). 
Table 7.3.

Stage transitions between baseline (T0) and $2^{\text {nd }}$ follow-up.

\begin{tabular}{l|cccc}
\hline & $\begin{array}{c}\text { Pre-contemplation } \\
\text { at follow-up } \\
(n=234,42 \%)\end{array}$ & $\begin{array}{c}\text { Contemplation } \\
\text { at follow-up } \\
(n=149,27 \%)\end{array}$ & $\begin{array}{c}\text { Preparation } \\
\text { at follow-up } \\
(n=87,16 \%)\end{array}$ & $\begin{array}{c}\text { Action } \\
\text { at follow- } \\
\text { up } \\
(n=84, \\
15 \%)\end{array}$ \\
\hline $\begin{array}{l}\text { Pre-contemplation at } \\
\text { baseline } \\
(n=210,38 \%)\end{array}$ & $\mathbf{1 1 5 ( 5 5 \% )}$ & $41(20 \%)$ & $21(10 \%)$ & $33(16 \%)$ \\
$\begin{array}{l}\text { Contemplation at } \\
\text { baseline } \\
(n=144,26 \%)\end{array}$ & $47(33 \%)$ & $54 \mathbf{( 3 8 \% )}$ & $27(19 \%)$ & $16(11 \%)$ \\
$\begin{array}{l}\text { Preparation at } \\
\text { baseline } \\
(n=200,36 \%)\end{array}$ & $72(36 \%)$ & & & $35(18 \%)$ \\
\hline
\end{tabular}

Values on the diagonal indicate respondents who have not moved from one stage to another, values under the diagonal indicate respondents who moved backward and values above the diagonal indicate respondents who moved forward.

\section{Discussion}

Summary of the findings

In this study, three of the four research types suggested by Weinstein (1998) have been incorporated; cross-sectional comparisons between stages, stage sequencing and longitudinal prediction of stage transitions.

The first goal of this study was to compare stages with respect to cross-sectional differences in cognitive factors by replicating the $\varnothing$ pattern in a sample of Dutch adult smokers. We were successful in replicating this pattern; our results show that precontemplators perceived significantly less pros of quitting smoking whereas preparers showed higher self-efficacy to refrain from smoking once quitted.

The second goal was to examine stage sequencing. Smokers appeared to be more likely to transition to an adjacent stage than to skip a stage in the sequence. Due to limited sample size, we were not able to test the differences found.

The third goal was to examine possible predictors of stage transition over periods of three and 12 months respectively. For this purpose, three hypotheses were formulated. Concerning the first hypothesis (forward transition from pre-contemplation can be predicted by a higher perception of pros concerning smoking cessation), forward 
transition from pre-contemplation was predicted by perceiving more pros of quitting at baseline at both three and 12 months follow-up. No evidence was found to support the second hypothesis (backward transition from contemplation will be predicted by a lower perception of pros of smoking cessation). The third hypothesis (forward transition from preparation will be predicted by a higher self-efficacy perception concerning smoking cessation) was also confirmed; preparers who had moved forward to action after 12 months displayed more confidence to be able to refrain from smoking than those that moved backward or stayed in preparation.

Previous research shows some similarities with results found in this study, as well as some differences. Perceiving more pros predicting forward transition from precontemplation was found by several other recent studies concerning smoking and fruit and vegetable intake (De Vet et al. 2005; De Vries and Mudde 1998; Dijkstra et al. 2003). A higher self-efficacy predicting forward transition from preparation is also a common find. However, twelve months after baseline, self-efficacy also predicted a forward transition from pre-contemplation and contemplation. This was not expected, as selfefficacy is thought to play a role in later stages. As pre-contemplators at baseline who had remained in this stage at follow-up were compared with pre-contemplators moving forward to any stage (also preparation and action), this might have influenced this finding. However, other studies using a similar classification did not find self-efficacy to be predictive of forward transition from pre-contemplation (De Vet et al. 2005; De Vries and Mudde 1998).

Although some studies (De Vries and Mudde 1998) found that perceiving less pros predicted backward stage transition from the contemplation stage, Dijkstra and colleagues (2003) did not find predictors for this transition. Velicer et al. (1999) described contemplation as an unstable stage; contemplators are in conflict concerning their smoking behaviour, but have not yet made the decision to either prepare for a quit attempt (preparer) or to remain a smoker for the time being (pre-contemplator). They may have thought about the benefits of cessation, but are not yet sure that they will be able to quit themselves. This is confirmed by the fact that perceiving more pros predicted 
forward transition from pre-contemplation and a higher self-efficacy score predicted forward transition from contemplation.

Some studies (De Vries and Mudde 1998; Velicer et al. 1999) have suggested that perceiving less cons of smoking cessation may predict transition from precontemplation, but no predictive role was found for cons in this study for transition from any of the stages.

\section{Limitations}

This study has some limitations. Respondents included in this study were participating in a smoking cessation intervention; they may have been more motivated to make a forward transition through the stages in comparison with other smokers. However, during recruitment, smokers were informed that they would be participating in a study concerning smoking behaviour and that they were eligible for participation regardless if they wanted to quit or not.

Although the smokers in the experimental group received an intervention, which was focused on changing motivational determinants, all analyses were statistically controlled for the intervention to neutralize any influence on stage transition. Longitudinal analyses could not be conducted for all specific stage transitions (e.g. precontemplation to contemplation, pre-contemplation to preparation, pre-contemplation to action) due to limited sample size. Additionally, respondents making a specific transition were compared to respondents who either remained in the same stage or made the opposite transition. Again, due to sample size, we could not investigate these groups separately.

Some concepts' measures, such as social influence, were limited; we did not assess social pressure and social support and only domestic modelling was measured. However, only a small number of other studies concerning stage transition included social influence in their analyses; they did not find conclusive evidence that these concepts predicted stage transition (De Vries and Mudde 1998).

The I-Change Model's operationalization of preparation differs from that originally proposed by Prochaska et al. (1992), excluding the additional criterion of 
having made a serious quit attempt in the last year. This addition to the operationalization has received much criticism (Sutton 2002), as this contradicts the paradigm itself that no stages are skipped. When including the second criterion, a contemplator without recent quitting experience could never become a preparer and would therefore have to skip preparation and move straight to action. Previous research has also excluded this second criterion (De Vet et al. 2005; Dijkstra et al. 1996).

\section{Recommendations for further research}

Some of the findings are in line with results from previous studies. However, a more unusual finding also surfaced; although self-efficacy is thought to become important when planning to quit in the very near future, our results show that forward transitions from all stages are predicted by a higher self-efficacy rate, including transition from precontemplation and contemplation. These findings are not consistent on both follow-ups, though. However, this suggests that self-efficacy is not a stage-specific predictor, but is an important factor for any smoker moving towards quitting smoking. This is line with Bandura's self-efficacy theory (Bandura et al. 1977) and the assumption that the stages of change are pseudo-stages representing a continuum. However, the results of this study only show a significant influence through the stages for self-efficacy and not for pros, cons or social influence. Although self-efficacy is a continuous predictor of forward stage transition, other cognitive concepts might not be.

Another explanation for the predictive quality of self-efficacy in transitions from pre-contemplation could be the relative heterogeneity of the smokers classified in this stage. All smokers not willing to quit within 6 months are considered to be precontemplators, regardless of what grounds they might have had for their decision (Bandura 1997). However, in previous studies significantly different sub-samples with the pre-contemplation stage have been described (Dijkstra et al. 1997; Kremers et al. 2001). Future research could focus on predicting transitions between these subclassifications as well as to contemplation, preparation and action.

Backward transition from contemplation and preparation appeared not to be predicted by any of the cognitive variables included in the analyses. This was 
unexpected, as previous studies have found significant cognitive predictors of relapse, i.e. regression from action to preparation, contemplation or pre-contemplation (Dijkstra and Borland 2003; Hoving et al. 2006b). We would then have to assume that smokers regress only after a quit attempt. However, from the 108 respondents that did regress to earlier stages within 3 months, only 35 (32\%) indicated to have attempted to quit during that period. After 12 months, 60\% (n=104) of the regressing respondents had attempted to quit smoking after baseline. These figures indicate that not all respondents regressing through the stages have actually relapsed. A change in intention to quit smoking without actually quitting might be predicted by factors other than those measured in this study.

According to Weinstein (1998), the next step in stage transition analyses is examining the effect of matched and mismatched interventions on stage transitions. Although this is a theoretically sound suggestion, the ethical aspect of knowingly providing smokers with possibly unsuitable information might complicate the implementation of deliberately mismatched interventions. However, as it is still not clear whether the stage of change paradigm is a suitable framework on which to base interventions, more research will be necessary to uncover its worth. In particular backward transition is difficult to predict using cognitive predictors, whereas this phenomenon is important. Known non-cognitive predictors of relapse back to smoking might provide a starting point for studies concerning backward transitions from all stages. These include negative affect and nicotine dependence (Kenford et al. 2002; Ockene et al. 2000). Furthermore, it is questionable whether assessing stage of change at baseline and follow-ups is sufficient, as it does not take into account that respondents might have experienced other transitions in between measurements, but is a rather random indication of motivation to change. Although this analyses method has been used in a number of previous studies on stage transition (De Vet et al. 2005; De Vries and Mudde 1998) and it has been acknowledged that persons can move back and forth through the stages (Prochaska and Velicer 1997), future studies should take into account that respondents might not follow the stages of change paradigm in a linear fashion. Therefore, study designs should include more frequent recall of motivation to change by 
respondents and acquiring smoking behaviour information from the period between two measurement points. An active role from the smoker and use of new media, for instance self-report via Internet, might accommodate this more intensive measurement. Not until we can truly map stage transitions over time, do we have an overall view of whether the stage of change paradigm is valuable in the describing behaviour change. 
CHAPTER 8

\section{Predictors of smoking relapse in a sample of Dutch adult smokers; the roles of gender and action plans}

Abstract

The object of this study was to investigate the influence of gender and the additional protective quality of action plans on the occurrence of relapse in smokers after a pharmacy-based minimal intervention strategy. Respondents received a written baseline questionnaire, based on the I-Change Model, and were contacted 3 months after baseline by telephone. A total of 223 respondents were included in relapse analyses. Relapse was predicted by intention, the use of pharmacotherapy and action plans. Predictors differed for men and women. Action plans provide a protective value against smoking relapse. Men and women did not differ in relapse rates, but predictors differed. Developers of relapse prevention activities should consider developing programmes based on gender. These programmes could also benefit from making use of action plans as a method for preventing relapse.

Published as: Hoving EF, Mudde AN, de Vries H (2006). Predictors of smoking relapse in a sample of Dutch adult smokers; the roles of gender and action plans. Addict Behav, 31(7), 1177-1189. 


\section{Introduction}

In the Netherlands around $33 \%$ of the adult population are smokers; $9 \%$ are considered heavy smokers (defined as smoking more then 20 tobacco units a day). More males then females are smokers, respectively $36 \%$ and $28 \%$. The percentage of smokers reaches its peak between 18 and 24 years of age (42\%) (Centraal Bureau voor de Statistiek 2003). Around $88 \%$ of all smokers wish to quit their unhealthy habit (STIVORO for a smokefree future 2001b). As a result, every year about one million smoking cessation attempts are registered in the Netherlands (STIVORO for a smokefree future 1998). Unfortunately, only $7 \%$ of all quit attempts are estimated to be successful (Baillie et al. 1995).

This fallback into smoking after a quit attempt is referred to as smoking relapse. Relapse is a well-documented but unwanted phenomenon that can be seen in studies focusing on the period after the quit attempt, rather than the attempt itself (Krall et al. 2002; Velicer et al. 1990; Willemsen et al. 2002). Most relapse occurs within days or weeks after the initial cessation attempt (Garvey and Bliss 1992). The occurrence of relapse indicates that point prevalence quitting percentages are not the sole outcome to be used when measuring the effect of an intervention, as only participants who remain smoke free truly benefit from their behavioural change. When predictors of relapse within a population are known, intervention developers can use these predictors as a starting point for new interventions incorporating a relapse prevention programme (Green and Kreuter 1991).

Predictors of relapse after a quit attempt have been the subject of many studies. A review including studies measuring short-term relapse (within 6 months) or longer term relapse (12-24 months) describes an older age, male gender, fewer cigarettes smoked per day, a lower addiction level, fewer years smoked and more previous quit attempts as well as a higher self-efficacy and being married as protecting factors against relapse (Ockene et al. 2000). Moreover, Swan et al. (1996) found that 4 weeks after a quit attempt, an increase in both depressed mood and craving predicted relapse. In addition, studies concerning pharmacological aids, such as Nicotine Replacement Therapy (NRT) and bupropion have shown a positive influence on the process of relapse prevention (Hurt et al. 2002; Sanderson Cox et al. 2004). 
However, results and conclusions derived from studies on the influence of gender on relapse are not conclusive. Even though Borelli et al. (2001) found that female gender predicted higher relapse rates within three months after the quit attempt and weight gain predicted relapse for men only, other studies have contradicted these findings. Matheny \& Weatherman (1998) did not find a significant difference in relapse rate for men and women separately and Garvey \& Bliss (1992) did not find any predictors of relapse in relation to gender. Consequently, more research is necessary to uncover a possible relation between gender and relapse.

Previous studies have shown a large number of significant factors, hence the need for a comprehensive model. The concept of relapse has been incorporated in several comprehensive cognitive behavioural models. Marlatt and Gordon's Relapse Prevention Model (Marlatt and Gordon 1985) focuses solely on the description of the determinants of relapse, stating that both overt determinants (high-risk situations, coping skills, outcome expectancies/self-efficacy and the abstinence violation effect) and covert antecedents (lifestyle factors, urges and cravings) are involved in the process of relapse. In the Transtheoretical Model (Prochaska et al. 1997), both behavioural change and relapse are mentioned in connection with the stages of change. An individual is supposed to go through different stages of intention (pre-contemplation, contemplation, preparation) to reach a behavioural change. The action stage is reached when the behaviour is changed, for instance smoking cessation. After the behavioural change has been completed, the individual can either maintain the behavioural change (maintenance) or fall back in the previous behaviour (relapse).

The theoretical framework of this study, the I-Change Model, combines several cognitive behavioural models (De Vries et al. 2003). Its predecessor, the ASE Model (De Vries et al. 1988), has been used as a theoretical base in several previous Dutch studies predicting smoking-related behaviour (Ausems et al. 2002; Bolman and de Vries 1998) and other health behaviours (De Nooijer et al. 2003). The I-Change Model describes three main motivational concepts predicting intention to planned behavioural change (stage of change): attitude, social influence and self-efficacy. These concepts have been recognised by many models and theories as highly relevant when predicting behaviour, although 
appellation can differ. Attitude consists of perceived advantages and disadvantages of a specific behaviour and can be measured by assessing beliefs involving this behaviour and the evaluation of these beliefs. Social influence incorporates three elements: social norm, social pressure and modelling. These elements can be measured by assessing beliefs involving the influence of the social environment on a specific behaviour. Selfefficacy can be measured by assessing perceived capability to perform and maintain a specific behaviour. These three core elements are influenced by predisposing factors, awareness factors (knowledge, risk perception and cues to action) and information factors (the quality, channel and source of information concerning the behaviour change).

In addition to other cognitive models, the I-Change Model acknowledges the influence of the individual's experienced barriers and abilities (implementation plans or action plans and performance skills) on the intention to realise a specific behaviour. Action plans are plans to perform specific reasoned actions that contribute to the transition from intention to behavioural change. These action plans are related to implementation intentions proposed by Gollwitzer (1999) in that they both aim to bridge the intention-behaviour gap. Previous research supports the assumption that implementation intentions positively influence reaching specific health-related goals (Milne et al. 2002; Verplanken and Faes 1999). As the I-Change Model has only recently been developed, only a limited number of studies concerning the influence on smoking behaviour of its additional concept of action plans have been conducted at this time (Hilberink et al. 2005).

The object of this study was to investigate predictors of relapse and the additional value of action plans on its occurrence in adult smokers. In addition, we conducted analyses for males and females separately in order to study the influence of gender on relapse predictors. When predictors of smoking relapse within a population are known, intervention developers can use these predictors as a starting point for new interventions incorporating relapse prevention components (Green and Kreuter 1991). 


\section{Methods}

Procedure

The data used in analyses described in this paper was collected in order to determine the effectiveness of a computer-generated smoking cessation expert system. Pharmacy staff from 65 Dutch community pharmacies were asked to recruit adult (18 years and above) smokers to participate in this study. Smokers were offered a $50 \%$ chance on a computergenerated tailored advice on smoking and smoking cessation. Respondents were randomly assigned to either the experimental group (these smokers received the computer-generated tailored advice) or the control group (these smokers received a thank you letter only). Adults who had smoked in the last seven days were suitable for participating in the study. Smokers who were willing to give their informed consent were then asked to fill in the questionnaire. In total, 915 smokers from both the experimental and control group returned their questionnaire and informed consent. Three months after baseline, all respondents were contacted by telephone for follow-up.

Respondents were included in the analyses when they had completed the baseline questionnaire, their smoking status was available at follow-up and when they had made a quit attempt between baseline and follow-up after 3 months. No data could be obtained from participants that refused to participate in the study at baseline.

\section{Baseline questionnaire}

The written baseline questionnaire, consisting of 54 questions, has been tested experimentally (Dar and Frenk 2002; Dijkstra et al. 1996; Dijkstra et al. 1998b) and is currently the basis of a computer-generated tailored smoking cessation programme distributed nationwide by the Dutch Foundation on Smoking and Health (STIVORO for a smokefree future). By means of this questionnaire we collected demographic data (gender, age, addiction level, the occurrence of cardiovascular and respiratory diseases), number of previous quit attempts, intention to quit smoking and action plans concerning smoking cessation, as well as motivational determinants (attitude, self-efficacy and social influence). 
Addiction level was measured with 3 items, using the abbreviated Fagerström scale (Heatherton et al. 1991)): assessing the type of tobacco product smoked, the amount of tobacco smoked (in number of cigarettes) and time after tobacco is smoked when getting out of bed (within 5 minutes, between 6 and 30 minutes, 31-60 minutes and after 60 minutes). Respondents were scored on a 7-point scale on basis of their answers $(0=$ not addicted, 6=highly addicted). This scale was used in analyses.

The occurrence of cardiovascular and respiratory diseases was measured by one dichotomous item each (I suffer from a cardiovascular disease, I suffer from a respiratory disease; $0=$ no, $1=y e s)$.

Previous quit attempts were assessed by 2 items, asking respondents if they had ever seriously tried to quit smoking and how often they had attempted to quit.

Questions concerning the motivational determinants attitude and self-efficacy were formulated as statements; participants were asked to indicate on a 5-point scale to what extent they agreed with the statement.

Attitude was assessed using 20 items ( $0=$ disagree/do not know, (-)1= somewhat agree, (-) 2=agree, (-) 3=fully agree): positive outcome expectations of quitting or pros (improved own health and that of others, financial benefit), negative cognitive consequences or cognitive cons of smoking cessation (weight gain, boredom, withdrawal symptoms) and emotional or affective cons of smoking (dissatisfaction, shame and regret). Higher scores on positive outcome expectations implied seeing more pros, whereas higher scores on both cognitive and emotional cons implied seeing less cognitive or affective cons.

Self-efficacy was measured by 16 items (-2=disagree, 2=agree): respondents were asked to indicate if they would be able to refrain from smoking in emotional, social and habitual situations and when confronted with negative outcomes of the cessation attempt.

Two items assessed social influence measuring smoking status of the partner (yes, no, not applicable) and residential children (all, the majority, half, a minority, none, not applicable). The items were each transformed into 3 category-scales for inclusion in analyses: (-1 non-smoking, 0 not applicable, 1 smoking). 
Intention to quit smoking was assessed with one item by asking respondents to state within what period they intended to quit smoking on a 7-point scale (within 1 month, within 6 months, within 1 year, within 5 years, at some point but not within 5 years, never quit but smoke less or use tobacco products with lower nicotine and tar levels and never quit or smoke less or use tobacco products with lower nicotine and tar levels). For analysing purposes, the 7-point scale was reduced to three categories of the stages of change: pre-contemplator (not to quit within 6 months), contemplator (to quit within 6 months) and preparer (to quit within 1 month).

Action plans were assessed by 5 items asking respondents to indicate whether they would use a specific action plan if they attempted to quit smoking (quit immediately, remove all smoking materials from the home, inform people around me, seeking cessation aids and prospecting a reward for myself).

\section{Follow-up}

At follow-up, participants were asked about their current smoking status, smoking activity in the last 3 months and whether they had used pharmacotherapy (NRT or bupropion) if they had attempted to quit after baseline. Educational level and year of birth were also assessed.

Analysis

Data from baseline and follow-up were analysed using SPSS Version 11.0. Attrition analyses were conducted in order to rule out potential response bias. For analyses, differences between relapsers and maintainers were assessed. Respondents who had quit smoking between baseline and follow-up for at least 24 hours on end and smoked in the 7 days before follow-up were identified as relapsers; respondents who had quit smoking in the same period but had not smoked in the last 7 days were identified as maintainers.

Firstly, as the I-Change Model assumes specific clusters of items within the motivational concepts attitude and self-efficacy, a factor analysis using oblique rotations was run for these concepts. The items within the factors were combined into a mean score for each factor. In order to allow for analyses concerning interaction effects, z- 
scores were calculated for these factors as well as the items concerning social influence. A set of z-scores was calculated for the total sample as well as for men and women subsamples separately. All subsequent analyses were controlled for condition (experimental or control group).

Secondly, attrition analysis by means of univariate logistic regression was conducted.

Thirdly, univariate logistic regression was used for analysing continuous and dichotomous variables to determine differences between relapsers and maintainers.

Fourthly, backward stepwise logistic regression analysis was performed. Items and factors were included in the regression in 4 blocks: 1) demographic variables), 2) motivational determinants, 3) intention to quit smoking and action plans, 4) use of pharmacotherapy. To neutralise any effect the intervention might have had on transitions through the stages, the concept of condition remained in the analyses as a covariate, regardless of its significance level. Variables were excluded from the equation based on change in the model's likelihood ratio. The exclusion criterion was set at 0.20 , as recommended by Hosmer and Lemeshow (1989), in order to compensate for stepwise analysis. Interaction effects for gender were calculated by means of logistic regression analysis to justify separate analyses for men and women. Partial correlations between variables included in the analyses were also calculated.

\section{Results}

Sample

A total of 223 respondents met the criteria for inclusion in the analyses. Baseline characteristics are shown in table 8.1.

Attrition analyses revealed that respondents included in the analyses were significantly older $(\mathrm{OR}=1.02, \mathrm{p}<.01)$ and had a stronger intention of quitting smoking $(\mathrm{OR}=0.84, \mathrm{p}<.05)$ than respondents lost to follow-up. No significant differences in level of education, addiction level, number of previous cessation attempts or the occurrence of cardiovascular and pulmonary diseases were found. Interaction analysis revealed no significant effects in relation to gender; separate attrition analyses for men and women 
were therefore not conducted. Collinearity diagnostics revealed no existing multicollinearity or singularity (Tabachnick and Fidell 2001).

Table 8.1.

Baseline characteristics of respondents included in analyses $(N=223)$.

\begin{tabular}{|c|c|c|}
\hline \multicolumn{2}{|c|}{ Female gender } & $58 \%$ \\
\hline \multicolumn{2}{|c|}{ Age (mean) } & 46 \\
\hline \multicolumn{3}{|c|}{ Education } \\
\hline- & Primary school/basic vocational school & $25 \%$ \\
\hline- & Secondary vocational school/high school degree & $44 \%$ \\
\hline- & Higher vocational school/college degree/university degree & $31 \%$ \\
\hline \multicolumn{3}{|c|}{ Health } \\
\hline- & Occurrence of cardiovascular diseases & $12 \%$ \\
\hline- & Occurrence of chronic pulmonary diseases & $23 \%$ \\
\hline \multicolumn{3}{|c|}{ Home situation } \\
\hline- & Smoking partner & $40 \%$ \\
\hline- & Smoking residential children & $9 \%$ \\
\hline \multicolumn{3}{|c|}{ Tobacco use } \\
\hline- & Cigarettes & $48 \%$ \\
\hline- & Cigarette tobacco & $29 \%$ \\
\hline- & Cigars & $2 \%$ \\
\hline- & Pipe tobacco & - \\
\hline- & Using $>1$ type of tobacco & $20 \%$ \\
\hline \multicolumn{3}{|c|}{ Stage of Change } \\
\hline- & Pre-contemplator & $21 \%$ \\
\hline- & Contemplator & $19 \%$ \\
\hline- & Preparer & $60 \%$ \\
\hline \multicolumn{2}{|c|}{ Daily consumption in cigarettes (mean) } & 21 \\
\hline \multicolumn{2}{|c|}{ Quit attempt during study } & $100 \%$ \\
\hline \multicolumn{2}{|c|}{ Relapse during study } & $73 \%$ \\
\hline \multicolumn{2}{|c|}{ Use of NRT with quit attempt during study } & $14 \%$ \\
\hline \multicolumn{2}{|c|}{ Use of bupropion with quit attempt during study } & $14 \%$ \\
\hline
\end{tabular}

\section{Coherence within motivational concepts}

The factor analysis revealed three attitude factors: the advantages of smoking cessation $(\alpha=.5035)$, the cognitive disadvantages of smoking cessation $(\alpha=.8377)$ and affective disadvantages of smoking $(\alpha=.6108)$. Social influence items were not combined into one 
construct $(\alpha=.2706)$. Four self-efficacy factors were found: self-efficacy to refrain from smoking in social situations (social self-efficacy, $\alpha=.8610$ ), self-efficacy to refrain from smoking when in an emotional state (emotional self-efficacy, $\alpha=.8697$ ), self-efficacy to refrain from smoking when confronted with own habits (habitual self-efficacy, $\alpha=.8680$ ) and self-efficacy when coping with negative outcomes of smoking cessation (negative outcome self-efficacy, $\alpha=$.6565).

Relapse

Table 8.2 consists of results of the univariate logistic regression analyses concerning smoking relapse.

Relapsers had experienced more previous cessation attempts than maintainers and experienced more affective cons concerning the smoking habit. Relapsers were more likely to prefer the action plan to remove all smoking materials from the home and use NRT during the preceding quit attempt.

Interaction effects were found between gender and addiction level, habitual self-efficacy and negative outcome self-efficacy. Consequently, analyses for men and women separately were conducted additionally. Table 8.3 summarises the predictors of relapse as a result of logistic regression analyses for the complete sample and for men and women separately.

Respondents were more likely to relapse if they had quit more often in the past and if they were more motivated to quit at baseline. Using bupropion during the quit attempt predicted against relapse. The preference for the action plan to remove all smoking materials from the home was a borderline significant predictor for relapse. The proposed model explained $20 \%$ of the variance.

Unlike in univariate analyses, the use of NRT and affective cons concerning smoking were not significant predictors of relapse. Univariate logistic regression analyses were conducted in order to determine whether a third variable might mediate the relation between NRT or affective cons concerning smoking and relapse. NRT use correlated significantly with addiction level only $(\mathrm{r}=.14, \mathrm{p}<.05)$. 
However, univariate logistic regression analyses controlled for condition and addiction level or any other single variable in the final model did not neutralise the influence of NRT on relapse rates. Bivariate correlations showed significant correlations between affective cons of smoking with education $(\mathrm{r}=.15, \mathrm{p}<.05)$ and number of previous cessation attempts $(\mathrm{r}=-.18, \mathrm{p}<.01)$. However, univariate logistic regression analyses controlled for condition and education or number of previous cessation attempts or addiction, the other single variable in the model at that time, did not mediate the effect of affective cons on relapse rates.

Table 8.2.

Odds ratios and means (adjusted for condition) concerning smoking relapse ( $N=223$ ).

\begin{tabular}{|c|c|c|c|c|c|}
\hline & \multirow{2}{*}{$\begin{array}{c}\text { Mean } \\
\text { Relapser }\end{array}$} & \multirow{2}{*}{$\begin{array}{c}\text { Mean } \\
\text { Maintainer }\end{array}$} & \multirow[t]{2}{*}{ OR } & \multicolumn{2}{|c|}{$95 \% \mathrm{CI}$} \\
\hline & & & & Lower & Upper \\
\hline Gender & 1.59 & 1.56 & 1.12 & 0.62 & 2.03 \\
\hline Age & 45.97 & 46.09 & 1.00 & 0.98 & 1.02 \\
\hline Education & 3.95 & 4.28 & 0.89 & 0.74 & 1.06 \\
\hline Addiction level & 3.25 & 2.79 & 1.17 & 0.98 & 1.39 \\
\hline Previous cessation attempts & 3.47 & 2.24 & $1.16^{*}$ & 1.01 & 1.34 \\
\hline Cardiovascular diseases & 0.12 & 0.10 & 1.30 & 0.50 & 3.41 \\
\hline Chronic pulmonary diseases & 0.23 & 0.23 & 0.99 & 0.49 & 2.00 \\
\hline Stage of Change & 2.49 & 2.15 & 1.64 & 1.15 & 2.34 \\
\hline Pros of cessation & 0.06 & -0.16 & 1.25 & 0.93 & 1.68 \\
\hline Cognitive cons of cessation & -0.03 & 0.09 & 0.88 & 0.65 & 1.19 \\
\hline Affective cons of smoking & -0.10 & 0.26 & $0.69^{*}$ & 0.50 & 0.94 \\
\hline Smoking partner & 0.04 & -0.11 & 1.18 & 0.87 & 1.59 \\
\hline Smoking children & -0.04 & 0.10 & 0.87 & 0.64 & 1.17 \\
\hline Social SE & -0.02 & 0.06 & 0.92 & 0.68 & 1.24 \\
\hline Habitual SE & -0.05 & 0.12 & 0.84 & 0.62 & 1.14 \\
\hline Emotional SE & -0.05 & 0.14 & 0.83 & 0.62 & 1.11 \\
\hline Negative outcome SE & -0.08 & 0.20 & 0.75 & 0.55 & 1.02 \\
\hline AP: quit immediately & 0.80 & 0.84 & 0.80 & 0.37 & 1.74 \\
\hline AP: remove smoking materials & 0.69 & 0.51 & $2.18^{*}$ & 1.19 & 3.98 \\
\hline AP: inform people & 0.61 & 0.51 & 1.53 & 0.84 & 2.77 \\
\hline AP: seek aids & 0.65 & 0.59 & 1.29 & 0.70 & 2.35 \\
\hline AP: prospect reward & 0.32 & 0.31 & 1.05 & 0.56 & 1.98 \\
\hline Use of NRT & 0.17 & 0.05 & $4.05^{*}$ & 1.18 & 13.85 \\
\hline Use of bupropion & 0.12 & 0.20 & 0.55 & 0.25 & 1.21 \\
\hline
\end{tabular}

\footnotetext{
${ }^{*} \mathrm{p}<.05 .{ }^{* *} \mathrm{p}<.01$.
} 
Table 8.3.

Predictors of relapse, adjusted for condition, for total sample as well as men and women separately ( N=223).

\begin{tabular}{|c|c|c|c|c|c|c|c|c|c|}
\hline & \multicolumn{3}{|c|}{ Total sample $(n=223)$} & \multicolumn{3}{|c|}{ Males $(n=94)$} & \multicolumn{3}{|c|}{ Females $(n=129)$} \\
\hline & \multirow[t]{2}{*}{ OR } & \multicolumn{2}{|c|}{$95 \%$ CI } & \multirow[t]{2}{*}{ OR } & \multicolumn{2}{|c|}{$95 \% \mathrm{CI}$} & \multirow[t]{2}{*}{ OR } & \multicolumn{2}{|c|}{$95 \% \mathrm{CI}$} \\
\hline & & Lower & Upper & & Lower & Upper & & Lower & Upper \\
\hline Education & 0.86 & 0.70 & 1.07 & 0.74 & 0.52 & 1.04 & -1 & -1 & -1 \\
\hline $\begin{array}{l}\text { Addiction } \\
\text { level }\end{array}$ & 1.14 & 0.94 & 1.39 & ${ }^{1}$ & -1 & -1 & $1.36^{*}$ & 1.04 & 1.79 \\
\hline $\begin{array}{l}\text { Number of } \\
\text { previous } \\
\text { cessation } \\
\text { attempts }\end{array}$ & $1.16^{*}$ & 1.01 & 1.34 & 1.18 & 0.96 & 1.44 & 1.21 & 0.97 & 1.50 \\
\hline $\begin{array}{l}\text { Affective } \\
\text { cons of } \\
\text { smoking }\end{array}$ & 0.83 & 0.57 & 1.20 & 0.77 & 0.41 & 1.45 & -1 & -1 & $-^{1}$ \\
\hline $\begin{array}{l}\text { Social self- } \\
\text { efficacy }\end{array}$ & $-^{1}$ & -1 & $-^{1}$ & 1.26 & 0.58 & 2.73 & -1 & -1 & ${ }^{1}$ \\
\hline $\begin{array}{l}\text { Habitual self- } \\
\text { efficacy }\end{array}$ & $-^{1}$ & -1 & -1 & 0.67 & 0.32 & 1.38 & ${ }^{1}$ & -1 & $-^{1}$ \\
\hline $\begin{array}{l}\text { Negative } \\
\text { outcome self- } \\
\text { efficacy }\end{array}$ & ${ }^{1}$ & ${ }^{1}$ & ${ }^{1}$ & -1 & -1 & ${ }_{-1}^{1}$ & $0.53^{* *}$ & 0.32 & 0.87 \\
\hline Smoking & & & & & & & & & \\
\hline Partner & $-^{1}$ & -1 & $-^{1}$ & $1.89^{*}$ & 1.02 & 3.50 & -1 & $-^{1}$ & $-^{1}$ \\
\hline $\begin{array}{l}\text { Stage of } \\
\text { Change }\end{array}$ & $1.56^{*}$ & 1.03 & 2.36 & $2.60^{*}$ & 1.19 & 5.67 & 1.74 & 0.97 & 3.10 \\
\hline $\begin{array}{l}\text { AP: quit } \\
\text { immediately }\end{array}$ & $-^{1}$ & $-^{1}$ & $-^{1}$ & 0.20 & 0.04 & 1.02 & -1 & $-^{1}$ & $-^{1}$ \\
\hline $\begin{array}{l}\text { AP: remove } \\
\text { all smoking } \\
\text { materials } \\
\text { from home }\end{array}$ & 1.94 & 1.00 & 3.79 & 2.28 & 0.76 & 6.87 & 2.38 & 0.93 & 6.14 \\
\hline $\begin{array}{l}\text { AP: inform } \\
\text { people }\end{array}$ & & & & & & & & & \\
\hline around me & $-^{1}$ & $-^{1}$ & $-^{1}$ & ${ }^{1}$ & -1 & -1 & 2.41 & 0.94 & 6.23 \\
\hline $\begin{array}{l}\text { AP: seek } \\
\text { cessation aids }\end{array}$ & ${ }^{1}$ & ${ }_{-}^{1}$ & ${ }^{1}$ & ${ }^{1}$ & ${ }^{1}$ & ${ }^{1}$ & 0.44 & 0.16 & 1.22 \\
\hline Use of NRT & 2.89 & 0.80 & 10.44 & -1 & ${ }^{1}$ & ${ }^{1}$ & 3.23 & 0.53 & 19.62 \\
\hline $\begin{array}{l}\text { Use of } \\
\text { bupropion }\end{array}$ & $0.38^{*}$ & 0.15 & 0.92 & 0.20 & 0.03 & 1.42 & -1 & $-^{1}$ & $-^{1}$ \\
\hline $\begin{array}{l}\text { Nagelkerke's } \\
\text { R square }\end{array}$ & & .20 & & & .34 & & & .28 & \\
\hline
\end{tabular}

${ }^{*} \mathrm{p}<.05 .{ }^{* *} \mathrm{p}<.01$.

${ }^{1}$ Concept was not included in the final model. 


\section{Gender differences}

For men only, relapse could be predicted by a higher intention to quit smoking at baseline. A smoking partner also increased the risk of a relapse. The preference for the action plan to quit immediately was a borderline significant predictor against relapse. The proposed model explained $34 \%$ of the variance.

Women were more likely to relapse if they had a higher addiction level and a higher intention to quit smoking at baseline. A higher intention to quit smoking was a borderline significant predictor of relapse. The proposed model explained $28 \%$ of the variance.

\section{Conclusions and discussion}

In this study, predictors of smoking relapse were investigated within a sample of Dutch adults, with emphasis on gender and action plans.

\section{General conclusions}

A higher intention to quit smoking at baseline was a significant predictor of relapse. This was to be expected, as preparers were over-represented in the sample used for relapse analyses. After all, smokers in the preparation stage are most likely to quit smoking, which also means that they have more chance of relapsing.

Smokers who had experienced a higher number of cessation attempts in the past were also more likely to relapse. This seems to somewhat contradict previous research, as experience with smoking cessation is considered to be a predictor of maintenance (Ockene et al. 2000). However, more previous quit attempts can also indicate the smoker being less serious about these attempts. The length of these previous attempts is also of importance; if the smoker managed to maintain a non-smoking status for a longer period before, the chance of managing this again will increase (Ockene et al. 2000).

The use of NRT was not a significant predictor of relapse, when controlled for other variables. This confirms outcomes of a review by Silagy et al. (2004), reporting that the effectiveness of NRT appears largely to be independent of the intensity of additional 
support provided to the smoker. Additionally, the use of bupropion during the attempt decreased the chance of relapse, complementing results from previous effectiveness studies (Hughes et al. 2004b).

\section{The influence of gender on relapse}

No differences in relapse rates between men and women were found. This confirms results from a study conducted by Matheny \& Weatherman (1998). Relapse in men was predicted by occurrence of a smoking partner and a higher intention to quit smoking, whereas low self-efficacy concerning negative outcomes (craving, regret and weight gain) and a higher addiction level predicted relapse in women. A previous study (Borrelli et al. 2001) suggested that men who gained weight after quitting smoking were more likely to relapse. This could indicate that men underestimate the impact of weight gain on their quitting behaviour. Although it has been suggested in previous studies that NRT is less suitable for women than for men as a cessation method (Monso et al. 2001; Wetter et al. 1999), we did not find the use of NRT to be a predictor of relapse related to gender.

The influence of action plans on relapse

The results show that action plans have a significant positive effect on smoking maintenance. The preference for the action plan to remove all smoking materials from the home was a borderline significant predictor for relapse for both total sample and women only. Although these plans appear to be helpful in quitting smoking, the methods alone apparently did not protect against relapses. A combination of several action plans (for instance informing people about the smoking attempt while also removing all smoking materials from the home) might increase the likeliness of a successful quitting attempt. It is also possible that a successful action plan alone is not enough to prevent relapse. 


\section{Limitations of this study}

This study is subject to some limitations. First, results and conclusions of this study are based on self-reported smoking behaviour. However, results of previous scientific studies (Glasgow et al. 1993; Patrick et al. 1994; Velicer et al. 1992) suggest that the difference between self-reported abstinence rates and those verified with biochemical validation is negligible. Moreover, as there were no negative consequences of relapsing during this study, there was no direct reason for the respondents to give wrong information about their smoking status.

Second, as the sample was self-selected, this could have introduced selection bias in the study. The relative large number of respondents in the preparation stage of change confirms this. However, this does not diminish the results of this study. The study did not focus on relapse rates after an intervention, but looked merely at the predictors of relapse. Therefore, selection bias concerning stage of change did not influence results.

Thirdly, instead of measuring intention to remain smoke-free directly, we used an indirect measure: the intention to quit smoking. However, this measure also plays a part in the relapse process; it is to be expected that smokers indicating that they intend to quit smoking also have the intention to remain smoke free. Therefore, we believe the use of intention to quit as a measurement relating to the processes of relapse to be a valid choice.

Fourthly, the measurement of action plans was limited. Only a small number of possible action plans was offered and respondents did not have the opportunity to formulate action plans themselves. Preceding research showed that the benefit of making action plans increases when individuals formulate their own plans (Gollwitzer 1999). More thorough measurement of action plans will give a better indication of which role these plans play in cessation and relapse behaviour.

Fifthly, we have not corrected our results for type 1 error. However, to compare our results with previous work that did not report to have performed this correction, we felt that the same level of strictness would be appropriate. 
Finally, due to the fact that the questionnaire had to be brief, we could not assess all factors elaborately. Hence, we did not include, for instance, affective factors such as level of depression that have been found to be related to relapse (Ockene et al. 2000).

\section{Recommendations}

Quitters in our study seemed to underestimate the difficulty of durable cessation as almost $75 \%$ respondents experienced a relapse within the three-month period of this study. This shows the importance of relapse prevention interventions. The goal of these interventions should be to improve maintenance rates by providing quitters-to-be with enough coping devices to deal with the danger of relapse and prevent its occurrence. By using more than one strategy, chances improve of addressing the right issues and facilitating specific needs. The results of this study, in addition to previous studies findings, can be used as a base for relevant topics to be addressed by the prevention programme. An important factor to take into account is gender; although in the present study relapse rates did not differ between men and women, the predictors of relapse were gender-related, indicating different programme contents for men and women. However, it must be kept in mind that this study was conducted within a Western society. As previous studies show that ethnicity does not play a major role in reasons for smoking and smoking behaviour (Gallant and Dorn 2001; Sanchez-Johnsen et al.) within Western communities, this could also be the case for smoking relapse. Nonetheless, in other cultures smoking may serve different roles, possibly resulting in other determinants of relapse. Moreover, the acceptance of smoking for women may differ over cultures (Morrow and Barraclough 2003). Further research concerning relapse predictors among non-Western populations is therefore advisable.

To prevent relapse by using more than one strategy, chances improve of addressing the right issues and facilitating specific needs. Our study suggests that a programme should include factors such as previous quitting experience and intention to quit smoking. This previous quitting experience could either consist of serious or halfhearted quit attempts. To have a better chance of succeeding in quitting smoking, smokers should be informed about the possible difficulties to be encountered while 
attempting to quit, thus being able to realistically revalue their intention to quit smoking. The use of pharmacotherapy as an additional cessation aid should also be discussed.

Designers of relapse prevention programmes should consider developing programmes for men and women separately. Special focus should be directed towards women with a high addiction score and a low negative outcome self-efficacy score. Additionally, action plans seem to play a part in the prediction of relapse for men and women and should be given attention accordingly.

Future research on relapse using a more elaborate model is recommended to be better able to study the impact of more factors that have been identified in the literature as potential predictors. 

CHAPTER 9

\section{General Discussion}

Abstract

In this chapter, results from the studies described in previous chapters are combined, discussed and put into context of previously conducted research. Furthermore, theoretical and methodological issues will be discussed, as well as implications for future research and practical implications. 
The present thesis provides an overview of studies conducted to test the effectiveness of a computer-generated tailored expert system and its utilization in Dutch community pharmacy and general practice. Glasgow et al. (2003) presents intervention impact as effect $\mathrm{x}$ reach $\mathrm{x}$ implementation; each of these concepts are discussed within chapters 2 to 8 . In summary, this chapter will discuss six main research questions that were brought forward in chapter 1:

1. What determines dissemination (adoption and implementation) of the expert system in Dutch general practice

2. How do community pharmacy assistants evaluate using the expert system after a triability period of three months and are they motivated to further implement the expert system?

3. What is the effectiveness of the expert system within community pharmacy and general practice setting?

4. How do recruitment method and setting affect the samples acquired and how do these samples relate to the Dutch adult smoking population?

5. Can smokers in different stages of change be differentiated from each other by cognitive factors and do different sets of cognitive factors predict different stage transitions?

6. What are smokers' cognitive predictors of relapse after a quit attempt? 
These questions were identified through application of the I-Plan Model (De Vries 1998) to the risk behaviour of tobacco smoking, as presented in chapter 1. A summary of the findings pertaining to these questions will be presented, methodological issues regarding the performed studies will be described and recommendations following our findings will be presented.

\section{1: Adoption and implementation in general practice setting}

In chapters 2 and 4, intentions to initially adopt and further implement the expert system were measured among general practice staff. Based on assumptions from the I-Change Model, as well as from previous research (Segaar et al. 2006), we expected adopters and implementers to report significantly more pros of using the expert system, have a higher self-efficacy towards using the expert system and perceive social influences towards performing smoking cessation activities in general practice. Analyses conducted in chapter 2 confirmed these assumptions regarding adoption. In addition, GPs mostly regarded smoking cessation activities as a task for the practice assistant. This could indicate that general practitioners were positive towards the expert system due to delegation of the expert system to their practice assistants. This is reflected in the results from chapter 4; respondents willing to further implement the expert system were more likely to be GPs.

In chapter 4, the assumptions were also confirmed with respect to implementation willingness, except for the perceived benefits of using the expert system which did not determine further implementation of the expert system. In contrast, a study previously conducted showed that implementation of a smoking cessation intervention was only determined by perceiving more advantages, whereas the concepts of social influence and self-efficacy did not reach significance (Bolman et al. 2002). The Diffusion of Innovation theory also specifies the relative advantage of the intervention as a determinant of the adoption rate (Rogers 2002). Univariate testing within our sample showed that implementers did perceive more pros of using the expert system than nonimplementers, but its predictive quality was neutralized within multivariate logistic regression analyses. Other concepts included in the final multivariate model, such as the 
level of self-efficacy, might have neutralized the predictive quality of perceived advantages. This could partly account for the differences in determinants found. Furthermore, our analyses included both assistants and GPs, whereas the study by Bolman et al. included nurses working on cardiac wards. Analyses only including general practice assistants also showed that perceived pros predicted intending to implement the expert system and not self-efficacy or social influence, although these results should be interpreted with extreme caution due to small sample size $(n=48)$.

\section{Recommendations}

Our findings suggest that smoking cessation interventions in general practice should be accompanied by a recruitment approach addressing general practice staff and their social environment as a whole (including direct colleagues) and improving self-efficacy towards applying the intervention. This approach could include employing current adopting or implementing colleagues in the recruitment process and providing extensive feedback after role-playing or acquiring skills in daily practice. Supporting this strategy is the finding that implementers were more likely to have actively offered the expert system to their patients.

Although a more positive attitude towards the intervention did not determine implementation willingness in our study, its influence should be assessed more thoroughly. Subsequent studies could focus on identification of relevant additional items to improve the measurement's validity and apply longitudinal study designs to assess intention willingness and subsequent dissemination behaviour.

Future research should also specifically focus on mapping determinants of prevention intervention dissemination and increasing adoption and implementation willingness among practice assistants, as tasks concerning smoking cessation interventions are likely to be delegated to these health professionals. Furthermore, additional groups working within general practice, such as practice nurses, should be included in dissemination studies. Practice nurses can increase the quality of care and

relieve GPs by providing care and counselling to specific chronic patient groups, such as diabetes and asthma patients (Lamkaddem et al. 2004), including lifestyle advice. 


\section{2: Feasibility and continuation in community pharmacy setting}

Chapter 5 described pharmacy assistants' experiences using the expert system in their pharmacy and their intention to further implement the expert system into their daily practice. We were interested in pharmacy assistants' attitudes towards the expert system, perceived influences from colleagues, expressed confidence to approach smokers and offer the expert system and their intention to continue using the expert system in their pharmacy.

Overall, pharmacy assistants expressed a positive attitude towards smoking cessation activities, but were unsure whether community pharmacies were a suitable channel. This is contrary to international studies, where pharmacy personnel indicated patient counselling to be an important subject in pharmacy practice (Berger et al. 2005; Williams et al. 2000). The community pharmacy setting has received limited attention as a channel for health promotion activities in the Netherlands; this might have influenced the pharmacy assistants' attitude towards smoking cessation activities.

Furthermore, approaching smokers without smoking-related complaints or not actively showing interest in quitting was regarded as a barrier; assistants felt they lacked skills on how to approach these smokers. A previous study (Berger et al. 2005) also showed that counselling was most likely to be given on request by the patient or when prompted by a symptom or a request for a specific medicine. It may be that approaching all clients - as was the intended in our study - may not have been the best strategy and may have caused assistants to feel uncomfortable with this new active role. Furthermore, as pharmacy assistants have not often been approached to deliver health promotion interventions, they might have lacked specific skills necessary to deal with the tasks expected of them within this project.

Also, pharmacy assistants indicated that some smokers were approached more than once during recruitment; this caused feelings of irritation among approached smokers which might have resulted in negative feelings regarding active recruitment among the pharmacy assistants. However, it is possible that the actual number of clients approached more than once was limited, but that the experience had a large impact on the assistants' subsequent behaviour and the evaluation of their role. Avoidance of 
recruitment will limit its intensity and effectiveness, possibly partly explaining the insignificant difference in the number of recruited smokers between active and passive recruitment in community pharmacy setting (Hoving et al. 2007).

In sum, pharmacy assistants indicated becoming discouraged by negative responses from clients and perceived the active recruitment strategy as too intensive. Although active recruitment methods have shown to be able to reach more individuals and obtain larger samples than passive recruitment strategies (Friebel et al. 2004; McDonald 1999), active recruitment may have been too intensive for pharmacy assistants at this time. According to the I-Change Model, negative experiences with performed behaviour can lower beliefs regarding the advantages and decrease feasibility of using the expert system, which then can lead to a lower intention to actively approach new clients (De Vries et al. 2003). A less invasive recruitment strategy might therefore be more suitable for use in community pharmacy setting in the Netherlands.

\section{Recommendations}

Pharmacy assistants were relatively negative towards actively offering the expert system, as they reported a lack in skills to approach all smokers. Additional training might be helpful to increase the assistant's skills and self-efficacy to engage in conversation regarding a client's smoking habit and provide information on their options to quit. Previous studies have shown that knowledge and self-efficacy, as well as a more positive attitude towards smoking cessation activities in community pharmacy, can be increased by providing skills training to pharmacy staff (Sinclair et al. 2004; Zillich et al. 2004). As pharmacy assistants also indicated not being able to consistently actively approach all clients due to time constraints, training and counselling could possibly be limited to a small number of practice assistants in each pharmacy, which then can also act as a smoking cessation contact when implementing other innovative interventions, thereby further structuring the smoking cessation activities in community pharmacy setting.

Furthermore, pharmacy assistants may benefit from external guidance to adequately offer the expert system in their daily practice, as was found in a previous 
Dutch study regarding application of patient education in community pharmacy (Pronk 2001). This could take shape as a question hotline, ongoing possibilities for training or recruitment materials such as posters and leaflets. However, pharmacies included in our study were offered some external guidance (posters, FAQ booklet, frequent telephone calls to assess recruitment), but assistants still found active recruitment to be unfeasible for the entire study period. Training should therefore be the focal point of improving willingness to play an active role in health promotion.

In addition, future smoking cessation interventions in community pharmacy setting might apply measures to avoid re-approaching of clients. Firstly, the intervention period could be shortened in order to maintain motivation in the pharmacy personnel. A UK-based study showed that three pharmacies actively recruiting (in a similar fashion to our study) resulted in 75 smokers within one month (Lloyd-Williams 2003), showing that a fairly large number of smokers can be recruited within a limited timeframe. Possibly, actively offering of the expert system could be limited to specific short periods to start implementation of the expert system in Dutch community pharmacy. Secondly, a link to the pharmacy's administration could provide details as to whether a client has yet been approached, possibly with their individual response to the recruitment effort. However, the effectiveness of this link depends on whether patients' smoking status is updated regularly. Thirdly, a collection of different recruitment strategies could be applied, such as invitations by letter or telephone, increasing flexibility to initiate recruitment and limiting time taken away from pharmacy core tasks. Previous studies have shown that these strategies can be effective in recruiting smokers (Holtrop et al. 2005; McClure et al. 2006)

\section{Comparing general practice and community pharmacy setting}

Overall, responses from general practice regarding the expert system were more positive than those from community pharmacy setting. GP staff reported a positive attitude and self-efficacy towards using the expert system in the future, whereas pharmacy assistants indicated feelings of uncertainty and insecurity with the use of the expert system in their pharmacy. However, general practices applied a passive recruitment method, whereas 
pharmacy assistants actively recruited smoking customers. The significantly more active role of practice assistants might have led to a more negative view on the use of the expert system. This assumption is strengthened by the finding that if pharmacy assistants indicated that if the use of the expert system was continued within their practice, they would offer it passively or actively within a very limited time period.

As both settings differed in recruitment strategy, it is not possible to indicate which setting is most effective on the basis of our studies. Unfortunately, we did not assess responses from the 15 pharmacies using the passive recruitment approach, as was used in general practices. By comparing these two settings, we would have been able to assess differences in descriptive and cognitive factors, as well as intention and implementation rate in direct relation to recruitment setting.

\section{Recommendations}

Future studies should involve the comparisons as suggested above. Furthermore, additional studies could investigate how and if specific delivery methods (e.g. via telephone, face-to-face, personal invitation letter) influence active recruitment (cost) effectiveness within health care settings.

\section{3: Effect of the expert system}

In chapter 3, the effect of the expert system on smoking outcome measures was tested for general practice and community pharmacy setting separately. On the basis of a previous efficacy study regarding the expert system (Dijkstra et al. 1998d), we hypothesized that the expert system would result in $14 \%$ more quit attempts among preparers.

Our findings from both the general practice and community pharmacy sample and all follow-up measurements show a 7-11\% difference in quit attempts between preparers in the experimental and control group, therefore not reaching the hypothesized $14 \%$ difference. However, after 12 months did the expert system have a significant effect on quitting activity within the preparer stage in the pharmacy-recruited sample, but not on abstinence measures and not for contemplators. This is in contrast with results found from a previous efficacy study of the expert system, showing 
significant long-term (14 months after baseline) effects of the expert system on quit attempt and continued abstinence in a sample including motivated and unmotivated smokers (Dijkstra et al. 1998b).

How can we explain the failure to replicate findings from previous studies regarding the expert system? Firstly, these studies have been efficacy trials, focusing on the effect of the expert system under optimal conditions. Respondents were recruited through newspaper advertisements and were invited to pro-actively approach the research team directly. Furthermore, smokers were eligible for money prizes when completing all study questionnaires (Dijkstra et al. 1998d). As a result, respondents were likely more motivated to participate in the study than respondents within our sample. Additionally, their participation did not depend on how much time and effort intermediates spent recruiting. Efficacy trials are not designed to systematically mimic real-life circumstances; main focus is the effect of the intervention among respondents without practical barriers. Nevertheless, the choice for large-scale implementation of interventions often relies on results from efficacy trials (the expert system under study here is no exception), while the outcomes of effectiveness trials incorporating the influence of practical barriers should serve as arguments for such a decision. Our results indicate that effectiveness trials are an essential step towards identifying effective interventions that can add significantly to long-term positive changes in health behaviour within the community.

Secondly, when assessing quit attempt rates found within our control group samples, it can be noted that they are higher than what we would expect within a group of smokers not exposed to any intervention. After 12 months, more than $60 \%$ of control group respondents motivated to quit at baseline had made a quit attempt. Including precontemplators, one in two of the total sample had attempted to quit after baseline. This cessation rate is exuberant compared to $14 \%$ of respondents that experienced a 24 hour quit attempt in a national sample in the same time span (STIVORO for a smokefree future 2001b). There are several possible explanations for this difference. Filling out the baseline questionnaire might have been an intervention in itself. In addition, smokers might have provided social desirable answers at follow-up. Also, intermediates might 
have been triggered by the study to offer more assistance in smoking cessation within their daily practice.

Thirdly, the expert system is part of the first wave of computer tailoring interventions: using a written screening instrument and providing paper-based feedback (Skinner et al. 1999). A drawback of these interventions is that between assessment by the screening instrument and providing feedback, a period of time elapses. In this case, respondents would receive the feedback at their home address within seven working days after sending in the written questionnaire. It is possible that values noted in the baseline questionnaire might have changed during this time period. For instance, a smoker might have attempted to quit and failed, resulting in a decrease in self-efficacy to refrain from smoking before receiving the feedback that was based on the self-efficacy level of before the attempt.

Fourthly, the conditions under which the effectiveness trials have been executed were not optimal. In the general introduction (chapter 1), we established that when testing the effectiveness of smoking interventions it is important to limit the possibility of type II error occurrence (Kok 1992); when results show no significant differences when in fact there are. Although the participating community pharmacies and general practices did not provide the expert system to their clients outside those in the experimental condition during the effectiveness trials described in this study, respondents randomised in the control condition were also able to acquire a personalised advice from the expert system. Furthermore, between smoker recruitment in general practices and the follow-up measurement six months later, the Dutch Foundation on Smoking and Health launched a national campaign to aid smokers to quit (STIVORO for a smokefree future 2003b). This campaign took the shape of a free start quitting-kit, including the expert system's baseline questionnaire. Kits could be actively requested from STIVORO, but health care providers were also able to offer these kits in their practice. A total of 21.816 kits were distributed; for instance through a cooperation with a national drug store chain (STIVORO for a smokefree future 2003a). Therefore, respondents in the control group also had access to the expert system, which could partly explain the high quit rate in this group. In addition, the national publicity 
accompanying the campaign could have trigged Dutch smokers in general quit smoking, including those randomised in the control group. A previous Dutch study showed that exposure to a mass media campaign can substantially increase normal cessation rates (Mudde and de Vries 1999). This might account partly for the non-significant differences in smoking behaviour outcomes between experimental and control group.

Lastly, the content of the expert system was tailored on a limited number of concepts that were thought to be essential to reach longer-term abstinence. Further elaboration regarding alterations to the expert system will follow below.

\section{Recommendations}

To increase the expert system's effect, a number of alterations can be applied. Firstly, to ensure the feedback provided is tailored to the smoker's current situation, it should follow immediately after assessment. In this light, web-based interventions offer a promising alternative. Studies are underway to test whether this alteration, which has already been made to the expert system, affects the expert system's effect on smoking outcome.

Secondly, tailored information can be supplied at more than one occasion. Previous studies have shown that offering advice at multiple intervals results in a larger effect on smoking cessation outcomes than a single tailored feedback letter (Borland et al. 2004; Brug et al. 1998; Dijkstra et al. 1998c). By applying ipsative techniques by taking previously expressed determinants and their progression and regression over time into account, smokers can be offered up-to-date feedback for their individual situation, even if the importance of specific concepts have changed. Furthermore, future studies should assess the possibilities to adjust feedback moments to personal preference, difficult moments or lapse/relapse cycles.

Thirdly, although the expert system facilitated a large percentage of 24-hour quit attempts among the respondents in preparation (49-76\%), relatively little abstinence was reached (12-16\%). In other words, the expert system encouraged smokers to attempt to quit, but these attempts were not significantly more successful than attempts without the aid of the expert system. These results suggest that the use of relapse prevention 
strategies was insufficient within the expert system to increase successful longer-term abstinence. Marlatt and Gordon's Relapse Prevention model states that a quitter's coping style in difficult situations determines the risk of relapse (Larimer et al. 1999). Previous research has shown that planning how to cope with these situations before they occur is an effective tool; concepts such as action plans and implementation intentions (which include specific references to time and place of the action) have a significant effect on (smoking) behaviour (De Vries et al. 2006; Gollwitzer 1999; Hilberink et al. 2006; Locke and Latham 2002; Sniehotta et al. 2005). It is therefore recommended to further explore the potential of action plans. Additionally, as active involvement of the individual in formulating their own plans has shown to further improve effect of these plans (Gollwitzer 1999), feedback within the expert system could include an exercise encouraging smokers to make their own plans, in addition to the plans assessed in the baseline questionnaire.

Fourthly, the expert system was based on a limited number of concepts within the I-Change Model, not all identified concepts important for behavioural change were measured and tailored on. For instance, depression has shown to be linked to smoking, smoking cessation and maintenance (Hall et al. 1993). However, we did not assess depression or depressive symptoms, nor did the feedback include information regarding depression. Future studies could assess how to tailor for this individual characteristic. Furthermore, although personality traits are included in the I-Change Model as a predisposing factor, its influence (direct, indirect or both) on behaviour and intention to change in the I-Change Model has not yet been tested as yet. Previous research have provided evidence that possessing specific personality traits, such as neuroticism and anxiety, are related to starting and maintaining to smoke, while other traits such as openness facilitate quitting smoking (Ames et al. 2005; Harakeh et al. 2006; Hooten et al. 2005). It has also been suggested that smokers with different personalities require different interventions (Ames et al. 2005; Hooten et al. 2005). Future studies should test how and if these traits influence responsiveness to specific intervention elements, such as action planning and relaxation exercises. 
Lastly, not all concepts included in expert system were thoroughly measured. For instance, social influence has only been measured regarding smoking behaviour of others in the direct vicinity of the respondents, or modelling. Previous studies have provided evidence that concepts such as social norm and pressure to stop smoking are also of influence of an individual's smoking behavioural change (De Vries et al. 2003; Van den Putte et al. 2005; Viswesvaran and Schmidt 1992). Also, modelling experienced from others than partner and residential children could influence an individual's smoking behaviour. The measurement of action plans was also limited: five preformulated action plans were assessed on dichotomous scale (yes/no). This could have contributed to the small effects found in our study regarding smoking relapse, as described in chapter 8 (Hoving et al. 2006b). Overall, the expert system might benefit from a more extensive elaboration on social influence around the smoker, as well as regarding action plans.

\section{4: Reach of the expert system}

In chapter 6, the impact of active vs. passive recruitment and general practice vs. community pharmacy recruitment setting on acquired samples was compared. In addition, the recruited samples were compared to a Dutch cohort of smokers. Active recruitment was expected to include more smokers not actively interested in a smoking cessation intervention, such as smokers in the pre-contemplation stage. Also, based on previous studies, active recruitment was expected to provide significantly higher revenue (Friebel et al. 2004; McDonald 1999; Sarkin et al. 1998).

All three samples significantly differed from the cohort, which indicates that none of the recruitment methods provided a sample representative of the Dutch adult smoker population. However, these recruitment channels could be applied in combination with other channels, able to reach a larger segment of the Dutch smoking population. In addition, each method resulted in a unique sample of recruited smokers. As expected, active recruitment in the community pharmacies did result in a larger sample, also including more pre-contemplators than passive recruitment in the same setting. Previous research has shown that pre-contemplators are more difficult to recruit 
in comparison those motivated to change their behaviour (Ruggiero et al. 2003). Active recruitment in the community pharmacy $(\mathrm{CP})$ is therefore an appealing strategy for researchers attempting to reach pre-contemplating smokers. However, although active $\mathrm{CP}$ recruitment resulted in the largest sample, it did not significantly outperform $\mathrm{CP}$ passive recruitment. Based on these findings, it would appear that actively recruiting pharmacy staff is not to be preferred over the application of passive recruitment methods in this setting. However, this could be caused by a difference of recruitment intensity between both methods; passive recruitment could be offered at all times, whereas active recruitment depended on the pharmacy staff's cooperation.

Passive GP recruitment provided a sample with significantly more smokers with a lower socio-economic status (measured by education level) than passive CP recruitment. This sample was also the least different from the cohort sample. Previous research has shown that a lower socio-economic status predicts smoking onset and smokers with a lower SES are less likely to quit smoking than their high SES counterparts (Willemsen et al. 2002). Furthermore, these smokers are also less likely to be included in smoking cessation clinical trials (Bolen et al. 2006); the general practice could therefore be a recruitment channel worth exploring when attempting to include lower SES smokers.

\section{Recommendations}

Unexpectedly, active recruitment did not significantly reach a larger sample of smokers. However, based on results described in chapter 5, it is clear pharmacy assistants were unlikely to consistently recruit in an active manner throughout the recruitment period. Future studies should aim to compare the impact of a passive compared to a truly active recruitment strategy, as well as investigate the possibilities and preconditions to implement such an active strategy in community pharmacy and general practice setting.

A research topic of interest could be the actual interaction between recruiter (i.e. staff member) and recruited (i.e. smoking client). Recruiter characteristics (i.e. gender, age, personality) and cognitive factors expressed by the recruiter (e.g. attitude, selfefficacy) are likely to influence recruiting dynamic processes. The I-Change Model 
suggests that socio-cultural factors such as ethnicity can influence the evaluation of channel and source of behaviour-related messages. Furthermore, a review focusing on recruitment and retention strategies of minority research participants suggests that ethnically matched recruiters might increase recruitment rate (Yancey et al. 2006). However, they also suggest that its effect is likely to be influenced by the experience and know-how of the recruiter. Future studies should take a recruiter's experience and training into account, as well as test whether the recruitment strategies under study can reach smokers from minority groups, which have shown to be difficult to reach (Baquet et al. 2006; Lee et al. 1997; Yancey et al. 2006).

Moreover, limited attention has been focused on information factors, such as channel characteristics, and how they influence the motivational determinants. In the IChange Model, information factors only influence awareness factors, such as risk perception. However, the results in chapter 6 indicate the channel characteristics may also affect motivational determinants. The I-Change Model stipulates that this relation is mediated by awareness factors, such as risk perception. Further research is necessary tot test this assumption, as well as the extent of the assumed mediation (complete or partly).

\section{5: Predictors of stage transition}

Chapter 7 summarizes the results of a study regarding forward and backward transitions through the stage of change paradigm in a Dutch sample of adult smokers. Based on presumptions of the I-Change Model and results from previous studies (De Vries and Backbier 1994; De Vries and Mudde 1998; De Vries et al. 1998), we expected to cross-sectionally replicate the $\varnothing$ pattern (pre-contemplators perceive less pros of quitting smoking than contemplators, who are less confident to be able to quit than preparers). Furthermore, we expected a forward transition from pre-contemplation (towards action) to be predicted by perceiving more pros of cessation, a backward transition from contemplation (to pre-contemplation) to be predicted by perceiving less pros of smoking cessation and a forward transition from preparation (to action) to be predicted by a higher self-efficacy to quit smoking. 
Cross-sectional results showed a confirmation of the $\varnothing$ pattern. Smokers were also more likely to transition to an adjacent stage than to skip one or more stages. Longitudinal tests partly confirmed results from a previous Dutch study (De Vries and Mudde 1998). However, perceiving less advantages of quitting smoking did not predict backward transitions from contemplation. Our results show a single significant predictor for a backward transition from contemplation after three months was gender; males were more likely to regress to pre-contemplation than females. No predictors for this transition were identified at the 12-month follow-up. A study by De Vries \& Mudde identified perceiving fewer pros as a predictor of this transition (De Vries and Mudde 1998), but another Dutch study did not find any significant predictors (Dijkstra et al. 2003). Both studies, as well as our own study, included similar concepts in the analyses. Furthermore, a recent match-mismatch study showed contemplators were significantly more likely to experience a forward transition when being exposed to an intervention aiming to diminish perceived cons of cessation. However, cross-sectional analyses in that study showed no significant difference between pre-contemplators, contemplators and preparers on perceived cons, as did our analyses. It is possible that between baseline assessment and follow-up an alteration in perceived cons occurred, necessary to make the transition but non-existent at baseline.

Self-efficacy was a significant predictor of all forward transitions, including from pre-contemplation. Moreover, the odds ratio predicting forward transition from pre-contemplation and preparation were identical $(\mathrm{OR}=1.48)$. This seems to support the Social Cognitive Theory, stating that self-efficacy is thought to be important regardless of the strength of the intention to quit (Bandura et al. 1977). Recent research on the stage of change paradigm has also shown that self-efficacy predicted all forward transitions with regard to fruit and vegetable consumption (De Vet et al. 2005). In our study, backward transitions were mainly predicted by demographic variables and previous quitting behaviour, not by cognitive determinants. Predictors of forward transitions included in the analyses explained only $1-14 \%$ of the variance, whereas predictors of backward transitions explained not more than $0-8 \%$ of the variance. 
Assignment to a specific stage at one point in time is assumed to be predictive of smoking cessation activity measured at a later point in time with smokers closer to the action stage being more active (DiClemente et al. 1991). However, chapter 7 provides findings that suggest that smokers in the different stages were just as likely to be in the action stage at follow-up. When performing Tukey HSD contrasts within our sample randomised into the control group only, preparers were significantly more likely to attempt to quit and reach continued abstinence three months after baseline. Nevertheless, no significant differences were observed between pre-contemplators and contemplators. These findings are somewhat contradictive to a recent match-mismatch study assessing the predictive quality of the stage of change concept on quitting and abstinence measures (Dijkstra et al. 2006). This study indicated that after two months, stage at baseline significantly predicted the likelihood of a quit attempt and point prevalence abstinence. However, all respondents received either a matched or mismatched intervention during these two months, but no control group was included. It is therefore unclear whether mismatched interventions might have had a negative influence on smoking behaviour outcome measures.

\section{Recommendations}

The results from chapter 7 provide limited support for the usability of the stage of change paradigm to measure intention and predict behaviour change. These findings and those of other previous studies suggest a re-assessment of the inclusion of the stage of change concept as an indicator of intention to change behaviour with regard to smoking. It might therefore be prudent to begin the search for a more accurate measure. As the stage of change paradigm is a practical approach, understandable for lay or semilay people (such as health professionals) and highly popular with health practice, a stage approach should not be abandoned. However, the distinctions between the different stages should not be based on the time period in which a behaviour change is intended to occur, but on strength of the intention to facilitate that change. The Precaution Adoption Process Model (Weinstein 1988) might provide a good starting point. This stage model consists of seven states: unawareness of a problem, awareness but not 
personally engaged, engaged and deciding what to do, planning to act but not yet having acted, having decided not to act, acting and maintenance. The stages are defined in terms of beliefs individuals have regarding the health behaviour, not in periods of time in which they are intending to change their behaviour. More research is necessary to ascertain whether aspects of this model might be incorporated in the I-Change Model and to what extent.

\section{6: Predictors of relapse}

In chapter 8 , predictors of relapse after a quit attempt were identified in a sample of adult smokers at baseline, followed up after three months. We hypothesized that predictors of relapse would differ for male and female adult respondents. Furthermore, we expected that in addition to cognitive factors, intending to use certain action plans would have a significant protective effect against relapse.

In our study, women with low self-efficacy expectations (regarding being able not to smoke when confronted with negative outcomes of not smoking, such as weight gain and stress) were more likely to relapse. This finding is in line with previous studies, showing expected or manifested negative affect when refraining from smoking, (such as post-cessation weight gain and stress) is of particular importance for women (Copeland et al. 2006; Perkins 2001; Wetter et al. 1999). Furthermore, women reporting a higher addiction level were more likely to restart smoking. Again, this finding is in line with previous findings that women are more likely to be higher dependent on nicotine (Kandel and Chen 2000) and smokers with a higher nicotine dependence are more likely to return to smoking after a quit attempt (Ockene et al. 2000). Nevertheless, other studies have found that women are less dependent on nicotine using biological measures such as serum nicotine (Wetter et al. 1999). However, although physical dependence might be less, women's emotional dependence on tobacco smoking might be higher. This emotional dependence could be related to perceived self-efficacy and coping skills.

Having a smoking partner was a predictor of relapse for men only. This is in contradiction with the assumption that women are more socially oriented and its influence would therefore be stronger on the female's smoking behaviour (Centers for 
Disease Control and Prevention 2001). However, a very recent study showed no effect of gender, although the influence of the partner on smoking behaviour was more pronounced in women (Manchon Walsh et al. 2007). It is possible that for men, smoking can serve as a way to communicate non-verbally or share an intimate moment with their spouse (Rohrbaugh et al. 2001).

Results presented in chapter 7 also indicated a limited predictive quality of two action plans in relation to relapse; removing all smoking materials from the home and quitting immediately were both borderline predictors of relapse in our sample. This is only partly in line with our assumption that intending to perform certain action plans would be a protective factor against relapse. However, besides including a very limited set of action plans, we limited measurements to the intention to use action plans, not the actual use of these plans. Furthermore, it is possible that relying on one action plan might result in an under-estimation of the degree of difficulty related to a quit attempt.

\section{Recommendations}

A number of gender-related predictors of relapse were identified. Interventions might be able to change female smoker's dependence level by increasing self-efficacy and improving skills to cope with negative emotions and thereby improve cessation success rates. In addition, it might be especially prudent for male smokers to also include their partners in smoking cessation interventions.

Also, the action plans assessed in this study were limited to five plans, which were formulated in the questionnaire. Research has shown that inclusion of a trigger situation in the plan (if this happens, I will do that) and self-formulated plans improve its effect (Gollwitzer 1999). Based on the limited effect of action plans in our study, smokers attempting to quit should be encouraged to adopt a more elaborate relapse prevention strategy. Studies are currently underway to assess the most effective formulation of action plans regarding behaviour change. 


\section{Methodological issues}

Trials

During both study periods (community pharmacy and general practice), the baseline questionnaires had been available to individuals and health professionals by ordering the questionnaire from the Dutch Foundation on Smoking and Health. In 2001, 11.595 baseline questionnaires were distributed within the Netherlands (STIVORO for a smokefree future 2001a). In 2003, 8.481 tailored advice letters were distributed, in addition to more than 8000 respondents who had used the web-based version of the expert system (STIVORO for a smokefree future 2003a). As mentioned before, during the study in general practices, a national campaign including the expert system was launched and received much attention. Although we did assess whether people from the control group used cessation aids if they had attempted to quit between baseline and follow-up, but these questions did not specifically pertain to the expert system. Although none of the respondents at follow-up indicated that they had used the expert system as a cessation tool during the trial period, it is possible that these respondents did not view the expert system as a cessation tool. Many respondents indicated to have used pharmacotherapy (20-25\%), but only a few indicated to have used behavioural group therapy $(0-1 \%)$. It is therefore possible that respondents in the control condition also received the tailored advice.

Furthermore, one of the follow-up periods from the community pharmacy study (three and 12 months from baseline) matched the follow-up period in the general practice (six months after baseline). It was therefore not possible to compare both samples on follow-up measurements. Subsequently, conclusions concerning the effectiveness of the expert system in community pharmacy could not be compared against the effectiveness in general practice setting. The original design did include identical follow-up periods in both settings to facilitate comparison in effectiveness. However, due to administrative errors within the independent data processing centre, the general practice study had to be abandoned and retried, constraining the time schedule and leaving only room for one follow-up. This is unfortunate; the comparison 
would have been of high interest, as the community pharmacy has only been utilized sparingly as intervention channel for smoking cessation interventions.

Based on the methodological issues encountered, we would recommend a retrial to test the effectiveness of the expert system. However, new developments regarding the expert system have been observed. Currently, the Dutch Foundation on Smoking and Health only offers the expert system through their website (STIVORO for a smokefree future 2006). Smokers are now asked to fill out the baseline questionnaire online, after which they receive a tailored feedback letter in their e-mail inbox the same day. Since 2005 a study is underway dismantling the expert system to increase its efficacy (Te Poel, unpublished). Within three studies, the expert system will be complemented by an add-on intervention, intensifying tailored feedback on the subjects of action plans, perceived social support and emotions related to smoking and relapse. Preferably, if proven to be effective, the improved expert system should again be tested under 'effectiveness' circumstances. Final results of the dismantling study are expected in 2009.

Assessment smoking behaviour

Initiating a quit attempt for at least 24 hours, abstinence for at least 7 days at follow-up (point prevalence) and abstinence during the entire trial period between baseline and respective follow-ups (continuous abstinence) have been used as behavioural outcome measures in this dissertation. These measures have been used frequently in smoking cessation research. However, some issues have not been addressed in the current studies that could increase the validity of the outcome measures. Future studies should incorporate these alterations.

Firstly, it should be considered whether the occurrence of a quit attempt is a valid outcome measure to assess an intervention's effectiveness. After all, a smoker only truly benefits from cessation if it is permanent. As described before, only $7 \%$ of quit attempts is estimated to be successful (Baillie et al. 1995). In our study described in chapter 8 , we registered a relapse rate of $73 \%$ three months after baseline. These analyses were corrected for randomisation category, which did not reach significance in any 
model tested. In other words, randomisation in the experimental group (i.e. receiving the intervention) did not appear to protect against relapse. In light of these findings, we can conclude that quit attempt has limited value when aiming to describe the long-term success of an intervention. An intervention effect over considerable time should therefore be tested using solely abstinence measures, such as point prevalence abstinence and continued abstinence.

Secondly, we used a conservative measure to assess continuous abstinence; respondents who had smoked even a puff were considered not to be abstinent continuously. However, smokers can experience minor setbacks in smoking behaviour (lapses), such as smoking one cigarette or smoking on one occasion, after which they are again committed to quitting. The continuous abstinence measure used in our studies did not allow for this option. Although a number of studies have shown that a lapse is a major predictor of full-blown relapse (Garvey and Bliss 1992; Nides et al. 1995; Ockene et al. 2000), the distinction between lapse and relapse is an important issue. In relation to this, previous studies have shown that most relapse will occur within days after the initial quit attempt (Hughes et al. 2004a). In our study, continued abstinence was measured starting from baseline, not allowing the intervention received to take effect. Two main improvements to the outcome measure could have been implemented: application of a grace period and allowing for a limited number of smoked cigarettes within the measure. A grace period is a short period directly after administrating the intervention in which lapse and relapse are not considered as treatment failure, providing the smoker with an opportunity to quit smoking through a trial-and-error approach. The use of a two-week grace period is currently recommended by a number of research groups (Hughes et al. 2003; West et al. 2005).

Thirdly, at follow-up, respondents were asked to retrospectively describe their smoking behaviour in terms of quitting, abstinence and relapse since the baseline measurement. Time periods covered were three, six and 12 months. Although it is unlikely that respondents forgot longer-term changes in their smoking (such as being abstinent for three months), it is possible that smokers were not able to accurately 
remember smaller changes, such as a short-term quit attempt (e.g. 1-2 days) and lapsing behaviour (having smoked one cigarette, but otherwise abstinent).

Fourthly, when assessing smoking behaviour outcomes, we relied on selfreported abstinence without biochemical validation. It has been argued that the reliability of self-reported outcomes can be influenced by a number of threats, such as social desirability. Smokers could therefore alter their responses to questions concerning outcome measures as a means to impress or provide answer they think are expected of them (Green and Lewis 1986). However, previous studies have shown that self-reported smoking status among adult smokers is fairly accurate when there is no direct contact between researchers and respondents and there is no extra gain by providing specific answers (Glasgow et al. 1993; Patrick et al. 1994; Velicer et al. 1992). Also, as respondents resided throughout the Netherlands, it was logistically difficult to systematically validate their self-reported smoking status, as validation is only relevant within a limited time after the smoking occurs (Stevens and Munoz 2004). Moreover, some validation methods do not account for the use of Nicotine Replacement Therapy (a smoker quitting with the help NRT would then be classified as a current smoker), are too invasive or need medically trained assistance (Stevens and Munoz 2004). Furthermore, the announcement of a biochemical validation could increase non-response and dropout among the respondents, leaving a highly selected group of respondents.

\section{Practical issues}

\section{Recruitment of intermediates}

During recruitment of community pharmacies and general practices for our study, a number of methods were applied. Pharmacists that had shown interest in smoking cessation methods in the past through the Scientific Institute Dutch Pharmacists (WinAp) or by requesting materials from the Dutch Foundation on Smoking and Health (STIVORO for a smokefree future) were approached first. This did not result in a sufficient number of pharmacies. Following this, Dutch pharmacy addresses were identified from the telephone registries and a written invitation to participate was sent. No distinction was made regarding location, all known pharmacies were invited. The 
invitation was mailed to the pharmacies, with a leaflet containing information on the research project and activities expected from participating community pharmacies. Subsequently, a telephone call was made to the pharmacist by a project employee and initial interest to participate was assessed. Pharmacies could also indicate their interest by returning an interest form to the project team, after which telephonic contact was established. During the telephone call, a meeting was set up for a project employee to come to the pharmacy, bring the project materials and introduce the projects and the activities to be carried out to the pharmacy assistants. We had expected that the pharmacist would discuss with the pharmacy assistants or at least inform them of the pharmacy's participation in the project, as most tasks were to be carried out by these assistants. However, in the majority of pharmacies we visited to introduce the project, the assistants were not aware of their pharmacist's agreement to participate. On a few occasions, this resulted in an unwillingness to perform the tasks by a part of the assistants, but in only two pharmacies outright resistance from the entire assistant group was observed. These pharmacies were subsequently excluded from the project. Given these responses, recruitment of pharmacies could have been improved by including pharmacy assistants as a focus of our recruitment strategies. Possible alterations in the recruitment strategy include emphasising the importance of discussion with the assistants to the pharmacist and providing time to do so, organising meetings with pharmacy staff only to inform while recruiting in a later stage and directly focusing recruitment on pharmacy assistants.

General practices were recruited with the aid of two District General Practitioner's Associations (DHVs). Invitations to participate were sent to all DHVregistered GPs and printed on DHV stationary. We hypothesized that as GPs receive large amounts of mail in general and invitations to participate in research in particular, a mailing via the DHV would stand out. The invitation, together with the same leaflet as used during pharmacy recruitment but adjusted for the passive general practice approach and a short questionnaire, was followed by a recruitment call from a project employee. During these calls, it became clear that many GPs had not read the invitation or did not know of its existence. Often, the contacting project employee had to 
summarize the intervention and repeat what was expected of participating general practices. It appears that our strategy of including District General Practitioner's Associations in our recruitment effort did not have the desired effect. Possible improvements regarding the recruitment of general practices include recruitment directed at practice assistants or practice nurses and inclusion of GP opinion leaders in recruitment. Furthermore, alternative recruitment channels should be explored, such as presentations at GP-related conferences or meetings. Telephone recruitment directed at GPs does not seem to be a good alternative, as in our experience during this study, GPs were also difficult to contact by phone during office hours. Recently, the DHV structure (including 21 DHVs) in the Netherlands was abandoned, replaced by 23 regional GP circles (Landelijke Huisartsen Vereniging 2006). Although these circles might also prove useful as a recruiting channel for general practices, providing GPs with written information sent by post appears not to be the preferred strategy. Strategy improvements mentioned before can possibly be integrated in this channel.

\section{Drop-out of smokers}

Through community pharmacies, at total of 925 smokers returned their baseline questionnaire. In general practices, a total of 673 smokers was recruited, of which 10 did not adhere to the inclusion criteria set for these studies ( 9 were 17 years of age or younger and one respondent did not smoke at baseline). Table 9.1 provides an overview of the dropout in our study samples.

Table 9.1.

Study sample dropout.

\begin{tabular}{llll}
\hline & $\begin{array}{l}\text { Pharmacy } \\
1 \text { st follow-up (3 months) }\end{array}$ & $\begin{array}{l}\text { Pharmacy } \\
2^{\text {nd }} \text { follow-up (12 months) }\end{array}$ & $\begin{array}{l}\text { General practice } \\
\text { follow-up (6 months) }\end{array}$ \\
\hline $\begin{array}{l}\text { Drop- } \\
\text { out }\end{array}$ & 246 of $925(26.6 \%)$ & 264 of $925(28.5 \%)$ & 140 of $663(21 \%)$ \\
\hline
\end{tabular}

The percentage of respondents that dropped out is comparable to study testing single computer-tailored interventions against no condition control groups, where drop-out 
was recorded to be around 30\% (Dijkstra et al. 1998d; Spittaels et al. 2007). Nevertheless, it is possible that dropout resulted in selection (Green and Lewis 1986), as respondents failed to quit or maintain a non-smoking status could have been more likely to refuse further participation. However, by conducting intention-to-treat analyses to test the effect of the intervention, bias due to drop-out was limited.

From the pharmacy-recruited sample, $18.9 \%$ of randomised respondents were never reached for follow-up. This was mostly due to missing or wrong contact information, which the smoker was asked to fill out on the baseline questionnaire. Future studies might benefit from application of strategies to improve registration of respondents. Web-based programmes can deny access to the randomisation until all contact details are registered. Involving pre-existing address details lists, for instance patient list from general practices, could also limit hiatuses in contact details.

Some respondents also dropped out because they were not willing to participate in follow-ups. Previous studies have shown that offering monetary incentives to remain in a trial has a positive effect on retention (Edwards et al. 2002; Martinson et al. 2000); this measure could be applied in future effectiveness studies. A study regarding response rates among physicians indicates that smaller incentives in sequential questionnaires may be more cost-effective that one large incentive (Halpern et al. 2002). However, further research is necessary to determine which is most effective among adult smokers.

\section{General conclusions}

Based on the results from the analyses described in this dissertation, the following main conclusions can be drawn:

$>$ Recruitment of adult smokers to participate in a smoking cessation randomised controlled trial is feasible in general practice and community pharmacy, but participating staff need to create a sense of ownership and responsibility for the recruitment and subsequent expert system. 
> In order to improve Dutch general practices and community pharmacies' involvement in smoking cessation activities, providing smoking cessation advice to specific risk groups and smokers actively looking to quit, in combination with short action periods to approach the general public should be employed.

$>$ Implementation of the expert system in Dutch general practice and community pharmacy is feasible, but a number of barriers need to be addressed before national implementation can be accomplished. Self-efficacy to offer smoking cessation advice and social influences from direct colleagues needs to be addressed, for instance through providing continues training and extensive feedback on their actions. Practice nurses should be included in smoking cessation activities in general practice.

$>$ The expert system's effect on smoking behaviour was limited to making quit attempts in the year after baseline. These results can not be attributed to the intervention, as the expert system was nationally distributed and could be obtained by respondents randomized in the control condition. The expert system could benefit from including a relapse prevention programme, integrated in multiple tailoring moments over time.

$>$ It is questionable that the stage of change paradigm is an effective tool to base smoking cessation interventions on. Results described in this study are in line with numerous critiques on this paradigm. Self-efficacy enhancing information should be provided to all smokers, regardless of their current stage. Future research should investigate stage-based alternatives that can be utilized in tailored interventions. 



\section{References}

Ames SC, Vickers KS, Decker PA, Patten CA, Colligan RC, Vargas-Chanes D, Schroeder D and Offord KP. Select Minnesota Multiphasic Personality Inventory (MMPI) scales as predictors of tobacco abstinence following treatment for nicotine dependence. 2005. Psychology and Health 20(3): 331-351.

Anon. The health consequences of smoking: a report of the Surgeon General. 2004.

Anon. Zorg om ICT. Internationaal vergelijkend onderzoek naar ICT-gebruik in the gezondheidszorg [Care about ICT. International comparison of ICT-use in health care]. 2001.

Anon. The impact of passive smoking on public health. 2003a.

Anon. Geneeskundig Adresboek [Medical Directory]. 2003b. Bohn Stafleu Van Loghum, Houten.

Anon. Nationaal Kompas Volksgezondheid [National Compass Public Health]. 2005.

Ausems M, Mesters I, van Breukelen G and de Vries H. Short-term effects of a randomized computer-based out-of-school smoking prevention trial aimed at elementary schoolchildren. 2002. Prev Med 34(6): 581-589.

Baillie AJ, Mattick RP and Hall W. Quitting smoking: estimation by meta-analysis of the rate of unaided smoking cessation. 1995. Aust J Public Health 19(2): 129-131.

Bakker MJ, Mullen PD, de Vries H and van Breukelen G. Feasibility of implementation of a Dutch smoking cessation and relapse prevention protocol for pregnant women. 2003. Patient Educ Couns 49(1): 35-43.

Bandura A, Adams NE and Beyer J. Cognitive processes mediating behavioral change. 1977. J Pers Soc Psychol 35(3): 125-139.

Bandura A. The anatomy of stages of change. 1997. Am J Health Promot 12(1): 8-10.

Baquet CR, Commiskey P, Mullins CD and Mishra SI. Recruitment and participation in clinical trials: Socio-demographic, rurual/urban, and health care access predictors. 2006. Cancer Detect Prev 30(1): 24-33.

Bayram C, Britt H, Kelly Z and Valenti L. Male consultations in general practice in Australia 1999-2000. 2003. 
Berger K, Eickhoff C and Schulz M. Counselling quality in community pharmacies: implementation of the pseudo customer methodology in Germany. 2005. J Clin Pharm Therap 30(1): 45-57.

Bero LA, Grilli R, Grimshaw JM, Harvey E, Oxman AD and Thomson MA. Closing the gap between research and practice: an overview of systematic reviews of interventions to promote the implementation of research findings. The Cochrane Effective Practice and Organization of Care Review Group. 1998. BMJ 317(7156): 465-468.

Blenkinsopp A, Anderson C and Armstrong M. Systematic review of the effectiveness of community pharmacy-based interventions to reduce risk behaviours and risk factors for coronary heart disease. 2003. J Public Health 25(2): 144-153.

Bolen S, Tilburt J, Baffi C, Gary TL, Powe N, Howerton M, Ford J, Lai G, Wilson R and Bass E. Defining success in recruitment of underrepresented populations to cancer clinical trials. 2006. Cancer 106(6): 1197-1204.

Bolman C and de Vries H. Psycho-social determinants and motivational phases in smoking behavior of cardiac inpatients. 1998. Prev Med 27(5 Pt 1): 738-747.

Bolman C, de Vries H and Mesters I. Factors determining cardiac nurses' intentions to continue using a smoking cessation protocol. 2002. Heart Lung 31(1): 15-24.

Borland R, Balmford J and Hunt D. The effectiveness of personally tailored computergenerated advice letters for smoking cessation. 2004. Addiction 99(3): 369-377.

Borrelli B, Spring B, Niaura R, Hitsman B and Papandonatos G. Influences of gender and weight gain on short-term relapse to smoking in a cessation trial. 2001.

J Consult Clin Psychol 69(3): 511-515.

Bruce N and Burnett S. Prevention of lifestyle-related disease: general practitioners' views about their role, effectiveness and resources. 1991. Fam Pract 8(4): 373377.

Brug J, Steenhuis I, van Assema P and de Vries H. The impact of a computer-tailored nutrition intervention. 1996. Prev Med 25(3): 236-242. 
Brug J, Glanz K, van Assema P, Kok G and van Breukelen GJP. The impact of computertailored feedback and iterative feedback on fat, fruit, and vegetable intake. 1998. Health Educ Behav 25(4): 517-531.

Brug J, Campbell $\mathrm{M}$ and van Assema P. The application and impact of computergenerated personalized nutrition education: A review of the literature. 1999. Patient Educ Couns 36(2): 145-156.

Bull FC, Kreuter MW and Scharff DP. Effects of tailored, personalized and general health messages on physical activity. 1999. Patient Educ Couns 36(2): 181-192.

Cavill $\mathrm{N}$ and Bauman A. Changing the way people think about health-enhancing physical activity: do mass media campaigns have a role? 2004. Journal of Sports Sciences 22(8): 771-790.

Centers for Disease Control and Prevention. Women and smoking: a report of the Surgeon General. 2001.

Centers for Disease Control and Prevention. The health consequences of smoking: a report of the Surgeon General. 2004.

Centraal Bureau voor de Statistiek. Vademecum gezondheidsstatistiek 2003 [Vademecum health statistics 2003]. 2003.

Centraal Bureau voor de Statistiek. Gerapporteerde gezondheid en leefstijl [Reported health and lifestyle]. 2006. http:// statline.cbs.nl

Coleman $\mathrm{T}$ and Wilson A. Factors associated with the provision of anti-smoking advice by general practitioners. 1999. Br J Gen Pract 49(444): 557-558.

Coleman T, Murphy E and Cheater F. Factors influencing discussion of smoking between general practitioners and patients who smoke: a qualitative study. 2000. Br J Gen Pract 50(452): 207-210.

Concato J, Shah N and Horwitz RI. Randomized, Controlled Trials, Observational Studies, and the Hierarchy of Research Designs. 2000. N Engl J Med 342(25): 1887-1892.

Conrad KM, Flay BR and Hill D. Why children start smoking cigarettes: Predictors of onset. 1992. Br J Addict 87 (12): 1711-1724. 
Copeland AL, Martin PD, Geiselman PJ, Rash CJ and Kendzor DE. Smoking cessation for weight-concerned women: Group vs. individually tailored, dietary, and weightcontrol follow-up sessions. 2006. Addictive Behav 31(1): 115-127.

Dar R and Frenk H. Nicotine addiction: Fact or theory? 2002. Addiction 10(3): 219-224.

De Nooijer J, Lechner L and de Vries H. Tailored versus general information on early detection of cancer: a comparison of the reactions of Dutch adults and the impact on attitudes and behaviors. 2002. Health Educ Res 17(2): 239-252.

De Nooijer J, Lechner L and De Vries H. Social psychological correlates of paying attention to cancer symptoms and seeking medical help. 2003. Soc Sci Med 56(5): 915-920.

De Vet E, De Nooijer J, De Vries NK and Brug J. Determinants of forward stage transition from precontemplation and contemplation for fruit consumption. 2005. Am J Health Promot 19(4): 278-285.

De Vries H, Dijkstra M and Kuhlman P. Self-efficacy: The third factor besides attitude and subjective norm as a predictor of behavioural intentions. 1988. Health Educ Res 3(3): 273-282.

De Vries H and Backbier E. Self-efficacy as an important determinant of quitting among pregnant women who smoke: the phi-pattern. 1994. Prev Med 23(2): 167-174.

De Vries H. Planning and evaluating health promotion. In: D Scott and R Weston. Evaluating Health Promotion. 1998. Stanley Thornes Ltd, Cheltenham.

De Vries $\mathrm{H}$ and Mudde AN. Predicting Stage Transitions for Smoking Cessation Applying the Attitude-Social Influence-Efficacy Model. 1998. Psychology and Health 13(2): 369-385.

De Vries H, Mudde AN, Dijkstra A and Willemsen MC. Differential beliefs, perceived social influences, and self-efficacy expectations among smokers in various motivational phases. 1998. Prev Med 27(5): 681-689.

De Vries $\mathrm{H}$ and Brug J. Computer-tailored interventions motivating people to adopt health promoting behaviours: Introduction to a new approach. 1999. Patient Educ Couns 36(2): 99-195. 
De Vries H, Mudde A and Dijkstra A. The Attitude-Social Influence-Efficacy Model applied to the prediction of motivational transitions in the process of smoking cessation. In: P Norman and C Abraham. Understanding and Changing Health Behaviour: From Health Beliefs to Self Regulation. 2000. Harwood Academic Publishers, Amsterdam.

De Vries H, Mudde A, Leijs I, Charlton A, Vartiainen E, Buijs G, Clemente MP, Storm H, Gonzalez Navarro A, Nebot M, Prins T and Kremers S. The European Smoking prevention Framework Approach (EFSA): an example of integral prevention. 2003. Health Educ Res 18(5): 611-626.

De Vries H, Mesters I, van 't Riet J, Willems K and Reubsaet A. Motives of Belgian adolescents for using suncreen: The role of action plans. 2006. Cancer Epidemiol Biomarkers Prev 15(7): 1360-1366.

DiClemente CC, Prochaska JO, Fairhurst SK, Velicer WF, Velasquez MM and Rossi JS. The process of smoking cessation: an analysis of precontemplation, contemplation, and preparation stages of change. 1991. J Consult Clin Psychol 59(2): 295-304.

Dijkstra A, de Vries H and Bakker M. Pros and cons of quitting, self-efficacy, and the stages of change in smoking cessation. 1996. J Consult Clin Psychol 64(4): 758763.

Dijkstra A, Bakker M and De Vries H. Subtypes within a sample of precontemplating smokers: a preliminary extension of the stages of change. 1997. Addictive Behaviors 22(3): 327-337.

Dijkstra A, De Vries H and Roijackers J. Computerized tailored feedback to change cognitive determinants of smoking: A Dutch field experiment. 1998a. Health Educ Res 13(2): 197-206.

Dijkstra A, De Vries H and Roijackers J. Long-term effectiveness of computer-generated tailored feedback in smoking cessation. 1998b. Health Educ Res 13(2): 207-214.

Dijkstra A, De Vries H, Roijackers J and van Breukelen G. Tailoring information to enhance quitting in smokers with low motivation to quit: Three basic efficacy questions. 1998c. Health Psychol 17(6): 513-519. 
Dijkstra A, De Vries H, Roijackers J and van Breukelen G. Tailored interventions to communicate stage-matched information to smokers in different motivational stages. 1998d. J Consult Clin Psychol 66(3): 549-557.

Dijkstra A and de Vries $\mathrm{H}$. The development of computer-generated tailored interventions. 1999. Patient Educ Couns 36(2): 193-203.

Dijkstra A and Borland R. Residual Outcome Expectations and Relapse in Ex-smokers. 2003. Health Psychology 22(4): 340-346.

Dijkstra A, Tromp D and Conijn B. Stage-specific psychological determinants of stage transition. 2003. Br J Health Psychol 8(4): 423-437.

Dijkstra A, Conijn B and de Vries H. A match-mismatch test of a stage model of behavior change in tobacco smoking. 2006. Addiction 101(7): 1035-1043.

Doll $\mathrm{R}$ and Hill AB. Lung cancer and other causes of death in relation to smoking; a second report on the mortality of British doctors. 1956. BMJ 2(5001): 1071-1081.

Doll R, Peto R, Boreham J and Sutherland I. Mortality in relation to smoking: 50 years' observations on male British doctors. 2004. BMJ 328(7455): 1519.

Edwards P, Roberts I, Clarke M, DiGuiseppi C, Pratap S, Wentz R and Kwan I. Increasing response rates to postal questionnaires: systematic review. 2002. BMJ 324(7347): 1183.

Etter J-F and Perneger TV. Effectiveness of a Computer-Tailored Smoking Cessation Program: A Randomized Trial. 2001. Arch Intern Med 161(21): 2596-2601.

Etter J-F and Sutton SR. Assessing 'stage of change' in current and former smokers. 2002. Addiction 97(9): 1171-1182.

Farkas AJ, Pierce JP, Zhu SH, Rosbrook B, Gilpin EA, Berry C and Kaplan RM. Addiction versus stages of change models in predicting smoking cessation. 1996. Addiction 91(9): 1271-1280.

Fishbein M and Ajzen I. Belief, attitude, intention and behavior: An introduction to theory and research. 1975. Addison-Wesley, Reading.

Flay BR and Phil D. Efficacy and effectiveness trials (and other phases of research) in the development of health promotion programs. 1986. Prev Med 15(5): 451-474. 
Flay BR. Mass media and smoking cessation: a critical review. 1987. Am J Public Health 77(2): 153-160.

Friebel TM, Beutler RA, Lee SM, Bernhardt BA, Helzlsouer KJ and Griffin CA. Active recruitment increased enrollment in a heridiary cancer registry. 2004. J Clin Epidemiol 57(11): 1172-1176.

Gallant MP and Dorn GP. Gender and race differences in the predictors of daily health practices among older adults. 2001. Health Educ Res 16(1): 21-31.

Garvey AJ and Bliss RE. Predictors of smoking relapse among self-quitters: a report from the normative aging study. 1992. Addictive Behaviors 17(4): 367-377.

Geraets JJXR, de Groot IJM, Goossens MEJB, de Bruijn CPC, de Bie RA, van den Heuvel WJA and Dinant G. Comparison of two recruitment strategies for patients with chronic shoulder complaints. 2006. Br J Gen Pract 56(523): 127-133.

Glasgow RE, Mullooly JP, Vogt TM, Stevens VJ, Lichtenstein E, Hollis JF, Lando HA, Severson $\mathrm{HH}$, Pearson KA and Vogt MR. Biochemical validation of smoking status: pros, cons, and data from four low-intensity intervention trials. 1993. Addict Behav 18(5): 511-527.

Glasgow RE, Lichtenstein E and Marcus AC. Why don't we see more translation of health promotion Research to Practice? Rethinking the Efficacy-to-Effectiveness Transition. 2003. Am J Public Health 93(8): 1261-1267.

Gollwitzer PM. Implementation intentions. Strong effects of simple plans. 1999. Am Psychol 54(7): 493-503.

Green LW and Lewis FM. Measurement and evaluation in health education and health promotion. 1986. Mayfield Publishing Company, Palo Alto.

Green LW and Kreuter MW. Applications of PRECEDE-PROCEED in Community Settings. In: Health Promotion Planning. An educational and environmental approach. 1991. Mayfield Publishing Company, Mountain View.

Hall SM, Munoz RF, Reus VI and Sees KL. Nicotine, negative affect, and depression. 1993. J Consult Clin Psychol 61(5): 761-767. 
Halpern SD, Ubel PA, Berlin JA and Asch DA. Randomized trial of 5 dollars versus 10 dollars monetary incentives, envelope size, and candy to increase physician response rates to mailed questionnaires. 2002. Med Care 40(9): 834-839.

Harakeh Z, Scholte RHJ, De Vries H and Engels RCME. Association between personality and adolescent smoking. 2006. Addict Behav 31(2): 232-245.

Harris KJ, Ahluwalia JS, Catley D, Okuyemi KS, Mayo MS and Resnicow K. Successful recruitment of minorities into clinical trials: the Kick It as Swope project. 2003. Nic Tob Res 5(4): 575-584.

Heatherton TF, Kozlowski LT, Frecker RC and Fagerstrom KO. The Fagerstrom Test for Nicotine Dependence: a revision of the Fagerstrom Tolerance Questionnaire. 1991. Br J Addict 86(9): 1119-1127.

Helgason AR and Lund KE. General practitioners' perceived barriers to smoking cessation - results from four Nordic countries. 2002. Scand J Public Health 30(2): 141-147.

Henningfield JE and Fagerstrom KO. Swedish Match Company, Swedish snus and public health: a harm reduction experiment in progress? 2001. Tob Control 10(3): 253-257.

Hetherton J, Matheson A and Robson M. Recruitment by GPs during consultations in a primary care randomized controlled trial comparing computerized psychological therapy with clinical psychology and routine GP care: problems and possible solutions. 2004. Primary Health Care Research and Development 5(1): 5-10.

Hilberink SR, Jacobs JE, Bottema BJAM, de Vries H and Grol RPTM. Smoking cessation in patients with COPD in daily general practie (SMOCC): Six months' results. 2005. Prev Med 41(5-6): 822-827.

Hilberink SR, Jacobs JE, Schlösser M, Grol RPTM and de Vries H. Characteristics of patients with COPD in three motivational stages related to smoking cessation. 2006. Patient Educ Couns 61(3): 449-457.

Hill J. Cautions against the immoderate use of stuff the effects it must produce when this way taken into the body. 1761. R. Baldwin and J. Jackson, London. 
Holtrop JS, Wadland WC, Vansen S, Weismantel D and Fadel H. Recruiting health plan members receiving pharmacotherapy into smoking cessation counseling. 2005. Am J Manag Care 11(8): 501-507.

Hooten W, Wolter TD, Ames SC, Hurt RD, Vicjers KS, Offord KP and Hays J. Personality correlates related to tobacco abstinence following treatment. 2005. International Journal of Psychiatry in Medicine 35(1): 59-74.

Hosmer DW and Lemeshow S. Applied Logistic Regression. 1989. Wiley, New York.

Hoving C, Mudde AN and de Vries H. Intention to adopt a smoking cessation expert system within a self-selected sample of Dutch general practitioners. 2006a. Eur J Cancer Prev 15(1): 82-86.

Hoving C, Mudde AN and de Vries H. Effect of recruitment method and setting on the composition of samples consisting of adult smokers. 2007. Pat Ed Couns 65(1): 79-86.

Hoving EF, Mudde AN and de Vries H. Predictors of smoking relapse in a sample of Dutch adult smokers; the roles of gender and action plans. 2006b. Addict Behav 31(7): 1177-1189.

Hughes JR, Keely JP, Niaura R, Ossip-Klein DJ, Richmond RL and Swan GE. Measures of abstinence in clinical trials: issues and recommendations. 2003. Nic Tob Res 5(1): 13-25.

Hughes JR, Keely J and Naud S. Shape of the relapse curve and long-term abstinence among untreated smokers. 2004a. Addiction 99(1): 29-38.

Hughes JR, Stead LF and Lancaster T. Antidepressants for smoking cessation (review). 2004b. Cochrane Database Syst Rev (4): CD000031.

Hurt RD, Wolter TD, Rigotti N, Hays JT, Niaura R, Durcan MJ, Gonzales D, Sachs DPL, Johnston JA and Offord KP. Bupropion for pharmacologic relapse prevention to smoking. Predictors of outcome. 2002. Addictive Behaviors 27(4): 493-507.

Kandel DB and Chen K. Extent of smoking and nicotine dependence in the United States: 1991-1993. 2000. Nic Tob Res 2(3): 263-274. 
Kaper J, Wagena EJ, Severens JL and van Schayck CP. Healthcare financing systems for increasing the use of tobacco dependence treatment (review). 2005. Cochrane Database Syst Rev (1): CD004305.

Karwalajtys T, Kaczorowski J, Chambers LW, Levitt C, Dolovich L, McDonough B, Patterson C and Williams JE. A randomized trial of mail vs. telephone invitation to a community-based cardiovascular health awareness program for older family practice patients. 2005. BMC Fam Pract 6: 35.

Kenford SL, Smith SS, Wetter DW, Jorenby DE, Fiore MC and Baker TB. Predicting relapse back to smoking: contrasting affective and physical models of dependence. 2002. J Consult Clin Psychol 70(1): 216-227.

Kok G. Quality of planning as a decisive determinant of health education effectiveness. 1992. Hygie 11(4): 5-8.

Kok G, van den Borne B and Mullen PD. Effectiveness of health education and health promotion: meta-analyses of effect studies and determinants of effectiveness. 1997. Pat Ed Couns 30(1): 19-27.

Krall EA, Garvey AJ and Garcia RI. Smoking relapse after 2 years of abstinence: findings from the VA Normative Aging Study. 2002. Nicotine Tob Res 4(1): 95-100.

Kremers SPJ, Mudde AN and de Vries H. Subtypes within the precontemplation stage of adolescent smoking acquisition. 2001. Addictive Behaviors 26(2): 237-251.

Kreuter MW, Oswald DL, Bull FC and Clark EM. Are tailored health education materials always more effective than non-tailored materials? 2000. Health Educ Res 15(3): 305-315.

Lamkaddem M, de Bakker D, Nijland A and de Haan J. De invloed van praktijkondersteuning op de werklast van huisartsen. Een analyse van gegevens uit het Landelijk Informatie Netwerk Huisartsenzorg. 2004.

Lancaster T, Stead L, Silagy C and Sowden A. Effectiveness of interventions to help people stop smoking: findings from the Cochrane Library. 2000. BMJ 321(7257): 355-358.

Lancaster T and Stead LF. Self-help interventions for smoking cessation. 2005a. Cochrane Database of Systematic Reviews (3). 
Lancaster $\mathrm{T}$ and Stead LF. Individual behavioral counselling for smoking cessation. 2005b. Cochrane Database Syst Rev (2): CD001292.

Landelijke Huisartsen Vereniging. Over de LHV [About the LHV]. 2006. http://lhv.artsennet.nl/content/hp/

Larimer ME, Palmer RS and Marlatt GA. Relapse prevention. An overview of Marlatt's cognitive-behavioral model. 1999. Alcohol Res Health 23(2): 151-160.

Lee RE, McGinnis KA, Sallis JF, Castro CM, Chen AH and Hickmann SA. Active vs. passive methods of recruiting ethnic minority women to a health promotion program. 1997. Annals of Behavioral Medicine 19(4): 378-384.

Lloyd-Williams F. The effect of an intervention programme to improve health education leaflet uptake and distribution in community pharmacies. 2003. Patient Educ Couns 49(1): 27-33.

Lock CA, Kaner EFS, Heather N, Gilvarry E and McAvoy BR. Changes in receptionists' attitudes towards involvement in a general practice-based trial of screening and brief alcohol intervention. 2000. Br J Gen Pract 50(451): 111-115.

Locke EA and Latham GP. Building a practically useful theory of goal setting and task motivation. 2002. Am Psychol 57(9): 705-717.

Maguire TA, McElnay JC and Drummond A. A randomized controlled trial of a smoking cessation intervention based in community pharmacies. 2001. Addiction 96(2): 325-331.

Manchon Walsh P, Carrillo P, Flores G, Masuet C, Morchon S and Ramon JM. Effects of partner smoking status and gender on longterm abstinence rates of patients receiving smoking cessation treatment. 2007. Addict Behav 32(1): 128-136.

Margitic S, Sevick MA, Miller M, Albright C, Banton J, Callahan K, Garcia M, Gibbons L, Levine BJ and Anderson R. Challenges Faced in Recruiting Patients from Primary Care Practices into a Physical Activity Intervention Trial. 1999. Prev Med 29(4): 277-286.

Marlatt GA and Gordon JR. Relapse prevention: maintenance strategies in the treatment of addictive behaviors. 1985. Guilford Press, New York. 
Martinson BC, Lazovich D, Lando HA, Perry CL, McGovern PG and Boyle RG. Effectiveness of monetary incentives for recruiting adolescents to an intervention trial to reduce smoking. 2000. Prev Med 31(6): 706-713.

Matheny K and Weatherman K. Predictors of smoking cessation and maintenance. 1998. J Clin Psychol 54(2): 223-235.

McAvoy BR and Kaner EF. General practice postal surveys: a questionnaire too far? 1996. BMJ 313(7059): 732-3.

McAvoy BR, Kaner EF, Lock CA, Heather N and Gilvarry E. Our Healthier Nation: are general practitioners willing and able to deliver? A survey of attitudes to and involvement in health promotion and lifestyle counselling. 1999. Br J Gen Pract 49(440): 187-90.

McClure JB, Greene SM, Wiese C, Johnson KE, Alexander G and Strecher V. Interest in an online smoking cessation program and effective recruitment strategies: Results from Project Quit. 2006. Journal of Medical Internet Research 8(3): e14.

McDonald PW. Population-Based Recruitment for Quit-Smoking Programs: An Analytic Review of Communication Variables. 1999. Prev Med 28(6): 545-557.

McEwen A and West R. Smoking cessation activities by general practitioners and practice nurses. 2001. Tob Control 10(1): 27-32.

McIntosh S, Ossip-Klein DJ, Spada J and Burton K. Recruitment strategies and success in a multi-county smoking cessation study. 2000. Nic Tob Res 2(3): 281-284.

McVey D and Stapleton J. Can anti-smoking television advertising affect smoking behaviour? Controlled trial of the Health Education Authority for England's anti-smoking TV campaign. 2000. Tob Control 9(3): 273-282.

Milne S, Orbell S and Sheeran P. Combining motivational and volitional interventions to promote exercise participation: protection motivation theory and implementation intentions. 2002. Br J Health Psychol 7(2): 163-184.

Monso E, Campbell J, Tonnesen P, Gustavsson G and Morera J. Sociodemographic predictors of success in smoking intervention. 2001. Tob Control 10(2): 165-169.

Morrow M and Barraclough S. Tobacco control and gender in Southeast Asia. Part I: Malaysia and the Philippines. 2003. Health Promot Int 18(3): 255-264. 
Mudde AN and de Vries $\mathrm{H}$. The reach and effectiveness of a national mass media-led smoking cessation campaign in The Netherlands. 1999. Am J Public Health 89(3): 346-350.

Mumford EA, Levy DT, Gitchell JG and Blackman KO. Smokeless tobacco use 1992-2002: trends and measurement in the Current Population Survey-Tobacco Use Supplements. 2006. Tob Control 15(3): 166-171.

Nides MA, Rakos RF, Gonzales D, Murray RP, Tashkin DP, Bjornson Benson WM, Lindgren P and Connett JE. Predictors of initial smoking cessation and relapse through the first 2 years of the Lung Health Study. 1995. J Consult Clin Psychol 63(1): 60-69.

Nunnally JC. Psychometric Theory. 1978. McGraw-Hill, New York.

Ockene JK, Emmons KM, Mermelstein RJ, Perkins KA, Bonollo DS and Voorhees CC. Relapse and Maintenance Issues for Smoking Cessation. 2000. Health Psychol 19(1): 17-31.

Oenema A, Tan F and Brug J. Short-term efficacy of a web-based computer-tailored nutrition intervention: main effects and mediators. 2005. Ann Behav Med 29 (1):54-63.

Patrick DL, Cheadle A, Thompson DC, Diehr P, Koepsell T and Kinne S. The validity of self-reported smoking: a review and meta-analysis. 1994. Am J Public Health 84(7): 1086-1093.

Paulussen T, Kok G, Schaalma H and Parcel GS. Diffusion of AIDS curricula among Dutch secondary school teachers. 1995. Health Educ Q 22(2): 227-243.

Perkins KA. Smoking cessation in women: Special considerations. 2001. CNS Drugs 15(5): 391-411.

Prins GJJ and Willemsen MC. Tabakswetgeving in Nederland [Tobacco legislation in the Netherlands]. 2004. Hart Bulletin 35(4): 95-97.

Prochaska JO and DiClemente CC. Stages and processes of self-change of smoking: Toward an integrative model of change. 1983. J Consult Clin Psychol 51(3): 390395. 
Prochaska JO, Diclemente CC and Norcross JC. In search of how people change: Applications to addictive behaviors. 1992. Am Psychol 47(9): 1102-1114.

Prochaska JO, Redding CA and Evers KE. The Transtheoretical Model and Stages of Change. In: K Glanz. Health Behavior and Health Education: Theory, Research, and Practice. 1997. Jossey-Bass, San Francisco.

Prochaska JO and Velicer WF. Misinterpretations and misapplications of the transtheoretical model. 1997. Am J Health Promot 12(1): 11-12.

Pronk M, Blom L, Jonkers R and Bakker A. Community pharmacy and patient-oriented activities: the Dutch case. 2002. Patient Educ Couns 46(1): 39-45.

Pronk M, Blom A, Jonkers R, Van Burg A. The diffusion process of patient education in Dutch community pharmacy: an exploration. 2001. Patient Educ Couns 42(2):115-121.

Reubsaet A, Reinaerts EBM, Brug J, van Hooff JP and van den Borne HW. Process evaluation of a school-based education program about organ donation and registration, and the intention for continuance. 2004. Health Educ Res 19(6): 720-729.

Richards L. Using NVivo in Qualitative Research. 1999. QSR Pty Lmt, Buundora, Australia.

Rickards L, Fox K, Roberts C, Fletcher L and Goddard E. Living in Britain: results of the 2002 general household survey. 2004.

Rogers EM. Diffusion of Innovations. 1995. Free Press, New York.

Rogers EM. Diffusion of preventive innovations. 2002. Addict Behav 27(6): 989-993.

Rohrbaugh MJ, Shoham V, Trost S, Muramoto M, Cate RM and Leischow S. Couple dynamics of change-resistant smoking: Toward a family consultation model. 2001. Fam Proc 40(1): 15-31.

Ruggiero L, Webster K, Peipert JF and Wood C. Identification and recruitment of lowincome pregnant smokers: Who are we missing? 2003. Addict Behav 28(8): 1497-1505.

Ryan P and Lauver DR. The efficacy of tailored interventions. 2002. J Nurs Scholarsh 34(4): 331-337. 
Sanchez-Johnsen L, Ahluwalia JS, Fitzgibbon M and Spring BJ. Ethnic similarities and differences in reasons for smoking. 2006. Addict Behav 31(3): 544-548.

Sanderson Cox L, Patten CA, Niaura RS, Decker PA, Rigotti N, Sachs DPL, Buist A and Hurt RD. Efficacy of Bupropion for Relapse Prevention in Smokers With and Without a Past History of Major Depression. 2004. Journal of General Internal Medicine 19(8): 828-834.

Sarkin JA, Marshall SJ, Larson KA, Calfas KJ and Sallis JF. A Comparison of Methods of Recruitment to a Health Promotion Program for University Seniors. 1998. Prev Med 27(4): 562-571.

Scott Lennox A, Osman LM, Reiter E, Robertson R, Friend J, McCann I, Skatun D and Donnan PT. Cost effectiveness of computer tailored and non-tailored smoking cessation letters in general practice: randomised controlled trial. 2001. BMJ 322(7299): 1396.

Segaar D, Bolman C, Willemsen MC and De Vries H. Determinants of adoption of cognitive behavioral interventions in a hospital setting: Example of a minimalcontact smoking cessation intervention for cardiology wards. 2006. Patient Educ Couns 61(2): 262-271.

Senore C, Battista RN, Ponti A, Segnan N, Shapiro SH, Rosso S and Aimar D. Comparing participants and nonparticipants in a smoking cessation trial:selection factors associated with general practitioner recruitment activity. 1999. J Clin Epidemiol 52(1): 83-89.

Shiffman S, Paty JA, Rohay JM, Di Marino ME and Gitchell J. The Efficacy of ComputerTailored Smoking Cessation Material as a Supplement to Nicotine Polacrilex Gum Therapy. 2000. Arch Intern Med 160(11): 1675-1681.

Silagy C, Lancaster T, Stead L, Mant D and Fowler G. Nicotine replacement therapy for smoking cessation. 2004. Cochrane Database Syst Rev (3): CD000146.

Silverentand W. Kruideniers en kijkcijfers [Grocers and ratings]. 2003. Medisch Contact 58(8): 317-318. 
Sinclair HK, Bond CM, Lennox AS, Silcock J, Winfield AJ and Donnan PT. Training pharmacists and pharmacy assistants in the stage-of-change model of smoking cessation: a randomised controlled trial in Scotland. 1998. Tob Control 7(3): 253261.

Sinclair HK, Bond CM and Stead LF. Community pharmacy personnel interventions for smoking cessation (review). 2004. Cochrane Database Syst Rev (1): CD003698.

Skinner CS, Campbell MK, Rimer BK, Curry S and Prochaska JO. How effective is tailored print communication? 1999. Annals of Behavioral Medicine 21(4): 290298.

Slama K, Karsenty S and Hirsch A. French general practitioners' attitudes and reported practices in relation to their participation and effectiveness in a minimal smoking cessation programme for patients. 1999. Addiction 94(1): 125-132.

Smeets T, Brug J and de Vries H. Effects of tailoring health messages on physical activity. Health Educ Res (in press).

Sniehotta FF, Scholz U and Schwarzer R. Bridging the intention-behaviour gap: Planning, self-efficacy, and action control in the adoption and maintenance of physical exercise. 2005. Psychology and Health 20(2): 143-160.

Spittaels H, De Bourdeaudhuij I and Vandelanotte C. Evaluation of a web-delivered computer-tailored intervention for increasing physical activity in the general population. 2007. Prev Med 44(3): 209-217.

Stevens KR and Munoz LR. Cigarette smoking: Evidence to guide measurement. 2004. Research in Nursing \& Health 27(4): 281-292.

STIVORO for a smokefree future. Jaarverslag 1997 [Annual Report 1997]. 1998. http://www.stivoro.nl/actueel/jaarvers/jaarv97/index.html

STIVORO for a smokefree future. Jaarverslag 2001 [Annual Report 2001]. 2001a.

STIVORO for a smokefree future. Roken, de harde feiten 2001 [Smoking, the plain facts 2001]. 2001b.

STIVORO for a smokefree future. Evaluatie van activiteiten om het roken en meeroken bij de Nederlandse bevolking terug te dringen [Evaluation of activities to decrease smoking and passive smoking within the Dutch population]. 2003a. 
STIVORO for a smokefree future. 'Nederland start met stoppen' van start - Grootste stoppen met roken campagne ooit ['the Netherlands starts quitting' started Largest smoking cessation campaign ever]. 2003b. http://www.stivoro.nl/persberichten/200331022.html

STIVORO for a smokefree future. Stop effectief [Quit effectively]. 2006. http://www.stivoro.nl/hulpbijstoppen.html

Strecher VJ, Kreuter M, Den Boer DJ, Kobrin S, Hospers HJ and Skinner CS. The effect of computer-tailored smoking cessation messages in family practice settings. 1994. J Fam Pract 39(3): 262-270.

Strecher VJ. Computer-tailored smoking cessation materials: A review and discussion. 1999. Patient Educ Couns 36(2): 107-117.

Sugg Skinner C, Campbell MK, Rimer BK, Curry S and Prochaska JO. How effective is tailored print communication? 1999. Annals of Behavioral Medicine 21(4): 290298.

Sutton S. Back to the drawing board? A review of applications of the transtheoretical model to substance use. 2001. Addiction 96(1): 175-186.

Sutton S. Stage Theories of Health Behaviour. In: M Connor and P Norman. Predicting Health Behaviour: Research and Practice with Social Cognition Models. 2002. Open University Press, Buckingham.

Swan GE, Ward MM and Jack LM. Abstinence effects as predictors of 28-day relapse in smokers. 1996. Addictive Behaviors 21(4): 481-490.

Swartz LHG, Noell JW, Schroeder SW and Ary DV. A randomised control study of a fully automated internet based smoking cessation programme. 2006. Tob Contr 15(1): 7-12.

Tabachnick BG and Fidell LS. Using multivariate statistics. 2001. Pearson Education Company, Needham Heights, Massachusetts, USA.

Tomlin Z, Humphrey C and Rogers S. General practitioners' perceptions of effective health care. 1999. BMJ 318(7197): 1532-1535.

United States Public Health Service. Smoking and Health: Report of the Advisory Committee to the Surgeon General of the Public Health Service. 1964. 
USDHHS. Reducing the health concequences of smoking: 25 years of progress. A report of the Surgeon General. 1989.

Van den Berg M. De werkdruk van de huisarts [The GP's work-related pressure]. 2003. Medisch Contact 58(26/27): 1054-1056.

Van den Putte B, Yzer MC and Brunsting S. Social influences on smoking cessation: a comparison of the effect of six social influence variables. 2005. Prev Med 41(1): 186-193.

Velicer WF, DiClemente CC, Rossi JS and Prochaska JO. Relapse situations and selfefficacy: an integrative model. 1990. Addictive Behaviors 15(3): 271-283.

Velicer WF, Prochaska JO, Rossi JS and Snow MG. Assessing outcome in smoking cessation studies. 1992. Psychol Bull 111(1): 23-41.

Velicer WF, Norman GJ, Fava JL and Prochaska JO. Testing 40 predictions from the transtheoretical model. 1999. Addict Behav 24(4): 455-469.

Velicer WF and Prochaska JO. An expert system intervention for smoking cessation. 1999. Patient Educ Couns 36(2): 119-129.

Verplanken B and Faes S. Good intentions, bad habits, and effects of forming implementation intentions on healthy eating. 1999. European Journal of Social Psychology 29(5-6): 591-604.

Viswesvaran C and Schmidt FL. A meta-analytic comparison of the effectiveness of smoking cessation methods. 1992. Journal of Applied Psychology 77(4): 554-561.

Ward KD, Klesges RC and Halpern MT. Predictors of smoking cessation and state-ofthe-art smoking interventions. 1997. Journal of Social Issues 53(1): 129-145.

Weinstein ND. The precaution adoption process. 1988. Health Psychol 7(4): 355-386.

Weinstein ND, Rothman AJ and Sutton SR. Stage theories of health behavior: Conceptual and methodological issues. 1998. Health Psychol 17(3): 290-299.

Wensing M, Vedsted P, Kersnik J, Peersman W, Klingenberg A, Hearnshaw H, Hjortdahl P, Paulus D, Kunzi B, Mendive J and Grol R. Patient satisfaction with availability of general practice: an international comparison. 2002. Int J Qual Health Care 14(2): 111-118. 
West R, Hajek P, Stead L and Stapleton J. Outcome criteria in smoking cessation trials: proposal for a common standard. 2005. Addiction 100(3): 299-303.

Wetter DW, Kenford SL, Smith SS, Fiore MC, Jorenby DE and Baker TB. Gender differences in smoking cessation. 1999. J Consult Clin Psychol 67(4): 555-562.

Wiggers LCW, Smets EMA, Oort FJ, Storm-Versloot MN, Vermeulen H, van Loenen LBM, Peters RJG, de Haes HCJM and Legamate DA. Adherence to Nicotine Replacement patch Therapy in Cardiovascular patients. 2006. Int J Behav Med 13(1): 79-88.

Willemsen MC, Hoogenveen RT and van der Lucht F. New smokers and quitters; Transitions in smoking status in a national population. 2002. Eur J Public Health 12(2): 136-138.

Williams DM, Newsom JF and Brock TP. An evaluation of smoking cessation-related activities by pharmacists. 2000. J Am Pharm Assoc 40(3): 366-370.

Wynder EL, Bross IJ and Day E. A study of environmental factors in cancer of the larynx. 1956. Cancer 9(1): 86-110.

Yancey AK, Ortega AN and Kumanyika SK. Effective recruitment and retention of minority research participants. 2006. Annu Rev Public Health 27(8): 1-28.

Yarnall K, Pollak K, Krause K and Michener J. Primary care: Is there enough time for prevention? 2003. Am J Public Health 93(4): 635-641.

Zhu SH, Melcer T, Sun J, Rosbrook B and Pierce JP. Smoking cessation with and without assistance: A population-based analysis. 2000. Am J Prev Med 18(4): 305-311.

Zillich AJ, Aquilino ML and Farris KB. Knowledge and attitudes about smoking cessation among pharmacy technicians. 2004. J Am Pharm Assoc 44(5): 578-582. 



\section{Summary}

Tobacco smoking continues to be a major threat to health in the Netherlands; the percentage of smokers in the Netherlands (around 30\% of the Dutch population) has stabilized in the last years. Health care providers, such as general practitioners and pharmacists, have ample opportunities to engage smokers and provide them with cessation advice, which has shown to be effective. Nevertheless, many indicate that cessation advice is rarely given because of lack of time or skills. Computer tailoring can provide health care professionals with an opportunity to offer their smoking patients sound cessation advice without large time constraints for the professionals themselves.

The present thesis describes an effectiveness study regarding a computergenerated tailored smoking cessation expert system and its dissemination in Dutch general practice and community pharmacy. Health care staff was involved in the study by either actively (on staff's initiative) or passively (on smoker's initiative) offering the expert system to their smoking clients. Active recruitment was only conducted in community pharmacy.

In chapter 1, an overview is given regarding the extent of tobacco smoking, its consequences and the research that has been previously conducted to assess predictors of smoking behaviour and interventions effects. Furthermore, a description of the expert system and its theoretical framework, the I-Change Model, is provided. Based on the IPlan Model, six research questions were identified (see page 21-22).

Chapter 2 describes a cross-sectional study regarding the determinants of adoption of the expert system among GPs. A distinction was made between GPs were willing to adopt (adopters, $n=41$ ), unwilling to adopt (non-adopters, $n=35$ ) and GPs still in doubt (doubters, $\mathrm{n}=44$ ). Adopters had a more positive attitude the expert system, perceived more social influences in favour of giving smoking cessation advice and were more confident they would be able to use the expert system than non-adopters. Adopters expected less of a time investment compared to doubters. An important 
finding was that smoking cessation activities were mostly considered to be a task for the practice assistant.

In chapter 3 , the effectiveness of the expert system in both health care settings is tested in a randomized controlled design (control group did not receive any intervention) in two samples of adult smokers (545 smokers recruited in general practices, 474 in community pharmacies). A previous efficacy study had shown a significant effect of the expert system (providing a single tailored letter). However, results from the effectiveness study did not confirm these earlier findings; although the expert system significantly improved the number of cessation attempts in smokers recruited in community pharmacies twelve months after baseline, it did not increase abstinence rates.

The determinants of the intention to further implement the expert system after the end of the study was assessed in cross-sectional study described in chapter 4 . The sample included both GPs $(n=41)$ and general practice assistants $(n=48)$. Further implementation was determined by a social norm towards providing smoking cessation education and a higher self-efficacy. GPs were more likely to intend to implement the expert system than practice assistants.

Chapter 5 summarizes the findings from 33 telephone interviews with pharmacy assistants who had actively offered the expert system to their smoking clients. Assistants indicated that although smoking cessation is important, the pharmacy might not be the most suitable channel. Active recruitment was not implemented in a structural manner; busy periods, understaffing and negative responses from clients discouraged this.

Chapter 6 provides results from the comparison between recruitment strategies (active vs. passive) and settings (general practice vs. community pharmacy) used. In addition, the three samples recruited within a three-month period were compared to a cohort of Dutch smokers. Active recruitment resulted in more pre-contemplators than passive recruitment in the pharmacy setting. The sample recruited in general practice included more smokers with a lower educational level than passive recruitment in 
pharmacy setting. All three recruited samples included more women, heavier smokers and less pre-contemplators than the cohort sample.

In chapter 7, the predictive quality of the stage of change paradigm within the IChange Model was assessed by focusing on predictors of forward and backward stage transitions. Furthermore, the $\varnothing$ pattern and stage sequencing was assessed. Results replicated the $\varnothing$ pattern and most respondents transitioning forward transitioned to an adjacent stage. Forward transition from pre-contemplation was predicted by perceiving more pros of quitting. However, a higher self-efficacy was predictive of forward transitions from pre-contemplation, contemplation and preparation. Overall, limited support in favour of the stage of change paradigm was found.

Chapter 8 assessed predictors of relapse after attempting to quit for men and women separately. Overall, predictors of relapse were having more previous cessation attempts, undertaking a quit attempt without the aid of bupropion and being classified in a stage of change closer to action at baseline. For men, predictive of relapse were having a smoking partner and being in a stage closer to action at baseline. Women with a higher addiction level and those who felt less confident not to smoke when confronted with negative outcomes of cessation were more likely to relapse. The preference for the action plan to remove all smoking materials from the home was a borderline significant predictor of relapse for women and for the total sample.

Finally, chapter 9 summarizes and discusses the main findings from the studies presented in chapters 2-8. These findings are then converted into recommendations for future studies and practice. Finally, methodological issues regarding the effectiveness trial and the assessment of smoking behaviour, as well as practical issues regarding recruitment and drop-out are discussed. 



\section{Samenvatting}

Het roken van tabak blijft een grote bedreiging van de gezondheid in Nederland; al heeft het percentage rokers in Nederland (rond 30\% van de Nederlandse populatie) zich de afgelopen jaren gestabiliseerd. Zorgverleners, zoals huisartsen en apothekers, hebben volop de mogelijkheid om rokers op hun gedrag aan te spreken en hen van stopadvies te voorzien, waarvan welke eerdere studies de effectiviteit aangetoond hebben. Echter, veel van hen geven aan dit advies nauwelijks te verstrekken door een gebrek aan tijd of vaardigheden. Computer tailoring geeft zorgverleners de mogelijkheid hun patiënten te voorzien van een kwalitatief goed stopadvies zonder grote tijdsinvestering voor henzelf.

Dit proefschrift beschrijft een effectiviteitstudie van een computergegeneerde getailord stoppen met roken expert systeem onder volwassen rokers en de disseminatie van dit systeem in de Nederlandse huisartspraktijk en apotheek. Stafleden waren bij de studie betrokken door het actief (op het initiatief van de stafleden) dan wel passief (op het initiatief van de roker) aanbieden van het expert systeem aan rokende cliënten. Actieve rekrutering vond alleen plaats in de apotheek.

In hoofdstuk 1 wordt een overzicht gegeven van de omvang van de rookproblematiek, de consequenties verbonden aan het roken van tabak en het onderzoek wat eerder uitgevoerd is om voorspellers van rookgedrag en de effectiviteit van interventies te identificeren. Daarnaast wordt een beschrijving gegeven van het expert systeem en het theoretische kader hierachter; het I-Change Model. Ook worden zes onderzoeksvragen op basis van het I-Plan Model geformuleerd en gepresenteerd (zie pagina 21-22).

Hoofdstuk 2 beschrijft een cross-sectionele studie naar de determinanten van de adoptie van het expert systeem onder huisartsen. Er werd onderscheid gemaakt tussen huisartsen die tot adoptie wilden overgaan (adopters, $n=41$ ), die niet tot adoptie wilden overgaan (non-adopters, $n=35$ ) en huisartsen die nog twijfelden (twijfelaars, $n=44$ ). Adopters hadden een meer positieve attitude ten opzichte van het expert systeem, ondervonden een sociale norm welke meer gericht was op het geven van stoppen met roken advies en waren er meer zeker van dat ze het expert systeem zouden kunnen 
gebruiken dan non-adopters. Adopters verwachtten ook een minder grote tijdsinvestering veroorzaakt door de adoptie dan twijfelaars. Een belangrijke bevinding was dat huisartsen stoppen met roken activiteiten voornamelijk als een taak voor de assistent zagen.

In hoofdstuk 3 werd de effectiviteit van het expert systeem in beide gezondheidszorg settings getest in een gerandomiseerde studie (waarbij de controle groep geen interventie ontving) in twee steekproeven bestaande uit 1019 rokers (545 gerekruteerd in de huisartspraktijk, 474 in de apotheek). Een eerder uitgevoerde effectstudie liet een significant positief effect van het expert systeem zien (bestaande uit één getailorde brief). De resultaten van de effectiviteitstudie bevestigden de eerdere resultaten echter niet; hoewel het expert system significant tot meer stoppogingen na 12 maanden in de steekproef van rokers gerekruteerd in de apotheek leidde, verbeterde het de abstinentie niet.

De determinanten van de intentie om het expert systeem na het einde van de studie te implementeren werd in een cross-sectionele studie getoetst en beschreven in hoofdstuk 4. De steekproef omvatte zowel huisartsen $(n=41)$ als huisartsassistenten $(n=48)$. Verdere implementatie werd bepaald door een sociale norm om stoppen met roken activiteiten uit te voeren en een hogere eigen effectiviteit. Huisartsen waren eerder geneigd om het expert system te implementeren dan huisartsassistenten.

Hoofdstuk 5 vat de resultaten samen van 33 telefonische interviews met apothekersassistenten die het expert systeem actief aan rokende cliënten hadden aangeboden. De assistenten gaven aan dat hoewel ze stoppen met roken belangrijk vonden, de apotheek misschien niet het meest geschikte kanaal was. Actieve rekrutering werd niet structureel geïmplementeerd; drukke perioden, onderbezetting van personeel en negatieve reacties van cliënten verhinderden dit.

Hoofdstuk 6 verschaft resultaten van de vergelijking tussen de steekproeven van de gebruikte rekrutering strategieën (actief en passief) en settings (huisartspraktijk en apotheek). Daarnaast werden de drie steekproeven vergeleken met een Nederlands cohort van rokers. In de apotheek resulteerde actieve rekrutering in meer precontemplatoren dan passieve rekrutering. De steekproef gerekruteerd in 
huisartsenpraktijken omvatte meer rokers met een laag opleidingsniveau dan passieve rekrutering in de apotheek. Alle drie steekproeven bevatten significant meer vrouwen, zwaardere rokers en minder pre-contemplatoren dan het cohort.

In hoofdstuk 7 werd de voorspellende waarde van het stage of change paradigma binnen het I-Change Model beoordeeld door te focussen op voorspellers van voorwaartse en achterwaartse stage transities. Daarnaast werd er naar het $\varnothing$ patroon en stage volgorde gekeken. Resultaten repliceerden het $\varnothing$ patroon en de meeste respondenten die een voorwaartse transitie ondergingen, verplaatsen zich naar een opvolgende stage. Voorwaartse transitie vanuit pre-contemplatie werd voorspeld door het zien van meer voordelen van stoppen met roken. Echter, een hogere eigen effectiviteit voorspelde voorwaartse transitie van pre-contemplatie, contemplatie en preparatie. Over het algemeen werden er weinig resultaten in het voordeel van het stage of change paradigma gevonden.

Hoofdstuk 8 beoordeelde de voorspellers van terugval naar roken na een stoppoging voor mannen en vrouwen. Over het algemeen waren een groter aantal eerdere stoppogingen, een stoppoging zonder gebruik van bupropion and de classificatie in een stage of change in de richting van actie op de voormeting voorspellers van terugval. Voor mannen voorspelden het hebben van een rokende partner en de classificatie in een stage of change in de richting van actie op de voormeting terugval. Vrouwen met een hoger verslavingsniveau en minder eigen effectiviteit om niet te roken wanneer ze met negatieve uitkomsten van stoppen werd geconfronteerd waren eerder geneigd om terug te vallen. De voorkeur voor het actie plan om alle rookmaterialen uit huis te verwijderen was een randsignificante voorspeller van terugval voor vrouwen en de totale steekproef.

Tot slot vat hoofdstuk 9 de voornaamste bevindingen van de studie uit hoofdstuk 2-8 samen en bediscussieerd deze. Deze bevindingen worden dan verwerkt tot aanbevelingen voor vervolgonderzoek, evenals de praktijk. Bovendien worden methodologische aspecten betreffende de effectiviteitstudie, het meten van rookgedrag, de rekrutering van gezondheids professionals en drop-out bediscussieerd. 



\section{Dankwoord}

Het zit erop, het proefschrift is af! En hoewel alleen mijn naam op de voorkant staat, ben ik niet de enige die aan de totstandkoming dit proefschrift heeft bijgedragen. Ik wil dan ook graag een aantal mensen bedanken die de weg hier naar toe een stukje interessanter maakten.

Allereerst natuurlijk mijn promotoren Hein de Vries en Aart Mudde. Ik ben ontzettend blij dat jullie het met mij aangedurfd hebben, ook al waren er barrières zoals tijdsdruk en kennisachterstand. Fijn ook dat jullie het aantal beren dat ik op de weg zag flink hebben uitgedund. Hein, dank voor je vertrouwen in mij en je hulp op significante beslismomenten. Aart, je relativeringsvermogen was een lifesaver! Fijn dat ik altijd met vragen en frustraties bij je terecht kon.

Verder ben ik dank verschuldigd aan de leden van de leescommissie en de overige leden van de corona voor de tijd en moeite die zij in het lezen en beoordelen van dit proefschrift hebben gestoken.

Daarnaast wil ik ook graag mijn "voorganger" Monique Termote noemen, die het fundament heeft gelegd voor de uitvoering van een aantal studies. Ik vond samenwerken met jou een feestje. Ons eerste bezoek aan een deelnemende apotheek was meteen een reality check: niet iedereen was zo enthousiast over ons onderzoek als wij hadden gedacht. Toch hebben we het gefikst om 65 apotheken bereid te krijgen om deel te nemen en hen te trainen en te volgen; een niet geringe klus.

Ook hebben tijdens dit project een aantal assistenten de helpende hand geboden. Vooral Dorien Hodiamont heeft mij heel veel praktische beslommeringen uit handen kunnen nemen. Dorien, je hebt tijdens de huisartsenstudie veel werk verzet om huisartsen te werven, tevreden te houden en van projectmaterialen te voorzien. Het was denk ik voor ons beiden een drukke en leerzame maar bovenal gezellige tijd. Dank je wel voor je inzet. Ook de bijdragen van Lori Seraus, Jonathan van 't Riet en Karin van Poppelen hebben absoluut bijgedragen tot het 'boekje' wat u nu voor u ziet. 
Ook een woord van dank voor ZonMw, STIVORO voor een rookvrije toekomst (in het bijzonder Andree van Emst), MEMIC, het WinAP (Peter de Smet), het NHG (Ton Drenthen), de toenmalige DHVs Limburg en West-Brabant, de deelnemende apotheken en huisartsenpraktijken en natuurlijk de rokers die hun gegevens voor deze studie beschikbaar hebben gesteld.

Uiteraard wil ik alle (oud) collega's van de vakgroep Gezondheidsvoorlichting bedanken voor de gezellige tijd en praktische hulp die ik heb mogen ontvangen. In het bijzonder Liesbeth, mijn ex-kamergenootje, die waarschijnlijk meer gezeur van mij heeft moet aanhoren dan wie dan ook, op een kamer waar lekker kletsen met straf doorwerken gecombineerd kon worden. Fijn dat je mijn paranimf wilde zijn. Leon bedankt voor de computer ondersteuning en de broodnodige koffieservice om 's morgens op gang te komen, Marja en Josée voor het regelen van congresbezoek en de (financiële) beslommeringen rondom het project. Astrid, Marjan, Roos, Tamara, Evelien en dé Froukje (or the equivalent) voor de peptalks, statistische bijdragen, gezelligheid tijdens congresbezoeken en meer: eigenlijk alles wat ik op dat moment nodig had.

Natuurlijk mag ik familie en vrienden niet vergeten, dank voor jullie interesse en de broodnodige afleiding!

Pap en mam, jullie zijn gedurende het hele promotieproces (en ook zeker daarvoor) zeer nauw betrokken geweest bij alles waar ik mezelf instortte en altijd vol vertrouwen geweest over de afloop, een heel fijn gevoel. Dat jullie achter me staan is dan ook niet nieuw, hoewel het de eerste keer in de gedaante van paranimf is. Ik vind het geweldig dit met jullie te kunnen delen: ' $t$ wicht het ' $t$ red!

Lieve Derek, dank je voor je hulp en steun; het checken van het taalgebruik in mijn proefschrift heeft je een echt actieve rol binnen het promotieproces gegeven. Heerlijk, een in-house spelling en grammatica checker! Maar je bent nog zoveel meer, als ik het even niet meer zag zitten kon ik ook op je rekenen. Cheers dears!

Ciska 


\section{Curriculum Vitae}

Eefke Franciska Hoving was born in Groningen, The Netherlands, on the 15th of July 1977. After receiving her MAVO and HAVO diploma from the Freaylema Mavo in Slochteren and the Aletta Jacobs Scholengemeenschap in Hoogezand respectively, she started her bachelor study in Nutrition and Dietetics in 1995 at the Hanzehogeschool, Hogeschool van Groningen, graduating in 1999.

In the following year, she worked as a dietician in academic hospital, regional hospital and home care setting. She was also part of the editorial board for the Dutch Journal for Dieticians (Nederlands Tijdschrift voor Diëtisten).

In 2000, she started working as a research assistant at the department of Health Promotion and Health Education at the University of Maastricht. First on the project described in this thesis, later on similar projects on dietary and multiple health behavioural computer-generated tailoring interventions. In 2003, she returned to this project as a researcher and began working on the dissertation you now see before you. From 2005 on, she also worked on a post-ESFA project examining gender and cultural differences in adolescents for smoking uptake and cessation from six European countries.

The author is currently working as an assistant professor focusing on Health Communication in Health Care settings at the Department of Health Promotion and Health Education of the University of Maastricht. 


\section{Publication List}

\section{Papers from this thesis}

Hoving C, Mudde AN, de Vries H. (2006). Intention to adopt a smoking cessation expert system within a self-selected sample of Dutch general practitioners. Eur J Cancer Prev, 15(1), 82-86.

Hoving EF, Mudde AN, de Vries H. (2006). Predictors of smoking relapse in a sample of Dutch adult smokers; the roles of gender and action plans. Addict Behav, 31(7), 1177-1189.

Hoving EF, Mudde AN, de Vries H (2006). Smoking and the $\varnothing$ pattern; predictors of transitions through the Stages of Change. Health Educ Res, 21(3), 305-314.

Hoving C, Mudde AN, de Vries H (2007). Effect of recruitment method and setting on the composition of samples consisting of adult smokers. Patient Educ Couns, 65(1), 79-86.

Hoving C, Mudde AN, de Vries H (2007). Intention to implement a smoking cessation intervention in Dutch general practice. Health Education, 107(3), 307-315.

\section{Additional papers}

Hoving C, Reubsaet A, de Vries H (2007). Predictors of smoking stage transitions for adolescent boys and girls. Prev Med, 44(6), 485-489. 